\title{
Scattered Data Fitting by Direct Extension of Local Polynomials to Bivariate Splines
}

\author{
Oleg Davydov ${ }^{1)}$ and Frank Zeilfelder ${ }^{2)}$
}

\begin{abstract}
We present a new scattered data fitting method, where local approximating polynomials are directly extended to smooth $\left(C^{1}\right.$ or $\left.C^{2}\right)$ splines on a uniform triangulation $\Delta$ (the four directional mesh). The method is based on designing appropriate minimal determining sets consisting of whole triangles of domain points for a uniformly distributed subset of $\triangle$. This construction allows to use discrete polynomial least squares approximations to the local portions of the data directly as parts of the approximating spline. The remaining Bernstein-Bézier coefficients are efficiently computed by extension, i.e. using the smoothness conditions. To obtain high quality local polynomial approximations even for difficult point constellations (e.g. with voids, clusters, tracks), we adaptively choose the polynomial degrees by controlling the smallest singular value of the local collocation matrices. The computational complexity of the method grows linearly with the number of data points, which facilitates its application to large data sets. Numerical tests involving standard benchmarks as well as real world scattered data sets illustrate the approximation power of the method, its efficiency and ability to produce surfaces of high visual quality, to deal with noisy data, and to be used for surface compression.
\end{abstract}

\section{$\S 1$. Introduction}

Let $\Xi=\left\{\xi_{i}\right\}_{i=1}^{N} \subset \Omega$ be a finite set of arbitrarily distributed points in a domain $\Omega \subset \mathbb{R}^{2}$, with a real number $z_{i}$ assigned to each $\xi_{i}, i=1, \ldots, N$. The scattered data fitting problem is to find a (smooth) function $s$ defined on $\Omega$ that approximates these data, i.e. $s\left(\xi_{i}\right) \approx z_{i}, i=1, \ldots, N$. The quality of the approximation $s$ is not only measured in terms of the errors $\left|s\left(\xi_{i}\right)-z_{i}\right|$ at the data points. It is also expected that $s$ does not exhibit artificial oscillations, and, if the data come from a function $f$ defined on $\Omega$, i.e., $z_{i}=f\left(\xi_{i}\right), i=1, \ldots, N$, then $s$ approximates $f$ well everywhere on $\Omega$, as far as the information contained in the data allows this. If $f$ is smooth in $\Omega$ or in a subdomain of it, it is natural to require that $s$ does not have artificial discontinuities, ridges or other visual artifacts there, which is accomplished by imposing $C^{1}$ or, better, $C^{2}$ smoothness.

The scattered data fitting problem arises in numerous applied fields, for instance, in Geosciences, Medical Imaging, surface (re)construction in Computer

1) Mathematisches Institut, Justus-Liebig-Universität Giessen, D-35392 Giessen, Germany, oleg.davydov@math.uni-giessen.de

2) Universität Mannheim, Fakultät für Mathematik und Infomatik, Lehrstuhl Mathematik IV, 68131 Mannheim, Germany, zeilfeld@euklid.math.uni-mannheim.de 
Aided Geometric Design, Reverse Engineering, and Data Mining. The typical real world data sets are large (1,000,000 points or more) and therefore require fast and efficient fitting algorithms, i.e. algorithms which are ideally of linear complexity $\mathcal{O}(N)$ and provide high approximation power. Sometimes the approximation obtained from a large unevenly distributed data set has to be transmitted or stored for later use. In this case it becomes important that the number of its defining parameters is as small as possible, in particular much smaller than the number of parameters needed to store the data points (data compression or data reduction). Moreover, a scattered data method should perform well for noisy data since the data sets arising in applications are usually contaminated with measurement errors. Another important requirement is that the form of the approximation $s$ provided by a scattered data method should allow efficient processing, for example fast visualization of the surface. In particular, it is desirable that $s$ can be evaluated at any $M$ points with linear complexity $\mathcal{O}(M)$. These and other aspects of scattered data fitting are discussed in the vast literature on the subject, see the surveys in $[19,20,34,36,53,57]$.

The purpose of this paper is to present a new stable and efficient method for scattered data fitting capable of dealing with large and possibly noisy data sets with highly varying local density, with voids, clusters and tracks, leading to high quality artifact-free piecewise polynomial surfaces in triangle Bernstein-Bézier form.

By using a uniform four-directional mesh $\triangle$ covering $\Omega$, see Fig. 1 , we consider either the space $\mathcal{S}_{3}^{1}(\triangle)$ of $C^{1}$ piecewise cubics or certain subspaces of $\mathcal{S}_{6}^{2}(\triangle)$, i.e. $C^{2}$ splines of degree six. The basic idea of the method is to design for these spaces new minimal determining sets (MDS) with special features, see Fig. 3 and 5. Roughly speaking, these MDS consist of all domain points belonging to certain triangles that form a uniformly distributed subset $\mathcal{T}$ of $\Delta$. (The triangles in $\mathcal{T}$ are completely filled with black circles in Fig. 3 and 5.) As in the two-stage methods [58], we first determine polynomial approximations to the local portions of the data surrounding each triangle $T \in \mathcal{T}$. Note that we do not follow the approach of selecting a few points nearest to a center, as e.g. in [17]. Instead, we consider all points inside a circle covering $T$. (If the number of points in such a circle is accidentally very high, we perform a grid type local data thinning to save costs.) The local approximations are computed as least squares polynomials in Bernstein-Bézier form with respect to the corresponding triangles. The resulting approximating spline $s$ is obtained in a second stage using the smoothness conditions. Thus, the second stage in our approach amounts to an efficient and numerically stable procedure of extending the local polynomial approximations which are already pieces of the spline. In particular, we do not need to evaluate the dual basis functionals (e.g. function or derivative values at prescribed points) for the local polynomials, in contrast to the methods based on smooth finite elements.

Another key observation is that the degrees of local polynomial approximations have to be adjusted to the varying amounts of information contained in the local portions of data. Indeed, not only the local density of the data may vary, but also the local data may have hidden redundancies because of unfortunate distribution of 
points near some low order argebraic curves. As shown in [9], the norm of the least squares operator, and, as a consequence, the approximation power of corresponding local polynomial is directly affected by the minimal singular value $\left(\sigma_{\min }\right)$ of the local collocation matrix. Since reducing the degree of a polynomial increases the chance of a better conditioned collocation matrix, we use an automatic procedure to choose reasonable degrees: Computing the minimal singular value, we compare it with a prescribed tolerance and either accept the approximation if $\sigma_{\min }$ is not too small, or repeatedly reduce the degree. The fast and reliable computation of $\sigma_{\min }$ for $\mathcal{O}(N)$ small local collocation matrices is done by using well-established algorithms of numerical linear algebra. As a result, we obtain in our numerical examples surfaces of high approximation and visual quality without a need for the improvement of the shape by large scale optimization with respect to the degrees of freedom of the spline.

We show that our MDS leads to stable local bases for the spline spaces. The theoretical approximation order achievable with these bases is determined by the maximal degree $q$ of polynomial reproduction, where $q=3$ for the $C^{1}$ bases, and $q=5$ or $q=6$ for the two types of $C^{2}$ bases used below. The actual approximation order of the scattered data fitting method depends on the approximation power of the local polynomial approximations that in turn depends on the availability of sufficient information in each local subregion. Numerical tests of Section 6.2 show almost optimal approximation order of the method for random data. Note that the (local) approximation order is theoretically optimal in the subregions of sufficiently high data density, see Remark 5.4. In addition, motivated by symmetry considerations, we introduce averaged approximation operators that have the same theoretical approximation behaviour as their non-averaged counterparts, but have shown a better performance in our numerical tests.

The paper is organized as follows. In Section 2, we begin with some preliminaries on the spline spaces and Bernstein-Bézier techniques. Then, we describe our MDS, the approximation operators, and the adaptive algorithm of local discrete least squares approximation. The proofs of the properties of the MDS are given in Section 3. In Sections 4 and 5 we present the results on stable local bases and the approximation properties of the splines. Section 6 contains numerical examples. Here, we begin with some remarks on computational aspects. Then, we give tests on the approximation order, shape recovery, compression and denoising for some well known test functions. Finally, we present the tests with real world data, including a well known benchmark glacier data, a terrain data set with highly varying densities, and a raw multibeam echosounder data set of about 630,000 points with noise and outliers. The tests confirm the efficiency of the method and its ability to produce surfaces of high approximation and visual quality from virtually every type of scattered data.

\section{Data Fitting Method}

We give some preliminaries on spline spaces and describe the data fitting method. 


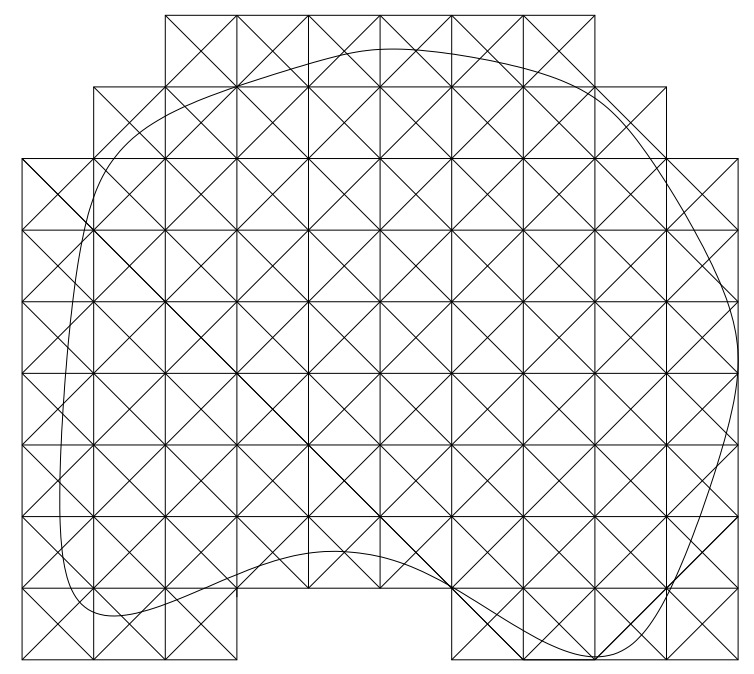

Fig. 1. The four directional mesh covering domain $\Omega$.

\subsection{Spline Spaces}

We start by briefly recalling well known notations related to the Bernstein-Bézier techniques for bivariate polynomial splines on triangulations (more details can be found e.g. in $[1,5-7,14,15,31])$.

Let $T:=\langle u, v, w\rangle \subset \mathbb{R}^{2}$ be a triangle. Given an integer $d$, let $B_{i j k}^{d}, i+j+k=d$, be the Bernstein polynomials of degree $d$ associated with $T$. It is well known that for every polynomial $p \in \mathcal{P}_{d}$ there is a unique Bernstein-Bézier representation

$$
p=\sum_{i+j+k=d} c_{i j k} B_{i j k}^{d} .
$$

We call the coefficients in the right hand side of (2.1) the BB-coefficients. Each BB-coefficient $c_{i j k}$ is associated with the domain point

$$
\eta_{i j k}^{T}:=(i u+j v+k w) / d .
$$

We denote the set of all domain points by

$$
\mathcal{D}_{d, T}:=\left\{\eta_{i j k}^{T}: i+j+k=d\right\} .
$$

Given a triangulation $\triangle$ covering a domain $\Omega \subset \mathbb{R}^{2}$, we denote by $\mathcal{S}_{d}^{r}(\triangle)$ the space of all $C^{r}$ piecewise polynomials with respect to $\triangle$,

$$
\mathcal{S}_{d}^{r}(\triangle):=\left\{s \in C^{r}(\Omega):\left.s\right|_{T} \in \mathcal{P}_{d} \text { for all triangles } T \in \triangle, T \cap \Omega \neq \emptyset\right\},
$$

where $\mathcal{P}_{d}$ is the space of bivariate polynomials of total degree $d$. It is well known that there is a one-one correspondence between the elements $s$ of the spline space $\mathcal{S}_{d}^{0}(\triangle)$ and the sequences of coefficients $c_{\eta}=c_{\eta}(s), \eta \in \mathcal{D}_{d, \triangle}$, with

$$
\mathcal{D}_{d, \triangle}:=\bigcup_{T \in \triangle} \mathcal{D}_{d, T}
$$


Here, for each $T \in \triangle$, and each $\eta=\eta_{i j k}^{T} \in T \cap \mathcal{D}_{d, \triangle}, c_{\eta}(s)$ is the coefficient $c_{i j k}$ in the representation (2.1) for $p=\left.s\right|_{T}$.

Let $\mathcal{S}$ be a linear subspace of $\mathcal{S}_{d}^{0}(\triangle)$. A set $\mathcal{M} \subseteq \mathcal{D}_{d, \triangle}$ is called a determining set for $\mathcal{S}$ if setting the coefficients of $s \in \mathcal{S}$ associated with the domain points in $\mathcal{M}$ to zero implies that all coefficients of $s$ corresponding to domain points in $\mathcal{D}_{d, \triangle}$ are zero. $\mathcal{M}$ is called a minimal determining set (MDS) for $\mathcal{S}$ if no proper subset $\mathcal{M}^{\prime} \subset \mathcal{M}$ is a determining set. Obviously, a determining set $\mathcal{M}$ is an MDS for $\mathcal{S}$ if and only if $\# \mathcal{M}=\operatorname{dim} \mathcal{S}$. (Throughout this paper, we denote the cardinality of a finite set $\mathcal{M}$ by $\# \mathcal{M}$.)

Of particular interest are the (BB-) smoothness conditions that allow us to express the smoothness of a spline in the form of some linear equations involving its BB-coefficients. Suppose $s$ is a spline in $\mathcal{S}_{d}^{0}(\triangle)$, and let $T:=\left\langle v_{1}, v_{2}, v_{3}\right\rangle$ and $\widetilde{T}:=\left\langle v_{4}, v_{3}, v_{2}\right\rangle$ be a pair of adjoining triangles in $\triangle$ sharing the edge $e=\left\langle v_{2}, v_{3}\right\rangle$. Let $c_{i j k}$ and $\tilde{c}_{i j k}$ be the BB-coefficients of $\left.s\right|_{T}$ and $\left.s\right|_{\widetilde{T}}$, respectively. Then $s$ is $C^{r}$ continuous across the edge $e$ if and only if

$$
\tilde{c}_{q, m-q, d-m}=\sum_{i+j+k=q} c_{i, j+d-m, k+m-q} B_{i j k}^{q}\left(v_{4}\right)
$$

for $m=q, \ldots, d$, and $q=1, \ldots, r$, where $B_{i j k}^{q}, i+j+k=q$, are the Bernstein polynomials of degree $q$ with respect to the triangle $T$ (cf. $[5,16]$ ).

Given $\Omega \subset \mathbb{R}^{2}$, we consider a uniform triangulation $\triangle$, called the four directional mesh, which covers $\Omega$, see Fig. 1 . For the sake of simplicity we give the details only for the square domain $\Omega=[0,1]^{2}$. Using $n+1$ vertical and horizontal lines we cover $\Omega$ with $n^{2}$ squares

$$
Q_{i, j}=\left[\frac{i-1}{n}, \frac{i}{n}\right] \times\left[\frac{n-j}{n}, \frac{n-j+1}{n}\right], \quad i, j=1, \ldots, n,
$$

and subdivide each of them into four subtriangles $T_{i, j}^{[k]}, k=1, \ldots, 4$, by inserting the two diagonals. (The triangles are numbered counterclockwise, starting from the leftmost subtriangle of $Q_{i, j}$.) In addition, throughout the theoretical part of the paper we assume that $n \geq 2$ is even.

In this paper we treat the cases $r=1$ and $r=2$ which are the most interesting for practical applications. Our approximation method is based on special MDS for the spaces $\mathcal{S}_{3}^{1}(\triangle)$ and $\mathcal{S}_{6}^{2}(\triangle)$ described below in Sections 2.2 and 2.3, respectively. We define our approximation operators in Section 2.4 and give the details of the adaptive algorithm for computing local least squares polynomial approximations in Section 2.5.

Remark 2.1. Note that the spaces $\mathcal{S}_{3}^{1}(\triangle)$ and $\mathcal{S}_{6}^{2}(\triangle)$ on the four directional mesh as well as more general quadrangulations with diagonals have been studied in a number of papers, see e.g. $[6,18,25-30,32,33,35,42-47,55]$. However, our approximation schemes are different from those known in the literature. 


$\begin{array}{lllll}{[0,6]} & {[2,6]} & {[4,6]} & {[6,6]} \\ {[1,5]} & {[3,5]} & {[5,5]} \\ {[0,4]} & {[2,4]} & {[4,4]} & {[6,4]} \\ {[1,3]} & {[3,3]} & {[5,3]} \\ {[0,2]} & {[2,2]} & {[4,2]} & {[6,2]} \\ {[1,1]} & {[3,1]} & {[5,1]} \\ {[0,0]} & {[2,0]} & {[4,0]} & {[6,0]}\end{array}$

Fig. 2. The indices $[m, \ell]$ of the domain points $\eta_{i, j}^{[m, \ell]}$ inside the square $Q_{i, j}\left(C^{1}\right.$ case).

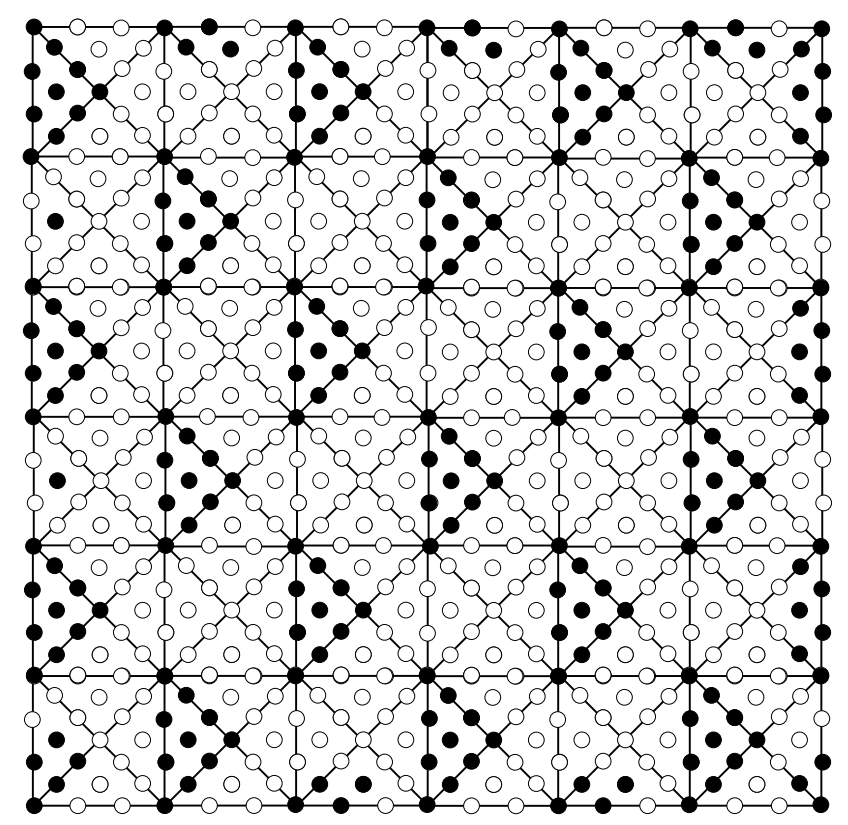

Fig. 3. The $\operatorname{MDS} \mathcal{M}$ for the $C^{1}$ spline space.

\subsection{MDS for $C^{1}$ Splines}

By using upper and lower bounds on the dimension of the spline spaces [59], we get

$$
\operatorname{dim} \mathcal{S}_{3}^{1}(\triangle)=5 n^{2}+8 n+3
$$

Because of the uniformity of the triangulation, the domain points exhibit a special uniform structure. See Fig. 3, where the domain points of $\mathcal{D}=\mathcal{D}_{3, \triangle}$ are shown as dots. Therefore, it will be convenient to use a special numeration of domain points. We set for $i, j=1, \ldots, n$,

$$
\mathcal{D} \cap Q_{i, j}=\left\{\eta_{i, j}^{[m, \ell]}:=\left(\frac{i-1}{n}+\frac{m}{6 n}, \frac{n-j}{n}+\frac{\ell}{6 n}\right): 0 \leq m, \ell \leq 6, m+\ell \text { even }\right\},
$$

and $c_{i, j}^{[m, \ell]}:=c_{\eta}$, if $\eta=\eta_{i, j}^{[m, \ell]}$ (see Fig. 2). 
We want to show that the set $\mathcal{M}$ of points marked by black dots in Fig. 3 is an MDS for $\mathcal{S}_{3}^{1}(\triangle)$. To describe it rigorously, we notice that the major part of $\mathcal{M}$ consists of $5 n^{2}$ points in

$$
\widetilde{\mathcal{M}}:=\mathcal{D} \cap \bigcup_{i+j \text { even }} T_{i, j}^{[1]}
$$

In addition, $\mathcal{O}(n)$ points are located near the boundary. More precisely, $\mathcal{M}$ is the union of $\widetilde{\mathcal{M}}$ with the sets

$$
\begin{aligned}
& \left\{\eta_{i, 1}^{[0,6]}, \eta_{i, 1}^{[1,5]}, \eta_{i, 1}^{[2,6]}, \eta_{i, 1}^{[3,5]}\right\}, \quad i \text { even, } \\
& \left\{\eta_{i, n}^{[0,0]}, \eta_{i, n}^{[1,1]}, \eta_{i, n}^{[2,0]}, \eta_{i, n}^{[3,1]}\right\}, \quad i \geq 3, \quad i \text { odd, } \\
& \left\{\eta_{1, j}^{[1,3]}\right\}, \quad j \text { even, } \\
& \left\{\eta_{1, n}^{[0,0]}, \eta_{1, n}^{[0,2]}, \eta_{1, n}^{[1,1]}, \eta_{1, n}^{[2,2]}\right\}, \\
& \left\{\eta_{n, j}^{[5,1]}, \eta_{n, j}^{[5,3]}, \eta_{n, j}^{[5,5]}, \eta_{n, j}^{[6,0]}, \eta_{n, j}^{[6,2]}, \eta_{n, j}^{[6,4]}, \eta_{n, j}^{[6,6]}\right\}, \quad j \text { odd, }, \quad \text { and } \\
& \left\{\eta_{n, n}^{[5,1]}, \eta_{n, n}^{[6,0]}, \eta_{n, n}^{[6,2]}\right\} .
\end{aligned}
$$

We will closely analyze below this particular $\operatorname{MDS} \mathcal{M}$, although the results are valid for any MDS for $\mathcal{S}_{3}^{1}(\triangle)$ containing $\widetilde{\mathcal{M}}$. Such a MDS may only differ from $\mathcal{M}$ by $\mathcal{O}(n)$ points on the boundary triangles.

Theorem 2.2. The set $\mathcal{M}$ is a minimal determining set for $\mathcal{S}_{3}^{1}(\triangle)$.

The proof of this theorem will be given in Section 3 .

\subsection{MDS for $C^{2}$ Splines}

Again by [59] we have

$$
\operatorname{dim} \mathcal{S}_{6}^{2}(\triangle)=19 n^{2}+24 n+6
$$

As before, the dots in Fig. 5 indicate the set $\mathcal{D}=\mathcal{D}_{6, \triangle}$ of domain points of the spline space. We set for $i, j=1, \ldots, n$,

$$
\mathcal{D} \cap Q_{i, j}=\left\{\eta_{i, j}^{[m, \ell]}:=\left(\frac{i-1}{n}+\frac{m}{12 n}, \frac{n-j}{n}+\frac{\ell}{12 n}\right): 0 \leq m, \ell \leq 12, m+\ell \text { even }\right\},
$$

and $c_{i, j}^{[m, \ell]}:=c_{\eta}$ if $\eta=\eta_{i, j}^{[m, \ell]}$ (see Fig. 4).

Let, furthermore, $\mathcal{M}^{*}$ be the set of points marked by black dots in Fig. 5 . We set

$$
\begin{gathered}
\widetilde{\mathcal{M}}=\mathcal{D} \cap \bigcup_{i+j \text { even }} T_{i, j}^{[1]}, \\
\mathcal{N}_{1}:=\bigcup_{i+j \text { even }}\left\{\eta_{i, j}^{[6,0]}, \eta_{i, j}^{[6,12]}, \eta_{i, j}^{[9,3]}, \eta_{i, j}^{[9,9]}, \eta_{i, j}^{[12,6]}\right\},
\end{gathered}
$$




$$
\begin{aligned}
& {[0,12][2,12] \quad[4,12] \quad[6,12] \quad[8,12] \quad[10,12] \quad[12,12]} \\
& {[1,11][3,11][5,11][7,11][9,11][11,11]} \\
& {[0,10][2,10][4,10][6,10][8,10][10,10][12,10]} \\
& {\left[\begin{array}{llllll}
{[1,9]} & {[3,9]} & {[5,9]} & {[7,9]} & {[9,9]} & {[11,9]}
\end{array}\right.} \\
& {\left[\begin{array}{llllllll}
{[0,8]} & {[2,8]} & {[4,8]} & {[6,8]} & {[8,8]} & {[10,8]} & {[12,8]}
\end{array}\right.} \\
& {[1,7] \quad[3,7] \quad[5,7] \quad[7,7] \quad[9,7] \quad[11,7]} \\
& {[0,6] \quad[2,6] \quad[4,6] \quad[6,6] \quad[8,6] \quad[10,6] \quad[12,6]} \\
& {[1,5] \quad[3,5] \quad[5,5] \quad[7,5] \quad[9,5] \quad[11,5]} \\
& {[0,4] \quad[2,4] \quad[4,4] \quad[6,4] \quad[8,4] \quad[10,4] \quad[12,4]} \\
& {[1,3] \quad[3,3] \quad[5,3] \quad[7,3] \quad[9,3] \quad[11,3]} \\
& {[0,2] \quad[2,2] \quad[4,2] \quad[6,2] \quad[8,2] \quad[10,2] \quad[12,2]} \\
& {[1,1] \quad[3,1] \quad[5,1] \quad[7,1] \quad[9,1] \quad[11,1]} \\
& {[0,0] \quad[2,0] \quad[4,0] \quad[6,0] \quad[8,0] \quad[10,0] \quad[12,0]}
\end{aligned}
$$

Fig. 4. The indices $[m, \ell]$ of the domain points $\eta_{i, j}^{[m, \ell]}$ inside the square $Q_{i, j}\left(C^{2}\right.$ case).

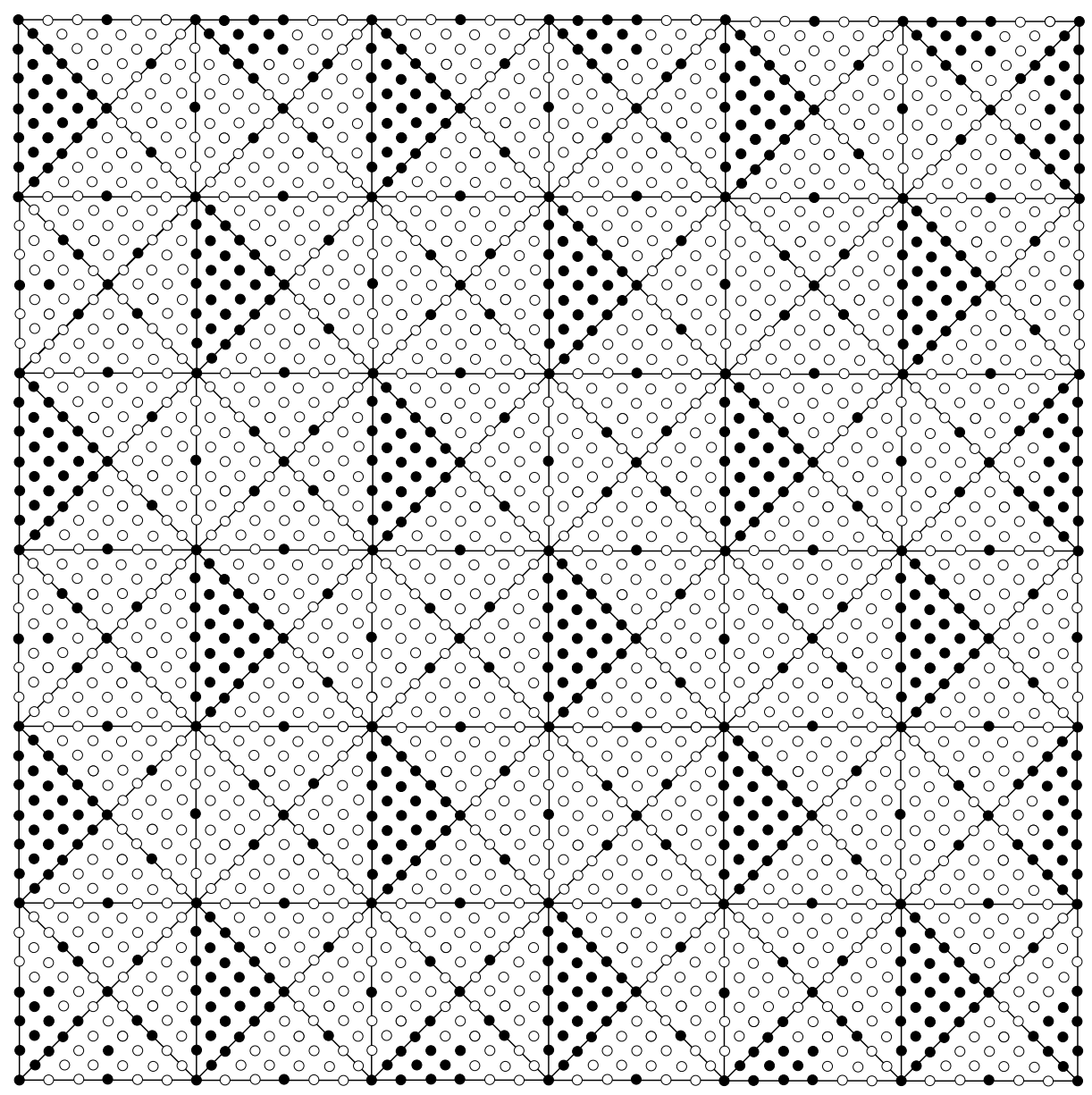

Fig. 5. The $\operatorname{MDS} \mathcal{M}$ for the $C^{2}$ spline space. 
and

$$
\mathcal{N}_{2}:=\bigcup_{i+j \text { odd }}\left\{\eta_{i, j}^{[4,4]}, \eta_{i, j}^{[4,8]}, \eta_{i, j}^{[6,6]} \eta_{i, j}^{[8,4]}, \eta_{i, j}^{[8,8]}\right\} .
$$

It is easy to see that $\# \widetilde{\mathcal{M}}=14 n^{2}$ and $\# \mathcal{N}_{1}=\# \mathcal{N}_{2}=5 n^{2} / 2$. To obtain $\mathcal{M}^{*}$, we add $\mathcal{O}(n)$ points near the boundary as in Fig. 5, such that $\mathcal{M}^{*}$ is the union of $\widetilde{\mathcal{M}} \cup \mathcal{N}_{1} \cup \mathcal{N}_{2}$ with the sets

$\left\{\eta_{i, 1}^{[0,12]}, \eta_{i, 1}^{[1,11]}, \eta_{i, 1}^{[2,10]}, \eta_{i, 1}^{[2,12]}, \eta_{i, 1}^{[3,9]}, \eta_{i, 1}^{[3,11]}, \eta_{i, 1}^{[4,10]}, \eta_{i, 1}^{[4,12]}, \eta_{i, 1}^{[5,11]}, \eta_{i, 1}^{[6,10]}, \eta_{i, 1}^{[9,9]}\right\}, i$ even $\left\{\eta_{i, n}^{[0,0]}, \eta_{i, n}^{[1,1]}, \eta_{i, n}^{[2,0]}, \eta_{i, n}^{[2,2]}, \eta_{i, n}^{[3,1]}, \eta_{i, n}^{[3,3]}, \eta_{i, n}^{[4,0]}, \eta_{i, n}^{[4,2]}, \eta_{i, n}^{[5,1]}, \eta_{i, n}^{[6,0]}, \eta_{i, n}^{[6,2]}, \eta_{i, n}^{[9,3]}\right\}$

$i \geq 3, i$ odd, $\left\{\eta_{1, j}^{[0,6]}, \eta_{1, j}^{[2,6]}, \eta_{1, j}^{[3,3]}, \eta_{1, j}^{[3,9]}\right\}, \quad j$ even $\left\{\eta_{1, n}^{[0,0]}, \eta_{1, n}^{[0,2]}, \eta_{1, n}^{[0,4]}, \eta_{1, n}^{[1,1]}, \eta_{1, n}^{[1,3]}, \eta_{1, n}^{[1,5]}, \eta_{1, n}^{[2,2]}, \eta_{1, n}^{[2,4]}, \eta_{1, n}^{[6,0]}, \eta_{1, n}^{[6,2]}, \eta_{1, n}^{[9,3]}\right\}$ $\left\{\eta_{n, j}^{[9,3]}, \eta_{n, j}^{[10,2]}, \eta_{n, j}^{[10,4]}, \eta_{n, j}^{[10,6]}, \eta_{n, j}^{[10,8]}, \eta_{n, j}^{[10,10]}, \eta_{n, j}^{[11,1]}, \eta_{n, j}^{[11,3]}, \eta_{n, j}^{[11,5]}, \eta_{n, j}^{[11,7]}, \eta_{n, j}^{[11,9]}\right.$ $\left.\eta_{n, j}^{[11,11]}, \eta_{n, j}^{[12,0]}, \eta_{n, j}^{[12,2]}, \eta_{n, j}^{[12,4]}, \eta_{n, j}^{[12,6]}, \eta_{n, j}^{[12,8]}, \eta_{n, j}^{[12,10]}, \eta_{n, j}^{[12,12]}\right\}, \quad j$ odd $\left\{\eta_{n, j}^{[9,9]}\right\}, \quad j \geq 3, \quad j$ odd, $\quad$ and $\left\{\eta_{n, n}^{[10,2]}, \eta_{n, n}^{[10,4]}, \eta_{n, n}^{[11,1]}, \eta_{n, n}^{[11,3]}, \eta_{n, n}^{[11,5]}, \eta_{n, n}^{[12,0]}, \eta_{n, n}^{[12,2]}, \eta_{n, n}^{[12,4]}\right\}$

As in the $C^{1}$ case, there exists modifications of the $\operatorname{MDS} \mathcal{M}^{*}$ for $\mathcal{S}_{6}^{2}(\triangle)$ which contain $\widetilde{\mathcal{M}} \cup \mathcal{N}_{1} \cup \mathcal{N}_{2}$. Such constructions differ only in the choice of points at the boundary, and the results remain valid.

Theorem 2.3. The set $\mathcal{M}^{*}$ is a minimal determining set for $\mathcal{S}_{6}^{2}(\triangle)$.

The proof of this theorem will be given in Section 3 .

Since we are interested in a MDS such that its major part only consists of points completely filling the triangles $T_{i, j}^{[1]}, i+j$ even, we remove the undesirable degrees of freedom (represented by points in $\mathcal{N}_{1} \cup \mathcal{N}_{2}$ ) by considering suitable subspaces of $\mathcal{S}_{6}^{2}(\triangle)$. This can be done by imposing certain super-smoothness and/or degree reduction conditions. We suggest two subspaces of this type, $\mathcal{S S}_{6}^{2}(\triangle)$ and $\mathcal{R S}_{6}^{2}(\triangle)$, as described below.

Let $\mathcal{S S}_{6}^{2}(\triangle)$ be the space of all splines $s \in \mathcal{S}_{6}^{2}(\triangle)$ with BB-coefficients $c_{i, j}^{[m, \ell]}=$ $c_{i, j}^{[m, \ell]}(s)$, which satisfy the following $C^{3}$ super-smoothness conditions:

$$
\begin{aligned}
8 c_{i, j}^{[0,12]} & =c_{i, j-1}^{[0,6]}+12 c_{i, j}^{[0,10]}-6 c_{i, j}^{[0,8]}+c_{i, j}^{[0,6]}, \quad \text { if } \quad i, j \geq 2, \\
8 c_{i, j}^{[3,3]} & =c_{i, j}^{[6,0]}+12 c_{i, j}^{[2,4]}-6 c_{i, j}^{[1,5]}+c_{i, j}^{[0,6]}, \\
8 c_{i, j}^{[3,9]} & =c_{i, j}^{[6,12]}+12 c_{i, j}^{[2,8]}-6 c_{i, j}^{[1,7]}+c_{i, j}^{[0,6]}, \\
8 c_{i, j}^{[6,6]} & =c_{i, j}^{[9,9]}+12 c_{i, j}^{[5,5]}-6 c_{i, j}^{[4,4]}+c_{i, j}^{[3,3]} \\
8 c_{i, j}^{[6,6]} & =c_{i, j}^{[9,3]}+12 c_{i, j}^{[5,7]}-6 c_{i, j}^{[4,8]}+c_{i, j}^{[3,9]}
\end{aligned}
$$


for all $i, j$ with $i+j$ even,

$$
\begin{aligned}
& 8 c_{i, n-1}^{[0,0]}=c_{i, n}^{[0,6]}+12 c_{i, n-1}^{[0,2]}-6 c_{i, n-1}^{[0,4]}+c_{i, n-1}^{[0,6]}, \quad i \geq 3, \quad i \text { odd }, \\
& 8 c_{n, j}^{[12,12]}=c_{n, j-1}^{[12,6]}+12 c_{n, j}^{[12,10]}-6 c_{n, j}^{[12,8]}+c_{n, j}^{[12,6]}, \quad j \geq 3, \quad j \text { odd }, \\
& 8 c_{n, n-1}^{[12,0]}=c_{n, n}^{[12,6]}+12 c_{n, n-1}^{[12,2]}-6 c_{n, n-1}^{[12,4]}+c_{n, n-1}^{[12,6]},
\end{aligned}
$$

and

(ii)

$$
\begin{aligned}
& 8 c_{i, j}^{[4,4]}=c_{i, j}^{[7,1]}+12 c_{i, j}^{[3,5]}-6 c_{i, j}^{[2,6]}+c_{i, j}^{[1,7]}, \\
& 8 c_{i, j}^{[4,8]}=c_{i, j}^{[7,11]}+12 c_{i, j}^{[3,7]}-6 c_{i, j}^{[2,6]}+c_{i, j}^{[1,5]}, \\
& 8 c_{i, j}^{[6,6]}=c_{i, j}^{[9,9]}+12 c_{i, j}^{[5,5]}-6 c_{i, j}^{[4,4]}+c_{i, j}^{[3,3]} \\
& 8 c_{i, j}^{[8,4]}=c_{i, j}^{[11,7]}+12 c_{i, j}^{[7,3]}-6 c_{i, j}^{[6,2]}+c_{i, j}^{[5,1]}, \\
& 8 c_{i, j}^{[8,8]}=c_{i, j}^{[11,5]}+12 c_{i, j}^{[7,9]}-6 c_{i, j}^{[6,10]}+c_{i, j}^{[5,11]},
\end{aligned}
$$

for all $i, j$ with $i+j$ odd.

Note that $s \in \mathcal{S S}_{6}^{2}(\triangle)$ is not necessarily $C^{3}$ at any point. Individual BernsteinBézier conditions of higher smoothness were used earlier in $[2,3,33]$ to define spline subspaces with desirable properties.

Another possibility to remove $\mathcal{N}_{1} \cup \mathcal{N}_{2}$ from the MDS is to reduce by one the polynomial degree of $\left.s\right|_{e}$ for certain edges $e$ of $\triangle$. Let $\mathcal{R S}_{6}^{2}(\triangle)$ be the space of all splines $s \in \mathcal{S}_{6}^{2}(\triangle)$ satisfying (2.10) and the following conditions which are an alternative to $(2.9)$ :

$$
\begin{aligned}
c_{i, j}^{[6,12]} & =\frac{1}{20}\left(c_{i, j}^{[0,12]}-6 c_{i, j}^{[2,12]}+15 c_{i, j}^{[4,12]}+15 c_{i, j}^{[8,12]}-6 c_{i, j}^{[10,12]}+c_{i, j}^{[12,12]}\right), \\
c_{i, j}^{[9,9]} & =\frac{1}{20}\left(c_{i, j}^{[6,6]}-6 c_{i, j}^{[7,7]}+15 c_{i, j}^{[8,8]}+15 c_{i, j}^{[10,10]}-6 c_{i, j}^{[11,11]}+c_{i, j}^{[12,12]}\right), \\
c_{i, j}^{[12,6]} & =\frac{1}{20}\left(c_{i, j}^{[12,0]}-6 c_{i, j}^{[12,2]}+15 c_{i, j}^{[12,4]}+15 c_{i, j}^{[12,8]}-6 c_{i, j}^{[12,10]}+c_{i, j}^{[12,12]}\right), \\
c_{i, j}^{[9,3]} & =\frac{1}{20}\left(c_{i, j}^{[6,6]}-6 c_{i, j}^{[7,5]}+15 c_{i, j}^{[8,4]}+15 c_{i, j}^{[10,2]}-6 c_{i, j}^{[11,1]}+c_{i, j}^{[12,0]}\right), \\
c_{i, j}^{[6,0]} & =\frac{1}{20}\left(c_{i, j}^{[0,0]}-6 c_{i, j}^{[2,0]}+15 c_{i, j}^{[4,0]}+15 c_{i, j}^{[8,0]}-6 c_{i, j}^{[10,0]}+c_{i, j}^{[12,0]}\right),
\end{aligned}
$$

for all $i, j$ with $i+j$ even. It is easy to see that (2.11) is equivalent to the requirement that $\left.s\right|_{e}$ is a polynomial of degree five for every edge $e$ of the triangles $T_{i, j}^{[k]}, k=$ $2,3,4, i+j$ even, which is not an edge of $T_{i, j}^{[1]}$.

Note that

$$
\mathcal{P}_{6} \subset \mathcal{S S}_{6}^{2}(\triangle), \quad \mathcal{P}_{5} \subset \mathcal{R S}_{6}^{2}(\triangle), \quad \mathcal{P}_{6} \not \subset \mathcal{R S}_{6}^{2}(\triangle)
$$


Therefore, $\mathcal{R S}_{6}^{2}(\triangle)$ has a reduced approximation power $\mathcal{O}\left(h^{6}\right)$ in contrast to the full approximation power $\mathcal{O}\left(h^{7}\right)$ of $\mathcal{S S}_{6}^{2}(\triangle)$, see Section 5 .

We set $\mathcal{M}=\mathcal{M}^{*} \backslash\left(\mathcal{N}_{1} \cup \mathcal{N}_{2}\right)$. As in the case of $C^{1}$ splines (see Section 2.1) the set $\mathcal{M}$ consists of the union of the points from $\widetilde{\mathcal{M}}$ in $(2.8)$ and the $\mathcal{O}(n)$ points included in $\mathcal{M}^{*}$ on the boundary.

Theorem 2.4. The set $\mathcal{M}$ is a minimal determining set for both $\mathcal{S S}_{6}^{2}(\triangle)$ and $\mathcal{R S}_{6}^{2}(\triangle)$. In particular,

$$
\operatorname{dim} \mathcal{S S}_{6}^{2}(\triangle)=\operatorname{dim} \mathcal{R S}_{6}^{2}(\triangle)=14 n^{2}+24 n+6
$$

The proof of this theorem will be given in Section 3 .

Remark 2.5. The above constructions are related to the investigations on Hermite and Lagrange interpolation with bivariate splines [10-13,41-52] which also employ the idea of decomposing the underlying triangulation and choosing different point constellations depending on the type of a triangle. Our MDS for $C^{1}$ splines is similar to the one suggested in [44] in the setting of so-called checkerboard triangulations. An essential difference is however that our MDS (away from the boundary) splits into groups completely filling certain triangles and leaving no extra degrees of freedom, thus facilitating the use of local polynomial approximations directly in Bernstein-Bézier form (see Section 2.4 and 2.5, below). If needed, this MDS may be transformed into a local Lagrange interpolation scheme, where the points of $\widetilde{\mathcal{M}}$ build the main part of the interpolation set. Moreover, we observe that, assuming that the boundary of the domain is treated appropriately, the above MDS in the $C^{2}$ case can also be easily transformed into a local Lagrange interpolation scheme for $\mathcal{S}_{6}^{2}(\triangle), \mathcal{S S}_{6}^{2}(\triangle)$ or $\mathcal{R S}_{6}^{2}(\triangle)$, respectively. This is possible due to the choice of points in the MDS, where certain triangles are completely filled, since in general there are no such simple one-to-one relation between the Bernstein-Bézier coefficients and (local) Lagrange interpolation points for smooth splines (cf. [46,49,52]). Other interpolation methods for bivariate splines use function and derivative values of the same form for every triangle or quadrilateral: these are the classical macro element methods $[8,18,54,55]$ and their recent extensions $[2,3,24-30,33]$. For applications of interpolation by bivariate splines to scattered data fitting, we refer the interested reader to the examples given in $[37,45,51,52]$. Further information on interpolation by bivariate splines can be found in the survey [49] and the references therein.

\subsection{Approximation Operators}

It is well-known (cf. [14]) that each element $\eta$ of an MDS $\mathcal{M}_{\mathcal{S}}$ for a bivariate spline space $\mathcal{S} \subset \mathcal{S}_{d}^{0}(\triangle)$ gives rise to a basis function $B_{\eta}$ for $\mathcal{S}$, where the spline $B_{\eta}$ is defined by setting all BB-coefficients in $\mathcal{M}_{\mathcal{S}}$ to zero, except for $c_{\eta}\left(B_{\eta}\right)=1$, and computing the remaining coefficients $c_{\xi}\left(B_{\eta}\right), \xi \in \mathcal{D}_{d, \triangle} \backslash \mathcal{M}_{\mathcal{S}}$, by using the 
smoothness conditions that define $\mathcal{S}$. Obviously, every spline $s \in \mathcal{S}$ can be written as

$$
s=\sum_{\eta \in \mathcal{M}_{\mathcal{S}}} c_{\eta}(s) B_{\eta}
$$

For constructing useful approximation operators for spline spaces $\mathcal{S}$ based on an MDS $\mathcal{M}_{\mathcal{S}}$, in many cases it is important that the splines $B_{\eta}, \eta \in \mathcal{M}_{\mathcal{S}}$, have local support. We recall that given a vertex $v$ of $\triangle, \operatorname{star}(v)=\operatorname{star}^{1}(v)$ is the union of triangles sharing $v$, and $\operatorname{star}^{\ell}(v), \ell \geq 2$, is defined recursively as the union of the stars of the vertices in $\operatorname{star}^{\ell-1}(v)$. A spline $s \in \mathcal{S}$ is called $\ell$-locally supported if there exists a vertex $v$ of $\triangle$ such that

$$
\operatorname{supp} s:=\overline{\{z \in \Omega: s(z) \neq 0\}} \subseteq \operatorname{star}^{\ell}(v) \text {. }
$$

In Section 4 we show that the basis splines $B_{\eta}$ for $\mathcal{S}_{3}^{1}(\triangle), \mathcal{S}_{6}^{2}(\triangle), \mathcal{S S}_{6}^{2}(\triangle)$ and $\mathcal{R S}_{6}^{2}(\triangle)$ corresponding to the MDS described in the previous subsections are 3locally supported.

Let $\mathcal{S}$ be one of the spaces $\mathcal{S}_{3}^{1}(\triangle), \mathcal{S S}_{6}^{2}(\triangle)$ or $\mathcal{R S}_{6}^{2}(\triangle)$, and $\mathcal{M}$ the respective MDS. We let $\widetilde{\mathcal{M}}$ be the set defined in Section 2.2 and 2.3 , respectively, set

$$
\widetilde{\mathcal{T}}:=\left\{T_{i, j}^{[1]}: \quad i+j \text { even }\right\}
$$

and split the set $\mathcal{M} \backslash \widetilde{\mathcal{M}}$ into disjoint subsets of points $\eta$ lying on the same boundary triangle $T \in \triangle \backslash \widetilde{\mathcal{T}}$. By adding these boundary triangles to $\widetilde{\mathcal{T}}$, we obtain a set of triangles in $\triangle$ denoted by $\mathcal{T}$. Let

$$
\mathcal{M}_{T}:=\mathcal{M} \cap T, \quad T \in \mathcal{T}
$$

For each $T \in \mathcal{T}$, let $p_{T}$ be a suitable polynomial approximation of a given function $f$ in a small subdomain $\Omega_{T}$ covering $T$. We define the approximation operator $Q$ by

$$
Q f=\sum_{T \in \mathcal{T}} \sum_{\eta \in \mathcal{M}_{T}} c_{\eta}\left(p_{T}\right) B_{\eta}
$$

Since $f$ is assumed to be known only at a discrete set of scattered points in

$$
\Xi=\left\{\xi_{i}: i=1, \ldots, N\right\} \subset \Omega
$$

we determine $p_{T}, T \in \mathcal{T}$, as polynomials whose values

$$
p_{T}(\xi), \quad \xi \in \Xi_{T}:=\Xi \cap \Omega_{T},
$$

approximate the corresponding values of $f$ in $\Xi_{T}$. This is done by applying the adaptive least squares algorithm described below in Section 2.5. 

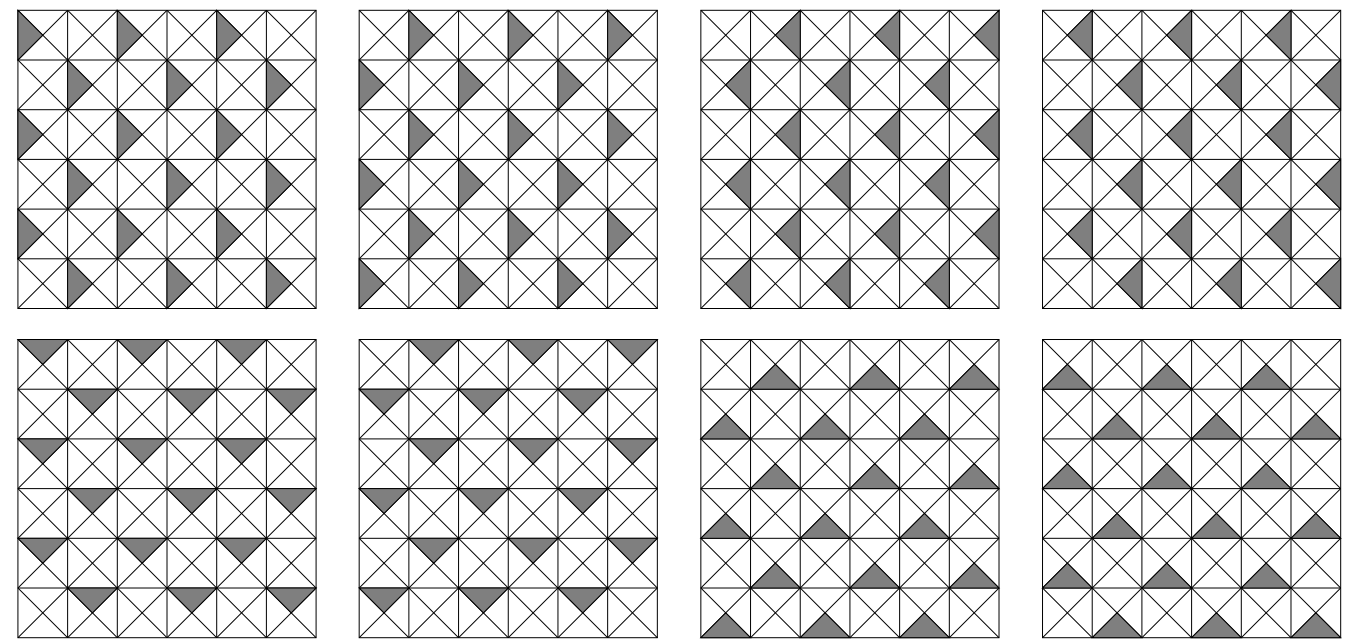

Fig. 6. Eight patterns of the set $\widetilde{\mathcal{T}}$.

Since there is some arbitrariness in the choice of the subset $\widetilde{\mathcal{T}}$ of $\triangle$ related to the position of the coordinate system, we also consider the average

$$
Q^{a v} f:=\frac{1}{8} \sum_{i=1}^{8} Q^{[i]} f
$$

of the eight operators $Q^{[i]}, i=1, \ldots, 8$, of type (2.13) corresponding to the eight possible patterns of the set $\widetilde{\mathcal{T}}$ shown in Fig. 6. Note that the computation of $Q^{a v} f \in \mathcal{S}$ involves local polynomial approximations $p_{T}$ defined for all $T \in \triangle$ since the eight sets $\mathcal{T}^{[i]}$ corresponding to $Q^{[i]}, i=1, \ldots, 8$, completely cover $\triangle$. According to our tests (see the numerical examples in Section 6) the operator $Q^{a v} f$ usually gives better results than $Q f$ at the expense of (about eight times) higher computational costs.

The approximation power of the above operators is studied in Section 5 .

Remark 2.6. Although it is not obvious how to generalize our MDS to general quadrangulations (cf. $[45,46]$ ), the $C^{1}$ scheme extends in a straight forward manner to the "deformed" four directional meshes, i.e. triangulations obtained by adding both diagonals to every quadrilateral of a strictly convex quadrangulation whose interior vertices are of degree four. (This is the particular case of checkerboard triangulations considered in detail in [44].) The averaged operator can also be defined for these triangulations.

\subsection{Adaptive Local Discrete Least Squares Polynomials}

Let $d=3$ in the $C^{1}$ case and $d=6$ in the $C^{2}$ case. For convenience, we assume that the data $z_{i}$ are the values of a function $f: \Omega \rightarrow \mathbb{R}$, i.e. $z_{i}=f\left(\xi_{i}\right), i=1, \ldots, N$. As pointed out in Section 2.4, we determine a local approximation polynomial $p_{T} \in \mathcal{P}_{d}$ 
for each triangle $T \in \mathcal{T}$, by using the data values $f(\xi), \xi \in \Xi_{T}=\Xi \cap \Omega_{T}$, in a suitable subdomain $\Omega_{T} \subset \Omega$ surrounding $T$. This is done by computing the (BB-) coefficients $c_{i j k}^{d, T}$ of $p_{T}$ in the Bernstein-Bézier form

$$
p_{T}=\sum_{i+j+k=d} c_{i j k}^{d, T} B_{i j k}^{d}
$$

where $B_{i j k}^{d}, i+j+k=d$, are the Bernstein polynomials of degree $d$ associated with $T$, as a solution of the discrete least squares problem: determine $c_{i j k}$ such that the error

$$
\sum_{\xi \in \Xi_{T}}\left(\sum_{i+j+k=d} c_{i j k} B_{i j k}^{d}(\xi)-f(\xi)\right)^{2}
$$

is minimized. As the most reliable method for solving this problem, we use the singular value decomposition of the $\left(\# \Xi_{T}\right) \times\left(\begin{array}{c}d+2 \\ 2\end{array}\right)$ matrix

$$
M_{d, T}:=\left[B_{i j k}^{d}(\xi)\right]_{i+j+k=d, \xi \in \Xi_{T}} .
$$

Thus, the coefficient vector $\left(c_{i j k}^{d, T}\right)_{i+j+k=d}$ is computed as the product of the pseudoinverse $M_{d, T}^{+}$of $M_{d, T}$ with the vector $(f(\xi))_{\xi \in \Xi_{T}}$ (cf. [4]). However, we accept the resulting polynomial $p_{T}$ as a reliable approximation of the local data only if the matrix $M_{d, T}$ has full rank and the reciprocal of its minimal singular value $\sigma_{d, T}$ does not exceed a tolerance value $\kappa$,

$$
\sigma_{d, T}^{-1} \leq \kappa
$$

The value of $\kappa$ is an important parameter of our method as it can be seen from the numerical results and discussion in Section 6.

If (2.17) fails, we conclude that the local distribution of data points does not permit a stable approximation with polynomials of degree $d$. In this situation we reduce the degree by one, and compute in the same way a least squares polynomial $p_{T}$ of degree $d-1$ in the form

$$
p_{T}=\sum_{i+j+k=d-1} c_{i j k}^{d-1, T} B_{i j k}^{d-1}
$$

where $B_{i j k}^{d-1}$ are the Bernstein polynomials of degree $d-1$ associated with $T$. If needed, we repeat the process and further reduce the degree of $p_{T}$ to $q=d-2, d-$ $3, \ldots, 0$. This algorithm of degree reduction terminates at degree $q>0$ as soon as the minimal singular value $\sigma_{q, T}$ of the matrix $M_{q, T}=\left[B_{i j k}^{q}(\xi)\right]_{i+j+k=q, \xi \in \Xi_{T}}$ satisfies $\sigma_{q, T}^{-1} \leq \kappa$. In this case the local approximating polynomial $p_{T}$ will be of degree $q$. If $\sigma_{q, T}^{-1}>\kappa$ for all $q=d, d-1, \ldots, 1$, we compute $p_{T}$ as the best least squares constant approximation to $f(\xi), \xi \in \Xi_{T}$, which is uniquely solvable 
as soon as $\# \Xi_{T} \geq 1$. Hence, this procedure of adjusting the degree of the local approximation polynomials is numerically stable.

If the degree of $p_{T}$ is reduced to some $q<d$, the representation (2.15) of $p$ with respect to Bernstein-Bézier polynomials of degree $d$ can be efficiently computed by well-known degree raising formulas, see [16].

Note that it is also possible to start the above procedure with some degree $q_{0}<d$ instead of $d$. This seems reasonable, for example, for the approximation operators based on the space $\mathcal{R S}_{6}^{2}(\triangle)$ which does not contain all polynomials of degree $d$. Indeed, we use $q_{0}=5$ in this case, see Section 5 .

Remark 2.7. The adaptive treatment of the local data is necessary for the computation of appropriate local polynomial approximants as soon as the points are allowed to be arbitrarily distributed. Detailed justification of the above procedure of local polynomial approximation can be found in [9]. Computational aspects of this adaptive approach are also discussed in Section 6.1.

Remark 2.8. Since we compute the least squares polynomial $p_{T}$ directly in its Bernstein-Bézier form, the coefficients $c_{\eta}\left(p_{T}\right), \eta \in \mathcal{M}_{T}, T \in \mathcal{T}$, of the approximating spline $Q f$ needed in (2.13) are immediately available. In particular, we have $\left.Q f\right|_{T}=p_{T}, T \in \mathcal{T}$. Thus, we avoid any intermediate estimates of the values of $f$ or its derivatives. The remaining coefficients of $Q f$ in its Bernstein-Bézier representation can than be computed step by step using the smoothness conditions (2.4) (see the particular form (3.1)-(3.2), respectively (3.3)-(3.5), they take for $r=1$ and $r=2$, respectively), where in the $C^{2}$ case the additional conditions (2.9) or (2.11), and (2.10) are used. (Concerning the treatment of the boundary we refer to the discussion in Section 6.1.) Therefore, the computation of the approximating spline does not require a (pre)computation of the basis splines $B_{\eta}$ in (2.13), which are, on the other hand, a useful tool to analyze the approximation properties of the method (see Sections 4 and 5).

Remark 2.9. Our method does not employ the minimization of the global least squares error or other global non-linear functionals such as the surface energy, thus avoiding computational expenses needed to solve such a large scale optimization problem. Nevertheless, the numerical examples in Section 6 show that our localized method is able to produce smooth artifact-free spline surfaces from difficult scattered data.

Remark 2.10. The adaptivity of the local stage can certainly be increased by designing appropriate algorithms of choosing the tolerance $\kappa$ individually for each triangle $T \in \mathcal{T}$, or by using the best discrete $\ell_{1}$ or $\ell_{\infty}$ polynomials where they are more appropriate. In addition, algorithms that take into account the anisotropies and statistical properties of the local data can be also thought of.

\section{§3. Minimal Determining Sets}

In this section, we show that the sets constructed in Sections 2.2 and 2.3 are minimal determing sets of the corresponding spline spaces, i.e., we prove Theorems 2.2, 2.3, 
and 2.4. Throughout this section we use the notations introduced in Section 2.2 and 2.3 , respectively.

Proof of Theorem 2.2: It is easy to see that $\# \mathcal{M}=5 n^{2}+8 n+3$, which coincides with the dimension of $\mathcal{S}_{3}^{1}(\triangle)$ in view of $(2.5)$. Therefore, it suffices to show that $\mathcal{M}$ is a determining set. To this end, given $s \in \mathcal{S}_{3}^{1}(\triangle)$, we suppose that

$$
c_{\eta}:=c_{\eta}(s)=0, \quad \text { all } \eta \in \mathcal{M} \text {. }
$$

We have to show that $c_{\eta}=0$ for all $\eta \in \mathcal{D}$. To do this, we use the smoothness conditions (2.4) of order 1 necessarily satisfied by $s$. These include

$$
\begin{aligned}
2 c_{i, j}^{[m, m]} & =c_{i, j}^{[m+1, m-1]}+c_{i, j}^{[m-1, m+1]}, \quad m=1, \ldots, 5, \\
2 c_{i, j}^{[m, 6-m]} & =c_{i, j}^{[m+1,6-m+1]}+c_{i, j}^{[m-1,6-m-1]}, \quad m=1, \ldots, 5,
\end{aligned}
$$

to ensure the $C^{1}$-smoothness of $s$ across the two diagonals of each $Q_{i, j}, i, j=$ $1, \ldots, n$, and

$$
\begin{aligned}
c_{i, j}^{[6, m]} & =c_{i+1, j}^{[0, m]}, m=0,2,4,6, i=1, \ldots, n-1, j=1, \ldots, n, \\
c_{i, j}^{[5, m]}+c_{i+1, j}^{[1, m]} & =c_{i, j}^{[6, m+1]}+c_{i, j}^{[6, m-1]}, m=1,3,5, i=1, \ldots, n-1, j=1, \ldots, n, \\
c_{i, j}^{[m, 0]} & =c_{i, j+1}^{[m, 6]}, m=0,2,4,6, i=1, \ldots, n, j=1, \ldots, n-1, \\
c_{i, j}^{[m, 1]}+c_{i, j+1}^{[m, 5]} & =c_{i, j}^{[m+1,0]}+c_{i, j}^{[m-1,0]}, m=1,3,5, i=1, \ldots, n, j=1, \ldots, n-1,
\end{aligned}
$$

for the $C^{1}$-smoothness of $s$ across interior vertical and horizontal sides of the squares. In the following, we only consider the "interior" part of $\mathcal{D}$, namely, $\eta \in \mathcal{D} \cap Q_{i, j}$, where $i, j \in\{2, \ldots, n-1\}$. The proof that $c_{\eta}=0$ for the remaining $\eta \in \mathcal{D}$ follows along the same lines, with an appropriate use of the points in $\mathcal{M} \backslash \overline{\mathcal{M}}$.

We consider two cases.

Case 1: $i+j$ even. First, it is clear that $c_{\eta}=0$ for all $\eta \in \mathcal{D} \cap T_{i, j}^{[1]}$. Obviously, there are only five points $\eta$ in $\mathcal{D} \cap T_{i, j}^{[2]}$ that are not included in $\mathcal{D} \cap\left(T_{i, j}^{[1]} \cup T_{i+1, j+1}^{[1]}\right)$, namely

$$
\eta \in\left\{\eta_{i, j}^{[2,0]}, \eta_{i, j}^{[3,1]}, \eta_{i, j}^{[4,0]}, \eta_{i, j}^{[4,2]}, \eta_{i, j}^{[5,1]}\right\}
$$

By (3.1), we have

$$
c_{i, j}^{[m, m-2]}=2 c_{i, j}^{[m-1, m-1]}-c_{i, j}^{[m-2, m]}=0, \quad m=2,3,4
$$

Using (3.2) we get

$$
c_{i, j+1}^{[5,5]}=c_{i+1, j+1}^{[0,4]}+c_{i+1, j+1}^{[0,6]}-c_{i+1, j+1}^{[1,5]}=0,
$$


and therefore from (3.1) and (3.2) we obtain successively

$$
\begin{aligned}
& c_{i, j}^{[4,0]}=2 c_{i, j+1}^{[5,5]}-c_{i+1, j+1}^{[0,4]}=0, \\
& c_{i, j}^{[5,1]}=c_{i, j}^{[4,0]}+c_{i+1, j+1}^{[0,6]}-c_{i, j+1}^{[5,5]}=0 .
\end{aligned}
$$

It follows that $c_{\eta}=0$ for all $\eta \in \mathcal{D} \cap T_{i, j}^{[2]}$. Similarly, $c_{\eta}=0$ for all $\eta \in \mathcal{D} \cap T_{i, j}^{[4]}$. Finally, (3.1) implies that $c_{\eta}=0$ for $\eta \in\left\{\eta_{i, j}^{[5,3]}, \eta_{i, j}^{[6,2]}, \eta_{i, j}^{[6,4]}\right\}$, and hence $c_{\eta}=0$ for all $\eta \in \mathcal{D} \cap Q_{i, j}, i+j$ even.

Case 2: $i+j$ odd. Case 1 implies that that $c_{\eta}=0$ for all

$$
\eta \in \mathcal{D} \cap\left(T_{i+1, j}^{[1]} \cup T_{i, j-1}^{[2]} \cup T_{i-1, j}^{[3]} \cup T_{i, j+1}^{[4]}\right)
$$

Hence, it follows from $(3.1)$ (for $m \in\{1,5\}$ ) that $c_{\eta}=0$ for all

$$
\eta \in\left\{\eta_{i, j}^{[1,1]}, \eta_{i, j}^{[1,5]}, \eta_{i, j}^{[5,1]}, \eta_{i, j}^{[5,5]}\right\}
$$

Moreover, by (3.2) (for $m=3$ ), we have $c_{\eta}=0$ for all

$$
\eta \in\left\{\eta_{i, j}^{[1,3]}, \eta_{i, j}^{[3,1]}, \eta_{i, j}^{[3,5]}, \eta_{i, j}^{[5,3]}\right\}
$$

Hence, (3.1) (for $m \in\{2,3,4\}$ ) implies that $c_{\eta}=0$ for the remaining $\eta \in \mathcal{D} \cap Q_{i, j}$.

Proof of Theorem 2.3: Since $\# \mathcal{M}^{*}=19 n^{2}+24 n+6=\operatorname{dim} \mathcal{S}_{6}^{2}(\triangle)$, which is easy to check by inspection, it suffices to show that $\mathcal{M}^{*}$ is a determining set. To this end, given $s \in \mathcal{S}_{6}^{2}(\triangle)$, we suppose that

$$
c_{\eta}:=c_{\eta}(s)=0, \quad \text { all } \eta \in \mathcal{M}^{*} .
$$

We have to show that then $c_{\eta}=0$ for all $\eta \in \mathcal{D}$. As in the proof of Theorem 2.2, we start by reformulating the smoothness conditions (2.4) satisfied by $s$ in the appropriate notation introduced in Section 2.3. The $C^{2}$-smoothness of $s$ across the two diagonals of each $Q_{i, j}, i, j=1, \ldots, n$, leads to the equations

$$
\begin{aligned}
2 c_{i, j}^{[m, m]} & =c_{i, j}^{[m+1, m-1]}+c_{i, j}^{[m-1, m+1]}, \quad m=1, \ldots, 11, \\
4 c_{i, j}^{[m, m]} & =c_{i, j}^{[m+2, m-2]}+4 c_{i, j}^{[m-1, m+1]}-c_{i, j}^{[m-2, m+2]}, \quad m=2, \ldots, 10, \\
2 c_{i, j}^{[m, 12-m]} & =c_{i, j}^{[m+1,12-m+1]}+c_{i, j}^{[m-1,12-m-1]}, \quad m=1, \ldots, 11 \\
4 c_{i, j}^{[m, 12-m]} & =c_{i, j}^{[m+2,12-m+2]}+4 c_{i, j}^{[m-1,12-m-1]}-c_{i, j}^{[m-2,12-m-2]}, \quad m=2, \ldots, 10 .
\end{aligned}
$$


The $C^{2}$-smoothness across interior vertical sides of the squares for $i=1, \ldots, n-$ $1, j=1, \ldots, n$, is given by

$$
\begin{aligned}
c_{i, j}^{[12, m]}= & c_{i+1, j}^{[0, m]}, \quad m=0,2,4,6,8,10,12, \\
c_{i, j}^{[11, m]}+c_{i+1, j}^{[1, m]}= & c_{i, j}^{[12, m+1]}+c_{i, j}^{[12, m-1]}, \quad m=1,3,5,7,9,11, \\
c_{i+1, j}^{[2, m]}= & c_{i, j}^{[12, m-2]}+c_{i, j}^{[12, m+2]}+c_{i, j}^{[10, m]}+ \\
& 2 c_{i, j}^{[12, m]}-2 c_{i, j}^{[11, m-1]}-2 c_{i, j}^{[11, m+1]}, \quad m=2,4,6,8,10 .
\end{aligned}
$$

Moreover, the $C^{2}$-smoothness across interior horizontal sides of the squares for $i=1, \ldots, n, j=1, \ldots, n-1$, reads as

$$
\begin{aligned}
c_{i, j}^{[m, 0]}= & c_{i, j+1}^{[m, 12]}, \quad m=0,2,4,6,8,10,12, \\
c_{i, j}^{[m, 1]}+c_{i, j+1}^{[m, 11]}= & c_{i, j}^{[m+1,0]}+c_{i, j}^{[m-1,0]}, \quad m=1,3,5,7,9,11, \\
c_{i, j+1}^{[m, 10]}= & c_{i, j}^{[m-2,0]}+c_{i, j}^{[m+2,0]}+c_{i, j}^{[m, 2]}+ \\
& 2 c_{i, j}^{[m, 0]}-2 c_{i, j}^{[m-1,1]}-2 c_{i, j}^{[m+1,1]}, \quad m=2,4,6,8,10 .
\end{aligned}
$$

Again, we only have to consider the substantial part of $\mathcal{D}$, i.e, $\eta \in \mathcal{D} \cap Q_{i, j}$, where $i, j \in\{2, \ldots, n-1\}$, since the proof for the remaining domain points is analogous with an appropriate use of the points in $\mathcal{M}^{*} \backslash\left(\widetilde{\mathcal{M}} \cup \mathcal{N}_{1} \cup \mathcal{N}_{2}\right)$.

We consider two cases.

Case 1: $i+j$ even. First, it is clear that $c_{\eta}=0$ for all $\eta \in \mathcal{D} \cap T_{i, j}^{[1]}$. We proceed by considering $T_{i, j}^{[2]}$. By (3.3), we have

$$
\begin{aligned}
c_{i, j}^{[m, m-2]} & =2 c_{i, j}^{[m-1, m-1]}-c_{i, j}^{[m-2, m]}=0, \quad m=2, \ldots, 7, \\
c_{i, j}^{[m+2, m-2]} & =4 c_{i, j}^{[m, m]}-4 c_{i, j}^{[m-1, m+1]}+c_{i, j}^{[m-2, m+2]}=0, \quad m=2, \ldots, 6,
\end{aligned}
$$

Using (3.4), we get successively

$$
\begin{aligned}
c_{i, j+1}^{[11,9]} & =c_{i, j+1}^{[12,10]}+c_{i, j+1}^{[12,8]}-c_{i+1, j+1}^{[1,9]}=0, \\
c_{i, j+1}^{[11,11]} & =c_{i, j+1}^{[12,12]}+c_{i, j+1}^{[12,10]}-c_{i+1, j+1}^{[1,11]}=0, \\
c_{i, j+1}^{[10,10]} & =c_{i+1, j+1}^{[2,10]}-c_{i, j+1}^{[12,8]}-c_{i, j+1}^{[12,12]}-2 c_{i, j+1}^{[12,10]}+2 c_{i, j+1}^{[11,9]}+2 c_{i, j+1}^{[11,11]}=0 .
\end{aligned}
$$

Condition (3.3) now implies $c_{i, j+1}^{[8,12]}=c_{i, j+1}^{[9,11]}=c_{i, j+1}^{[10,12]}=0$, and therefore by (3.4), we obtain successively

$$
\begin{aligned}
c_{i, j}^{[9,1]} & =c_{i, j}^{[8,0]}+c_{i, j}^{[10,0]}-c_{i, j+1}^{[9,11]}=0, \\
c_{i, j}^{[11,1]} & =c_{i, j}^{[10,0]}+c_{i, j}^{[12,0]}-c_{i, j+1}^{[11,11]}=0, \\
c_{i, j}^{[10,2]} & =c_{i, j+1}^{[10,10]}-c_{i, j}^{[8,0]}-c_{i, j}^{[12,0]}-2 c_{i, j}^{[10,0]}+2 c_{i, j}^{[9,1]}+2 c_{i, j}^{[11,1]}=0 .
\end{aligned}
$$




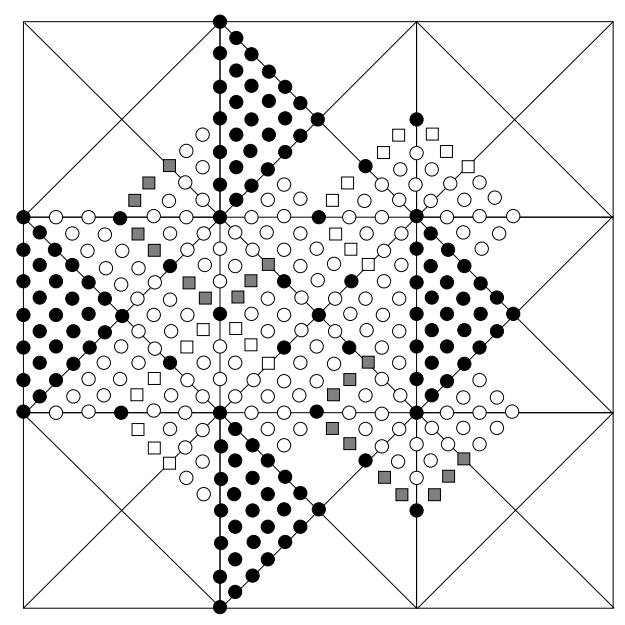

Fig. 7. BB-coefficients associated with points marked by boxes stand in relation.

Similar arguments give $c_{\eta}=0$ for all

$$
\eta \in\left\{\eta_{i, j}^{[11,3]}, \eta_{i, j}^{[12,2]}, \eta_{i, j}^{[12,4]}, \eta_{i+1, j}^{[1,1]}, \eta_{i+1, j}^{[1,3]}, \eta_{i+1, j}^{[2,0]}, \eta_{i+1, j}^{[2,2]}, \eta_{i+1, j}^{[3,1]}, \eta_{i+1, j+1}^{[3,11]}, \eta_{i+1, j}^{[4,0]}\right\} .
$$

By definition, we have

$$
\eta_{i, j}^{[6,0]}, \eta_{i, j}^{[9,3]}, \eta_{i, j}^{[12,6]} \in \mathcal{M}^{*} .
$$

It follows from this and the smoothness conditions (3.3), (3.4), and (3.5) that the coefficients $c_{\eta}$ with

$$
\eta \in\left\{\eta_{i, j}^{[7,1]}, \eta_{i, j}^{[8,2]}, \eta_{i, j}^{[10,4]}, \eta_{i, j}^{[11,5]}, \eta_{i+1, j}^{[1,5]}, \eta_{i+1, j}^{[2,4]}, \eta_{i+1, j}^{[3,3]}, \eta_{i, j+1}^{[7,11]}, \eta_{i, j+1}^{[8,10]}, \eta_{i, j+1}^{[9,9]}\right\}
$$

stand in linear relations. More precisely, we have $A \mathbf{c}=0$, where

$$
A=\left[\begin{array}{cccccccccc}
2 & -1 & 0 & 0 & 0 & 0 & 0 & 0 & 0 & 0 \\
4 & -4 & 1 & 0 & 0 & 0 & 0 & 0 & 0 & 0 \\
0 & 0 & 1 & 1 & 0 & 0 & 0 & 0 & 0 & 0 \\
0 & -1 & 2 & 0 & 1 & 0 & 0 & 0 & 0 & 0 \\
0 & 0 & 0 & 0 & 1 & 1 & 0 & 0 & 0 & 0 \\
0 & 0 & 0 & -1 & 4 & 0 & 1 & 0 & 0 & 0 \\
0 & 0 & 0 & 0 & 0 & 0 & 1 & 1 & 0 & 0 \\
0 & 0 & 0 & 0 & 0 & -1 & 2 & 0 & 1 & 0 \\
0 & 0 & 0 & 0 & 0 & 0 & 0 & 0 & -1 & 2 \\
0 & 0 & 0 & 0 & 0 & 0 & 0 & 1 & -4 & 4
\end{array}\right],
$$

and

$$
\mathbf{c}=\left(c_{i, j+1}^{[9,9]}, c_{i, j+1}^{[8,10]}, c_{i, j+1}^{[7,11]}, c_{i, j}^{[7,1]}, c_{i, j}^{[8,2]}, c_{i, j}^{[10,4]}, c_{i, j}^{[11,5]}, c_{i+1, j}^{[1,5]}, c_{i+1, j}^{[2,4]}, c_{i+1, j}^{[3,3]}\right)^{t}
$$

(see Fig. 7). 
Since $A$ is nonsingular, it follows that $\mathbf{c}=0$, and hence $c_{\eta}=0$ for all $\eta \in$ $\mathcal{D} \cap T_{i, j}^{[2]}$. Similarly, $c_{\eta}=0$ for all $\eta \in \mathcal{D} \cap T_{i, j}^{[4]}$. It remains to show that $c_{\eta}=0$ for all

$$
\eta \in\left\{\eta_{i, j}^{[8,6]}, \eta_{i, j}^{[9,5]}, \eta_{i, j}^{[9,7]}, \eta_{i, j}^{[10,6]}\right\}
$$

But, this follows from (3.3). Hence, $c_{\eta}=0$ for all $\eta \in \mathcal{D} \cap Q_{i, j}, i+j$ even.

Case 2: $i+j$ odd. Case 1 implies that that $c_{\eta}=0$ for all

$$
\eta \in \mathcal{D} \cap\left(T_{i+1, j}^{[1]} \cup T_{i, j-1}^{[2]} \cup T_{i-1, j}^{[3]} \cup T_{i, j+1}^{[4]}\right)
$$

Moreover, from the arguments given in Case 1, we obtain that $c_{\eta}=0$ for all $\eta \in \Gamma_{i, j}$, where

$$
\begin{aligned}
\Gamma_{i, j}= & \left\{\eta_{i, j}^{[m, \ell]}: m+\ell \leq 6\right\} \cup\left\{\eta_{i, j}^{[m, \ell]}: m+\ell \geq 18\right\} \\
& \cup\left\{\eta_{i, j}^{[m+\ell, \ell]}: \ell=0, \ldots, 12-m, m \in\{6, \ldots 10\}\right\} \\
& \cup\left\{\eta_{i, j}^{[\ell, m+\ell]}: \ell=0, \ldots, 12-m, m \in\{6, \ldots 10\}\right\} .
\end{aligned}
$$

It follows from (3.4) and (3.5) (for $m=6$ ) that $c_{\eta}=0$ for all

$$
\eta \in\left\{\eta_{i, j}^{[2,6]}, \eta_{i, j}^{[6,2]}, \eta_{i, j}^{[6,10]}, \eta_{i, j}^{[10,6]}\right\}
$$

We have $\mathcal{N}_{2} \subset \mathcal{M}^{*}$, and therefore the smoothness condition (3.3) (for $m=4,6,8$ ) successively imply that $c_{\eta}=0$ for all

$$
\eta \in\left\{\eta_{i, j}^{[3,5]}, \eta_{i, j}^{[3,7]}, \eta_{i, j}^{[5,5]}, \eta_{i, j}^{[5,7]}, \eta_{i, j}^{[7,3]}, \eta_{i, j}^{[7,9]}\right\}
$$

and $c_{\eta}=0$ for all

$$
\eta \in\left\{\eta_{i, j}^{[5,3]}, \eta_{i, j}^{[5,9]}, \eta_{i, j}^{[7,5]}, \eta_{i, j}^{[7,7]}, \eta_{i, j}^{[9,5]}, \eta_{i, j}^{[9,7]}\right\}
$$

The same argument (applying (3.3) for $m=5,7$ ) implies that $c_{\eta}=0$ for the remaining $\eta \in \mathcal{D} \cap Q_{i, j}$.

Proof of Theorem 2.4: In addition to the smoothness conditions (3.3)-(3.5), the splines in $\mathcal{S S}_{6}^{2}(\triangle)$ or $\mathcal{R S}_{6}^{2}(\triangle)$ satisfy the conditions (2.9) and (2.10) or (2.10) and (2.11), respectively. In both cases the number of these additional conditions is equal to $5 n^{2}$ which coincides with $\#\left(\mathcal{N}_{1} \cup \mathcal{N}_{2}\right)$. Since the dimension of $\mathcal{S}_{6}^{2}(\triangle)$ is equal to $19 n^{2}+24 n+6$, it follows that the dimensions of $\mathcal{S S}_{6}^{2}(\triangle)$ and $\mathcal{R S}_{6}^{2}(\triangle)$ are both at least $14 n^{2}+24 n+6$. Since

$$
\# \mathcal{M}=14 n^{2}+24 n+6
$$

it is again sufficient to check that $\mathcal{M}$ is a determining set. 
We consider first the space $\mathcal{S S}_{6}^{2}(\triangle)$. Let $s \in \mathcal{S S}_{6}^{2}(\triangle)$ be such that $c_{\eta}:=$ $c_{\eta}(s)=0$ for all $\eta \in \mathcal{M}$. Since $c_{\eta}=0$ for all $\eta \in \mathcal{D} \cap T_{i, j}^{[1]}, i+j$ even , it follows by the $C^{3}$ super-smoothness conditions (2.9) that $c_{\eta}=0$ for all

$$
\eta \in\left\{\eta_{i, j}^{[6,0]}, \eta_{i, j}^{[6,12]}, \eta_{i, j}^{[9,3]}, \eta_{i, j}^{[9,9]}, \eta_{i, j}^{[12,6]}\right\}, \quad i+j \text { even }
$$

Therefore, following the lines of the proof of Theorem 2.3, it can be seen that $c_{\eta}=0$ for all

$$
\eta \in \mathcal{D} \cap Q_{i, j}, \quad i+j \text { even, }
$$

and

$$
\eta \in \Gamma_{i, j} \cup\left\{\eta_{i, j}^{[2,6]}, \eta_{i, j}^{[6,2]}, \eta_{i, j}^{[6,10]}, \eta_{i, j}^{[10,6]}\right\}, \quad i+j \text { odd. }
$$

Now, the conditions (2.10) and (3.3) lead to five (independent) $3 \times 3$ linear systems of the form $B \mathbf{c}=0$, where $B$ is the non-singular matrix

$$
B=\left[\begin{array}{ccc}
1 & 2 & -1 \\
0 & 4 & -4 \\
0 & 8 & -12
\end{array}\right]
$$

and $\mathbf{c}$ is one of the 5 vectors

$$
\begin{array}{lll}
\left(c_{i, j}^{[5,9]}, c_{i, j}^{[4,8]}, c_{i, j}^{[3,7]}\right)^{t}, & \left(c_{i, j}^{[5,3]}, c_{i, j}^{[4,4]}, c_{i, j}^{[3,5]}\right)^{t}, & \left(c_{i, j}^{[9,5]}, c_{i, j}^{[8,4]}, c_{i, j}^{[7,3]}\right)^{t}, \\
\left(c_{i, j}^{[9,7]}, c_{i, j}^{[8,8]}, c_{i, j}^{[7,9]}\right)^{t}, & \left(c_{i, j}^{[7,7]}, c_{i, j}^{[6,6]}, c_{i, j}^{[5,5]}\right)^{t}, & i+j \text { odd } .
\end{array}
$$

It follows by arguments similar to those at the end of the proof of Theorem 2.3, that $c_{\eta}=0, \eta \in \mathcal{D}$.

Turning to the space $\mathcal{R S}_{6}^{2}(\triangle)$, we see that the same argument applies, the only difference being that (3.7) now follows from (2.11).

\section{$\S 4$. Locality and Stability of the Basis}

An important observation is that the basis splines for $\mathcal{S}_{3}^{1}(\triangle), \mathcal{S}_{6}^{2}(\triangle), \mathcal{S S}_{6}^{2}(\triangle)$ and $\mathcal{R S}_{6}^{2}(\triangle)$ corresponding to the MDS described in Section 2 have local support and are uniformly bounded. These properties will be used in Section 5 to obtain estimates for the error of our approximation operators.

\subsection{The Basis for $\mathcal{S}_{3}^{1}(\triangle)$}

As in Section 2.4, we denote by $B_{\eta}, \eta \in \mathcal{M}$, the basis splines for $\mathcal{S}_{3}^{1}(\triangle)$ associated with the MDS $\mathcal{M}$ defined in Section 2.2. 


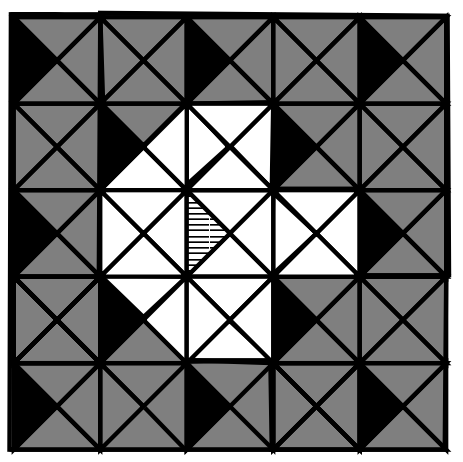

Fig. 8. Supports of $C^{1}$ basis splines associated with points in $\widetilde{\mathcal{M}}$.

Lemma 4.1. The basis splines $B_{\eta}, \eta \in \mathcal{M}$, for $\mathcal{S}_{3}^{1}(\triangle)$ are 3-locally supported.

Proof: For simplicity we only consider the case when $\eta \in \widetilde{\mathcal{M}}$ and $\eta$ lies sufficiently far from the boundary of $\Omega$, i.e. we assume that $\eta \in \mathcal{D} \cap T_{i, j}^{[1]}$ for some $3 \leq i, j \leq n-2$, with $i+j$ even. If $\eta$ is located near the boundary our arguments can be appropriately modified, and supports do not become larger.

First, it is obvious that $c_{\xi}\left(B_{\eta}\right)=0$ for all

$$
\xi \in \mathcal{D} \cap T_{i^{\prime}, j^{\prime}}^{[1]}, \quad\left(i^{\prime}, j^{\prime}\right) \neq(i, j), \quad i^{\prime}+j^{\prime} \text { even }
$$

Therefore, it follows from the proof of Theorem 2.2 that $c_{\xi}\left(B_{\eta}\right)=0$ for all

$$
\xi \in \mathcal{D} \cap \bigcup_{\substack{i^{\prime} \neq i-1, i, i+1 \\ j^{\prime} \neq j-1, j, j+1}} Q_{i^{\prime}, j^{\prime}}
$$

Moreover, $c_{\xi}\left(B_{\eta}\right)=0$ for all

$$
\xi \in \mathcal{D} \cap\left(Q_{i+1, j-1} \cup Q_{i+1, j+1} \cup T_{i-1, j-1}^{[1]} \cup T_{i-1, j-1}^{[2]} \cup T_{i-1, j+1}^{[1]} \cup T_{i-1, j+1}^{[4]}\right) .
$$

Therefore, it follows that

$$
\begin{aligned}
\operatorname{supp} B_{\eta} \subseteq & Q_{i, j} \cup Q_{i-1, j} \cup Q_{i+1, j} \cup Q_{i, j-1} \cup Q_{i, j+1} \\
& \cup T_{i-1, j-1}^{[2]} \cup T_{i-1, j-1}^{[3]} \cup T_{i-1, j+1}^{[3]} \cup T_{i-1, j+1}^{[4]},
\end{aligned}
$$

which implies that $\operatorname{supp} B_{\eta} \subseteq \operatorname{star}^{3}(v)$, where $v$ is the central vertex of $Q_{i, j}$.

Fig. 8 illustrates the supports of the basis splines associated with the domain points from $\widetilde{\mathcal{M}}$, i.e. the points lying in the crosshatched triangle. Outside of the white area these 10 basis splines vanish. 

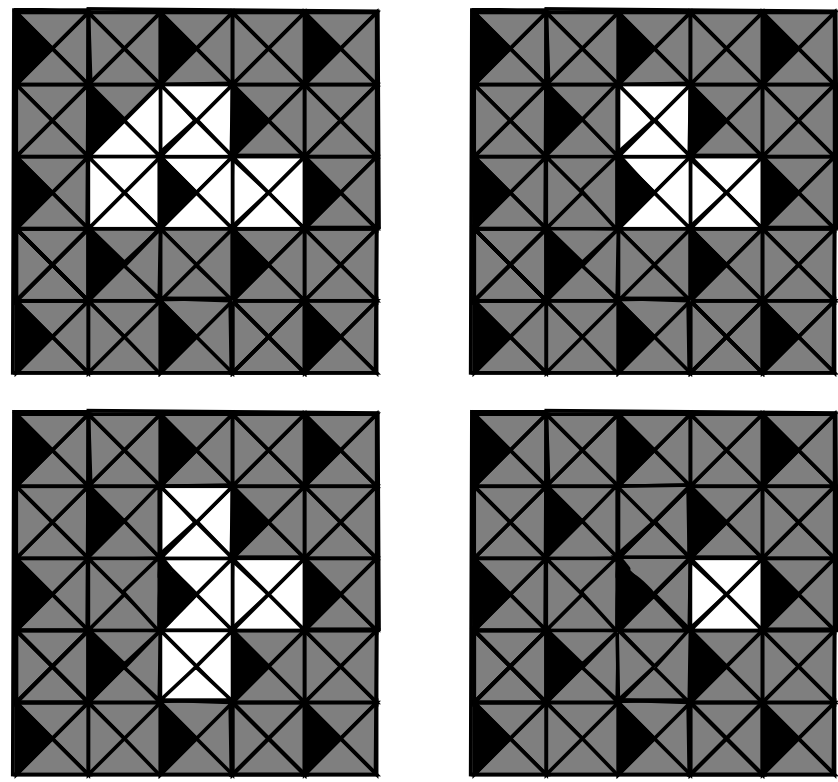

Fig. 9. Supports of $C^{2}$ basis splines associated with points in $\mathcal{N}_{1} \cup \mathcal{N}_{2}$.

Lemma 4.2. The basis splines $B_{\eta}, \eta \in \mathcal{M}$, for $\mathcal{S}_{3}^{1}(\triangle)$ are uniformly bounded, i.e., there exists an absolute constant $K$ such that

$$
\left\|B_{\eta}\right\|_{\infty} \leq K, \quad \text { all } \eta \in \mathcal{M}
$$

Proof: Obviously, the BB-coefficients of $B_{\eta}$ can be computed using the smoothness conditions (3.1) and (3.2) and following the lines of the proof of Theorem 2.2. It is easy to see that every BB-coefficient $c_{\xi}, \xi \in \mathcal{D} \backslash \mathcal{M}$, of $B_{\eta}$ is computed by using at most 7 smoothness conditions (3.1), (3.2). Since each application of a condition of this type may increase the magnitude of $c_{\xi}$ at most by the factor 3 , we get the rough estimate

$$
\left|c_{\xi}\right| \leq 21, \quad \text { all } \xi \in \mathcal{D} .
$$

(A more careful examination shows that $\left|c_{\xi}\right| \leq 4$.) The desired estimation (4.1) now follows since in view of the well-known property of the Bernstein-Bézier representation, $\left\|B_{\eta}\right\|_{\infty} \leq \max _{\xi \in \mathcal{D}}\left|c_{\xi}\right|$.

\subsection{The Basis for $\mathcal{S}_{6}^{2}(\triangle)$}

Let us denote by $B_{\eta}^{*}, \eta \in \mathcal{M}^{*}$, the basis splines for $\mathcal{S}_{6}^{2}(\triangle)$ associated with the MDS $\mathcal{M}$ defined in Section 2.3.

Lemma 4.3. The basis splines $B_{\eta}^{*}, \eta \in \mathcal{M}^{*}$, for $\mathcal{S}_{6}^{2}(\triangle)$ are 3-locally supported.

Proof: Again, for simplicity we only consider the case when $\eta \in \widetilde{\mathcal{M}} \cup \mathcal{N}_{1} \cup \mathcal{N}_{2}$ and $\eta$ lies sufficiently far from the boundary of $\Omega$, since for $\eta$ located near the boundary, the arguments given below can be appropriately modified and supports do not become larger. 
We consider two cases.

Case 1: Let $\eta \in \mathcal{D} \cap T_{i, j}^{[1]}$ or $\eta \in\left\{\eta_{i, j}^{[6,0]}, \eta_{i, j}^{[6,12]}, \eta_{i, j}^{[9,3]}, \eta_{i, j}^{[9,9]}, \eta_{i, j}^{[12,6]}\right\}$ for some $3 \leq$ $i, j \leq n-2$, with $i+j$ even, i.e. $\eta \in \widetilde{\mathcal{M}} \cup \mathcal{N}_{1}$. This case is analogous to the proof of Lemma 4.1. Using the arguments from the proof of Theorem 2.3, it can be seen that

$$
\begin{aligned}
\operatorname{supp} B_{\eta}^{*} \subseteq & Q_{i, j} \cup Q_{i-1, j} \cup Q_{i+1, j} \cup Q_{i, j-1} \cup Q_{i, j+1} \\
& \cup T_{i-1, j-1}^{[2]} \cup T_{i-1, j-1}^{[3]} \cup T_{i-1, j+1}^{[3]} \cup T_{i-1, j+1}^{[4]},
\end{aligned}
$$

which implies that $\operatorname{supp} B_{\eta}^{*} \subseteq \operatorname{star}^{3}(v)$, where $v$ is the central vertex of $Q_{i, j}$.

Case 2: Let $\eta \in\left\{\eta_{i, j}^{[4,4]}, \eta_{i, j}^{[4,8]}, \eta_{i, j}^{[6,6]}, \eta_{i, j}^{[8,4]}, \eta_{i, j}^{[8,8]}\right\}$ for some $2 \leq i, j \leq n-1$, with $i+j$ odd, i.e. $\eta \in \mathcal{N}_{2}$. First, it is obvious that $c_{\xi}\left(B_{\eta}^{*}\right)=0$ for all

$$
\xi \in \mathcal{D} \cap T_{i^{\prime}, j^{\prime}}^{[1]}, \quad\left(i^{\prime}, j^{\prime}\right) \neq(i, j), \quad i^{\prime}+j^{\prime} \text { even. }
$$

Moreover, $c_{\xi}\left(B_{\eta}^{*}\right)=0$ for all $\xi \in \mathcal{N}_{1}$. Therefore, it follows from the proof of Theorem 2.3 that $c_{\xi}\left(B_{\eta}^{*}\right)=0$ for all

$$
\xi \in \mathcal{D} \cap \bigcup_{\substack{i^{\prime} \neq i \\ j^{\prime} \neq j}} Q_{i^{\prime}, j^{\prime}}
$$

Hence,

$$
\operatorname{supp} B_{\eta}^{*} \subseteq Q_{i, j}
$$

which implies that $\operatorname{supp} B_{\eta}^{*} \subseteq \operatorname{star}(v)$, where $v$ is the central vertex of $Q_{i, j}$.

Fig. 8 illustrates the support of the basis splines of $\mathcal{S}_{6}^{2}(\triangle)$ associated with the domain points from $\widetilde{\mathcal{M}}$, i.e. the points inside the crosshatched triangle. Outside of the white area these 28 basis splines vanish. Moreover, Fig. 9 shows supports (white areas) of the basis splines of $\mathcal{S}_{6}^{2}(\triangle)$ associated with the domain points from $\mathcal{N}_{1}$ and $\mathcal{N}_{2}$. Here, the lower right figure illustrates the support of the basis splines associated with points from $\mathcal{N}_{2}$, while the remaining figures show some typical supports of the basis splines associated with points from $\mathcal{N}_{1}$ (again, outside the white area the corresponding basis splines vanish).

Lemma 4.4. The basis splines $B_{\eta}^{*}, \eta \in \mathcal{M}^{*}$ for $\mathcal{S}_{6}^{2}(\triangle)$ are uniformly bounded, i.e., there exists an absolute constant $K^{*}$ such that

$$
\left\|B_{\eta}^{*}\right\|_{\infty} \leq K^{*}, \quad \text { all } \eta \in \mathcal{M}^{*}
$$

Proof: The BB-coefficients $c_{\xi}$ of $B_{\eta}^{*}, \eta \in \mathcal{M}^{*}$ can be computed using the smoothness conditions (3.3), (3.4), (3.5) and following the lines of the proof of Theorem 2.3 with the difference that $c_{\eta}=1$, while $c_{\xi}=0$ for all $\xi \in \mathcal{M}^{*} \backslash\{\eta\}$. Again, it suffices to consider the case where $\eta \in \widetilde{\mathcal{M}} \cup \mathcal{N}_{1} \cup \mathcal{N}_{2}$ lies sufficiently far from the boundary. 
Let $\eta \in \mathcal{D} \cap T_{i, j}^{[1]}$, where $i+j$ even. The smoothness conditions (3.3), (3.4), (3.5) across the edges of $T_{i, j}^{[1]}$ increase the magnitude of the involved coefficients $c_{\xi}$ at most by the factor 9 . We proceed by considering the coefficients $c_{\xi}$ which are determined by a system of the form $A \mathbf{c}=b$, where $A$ is the matrix from the proof of Theorem 2.3. Solving this system further increases the magnitude of the coefficients by at most the factor

$$
\left\|A^{-1}\right\|_{\infty}=21 / 4
$$

The proof of Theorem 2.3 shows that the smoothness conditions (3.3), (3.4), (3.5) have to be applied three more times to determine the remaining possibly non-zero coefficients $c_{\xi}$ of $B_{\eta}^{*}$. Hence, we get the rough estimate

$$
\left|c_{\xi}\right| \leq 21 \cdot 9^{4} / 4, \quad \text { all } \xi \in \mathcal{D}
$$

in this case. The remaining cases $\eta \in \mathcal{N}_{1} \cup \mathcal{N}_{2}$ are simpler, and we have the rough estimates

$$
\left|c_{\xi}\right| \leq 21 \cdot 9^{3} / 4, \quad \text { all } \xi \in \mathcal{D},
$$

for $\eta \in \mathcal{N}_{1}$, and

$$
\left|c_{\xi}\right| \leq 81, \quad \text { all } \xi \in \mathcal{D}
$$

for $\eta \in \mathcal{N}_{2}$, respectively.

The desired estimation (4.2) now follows since in view of the well-known property of the Bernstein-Bézier representation, $\left\|B_{\eta}^{*}\right\|_{\infty} \leq \max _{\xi \in \mathcal{D}}\left|c_{\xi}\right|$.

\subsection{The Basis for $\mathcal{S S}_{6}^{2}(\triangle)$}

We now examine the support of the basis splines $B_{\eta}, \eta \in \mathcal{M}$, for $\mathcal{S S}_{6}^{2}(\triangle)$, associated with the MDS $\mathcal{M}$ of Section 2.3. Clearly, every $B_{\eta}$, as an element of $\mathcal{S}_{6}^{2}(\triangle)$, can be written as

$$
B_{\eta}=\sum_{\xi \in \mathcal{M}^{*}} \alpha_{\xi} B_{\xi}^{*}
$$

where $\alpha_{\xi}, \xi \in \mathcal{M}^{*}$, are the corresponding BB-coefficients of $B_{\eta}$. Therefore,

$$
B_{\eta}=B_{\eta}^{*}+\sum_{\xi \in \mathcal{N}_{1} \cup \mathcal{N}_{2}} \alpha_{\xi} B_{\xi}^{*}
$$

and hence the support of $B_{\eta}$ is contained in the union of $\operatorname{supp} B_{\eta}^{*}$ and the supports of the basis splines $B_{\xi}^{*}, \xi \in \mathcal{N}_{1} \cup \mathcal{N}_{2}$, corresponding to nonzero $\alpha_{\xi}$ in (4.3).

Thus, to show that $B_{\eta}$ has a local support, we have to analyse how the coefficients $\alpha_{\xi}, \xi \in \mathcal{N}_{1} \cup \mathcal{N}_{2}$, are computed by (2.9) and (2.10) (see the proof of Theorem 2.4 in Section 3), and check that $\alpha_{\xi}=0$ as soon as $\xi$ lies at a substantial distance from $\eta$. We will see that although in general the support of $B_{\eta}$ is slightly larger than the support of $B_{\eta}^{*}$, it remains contained in $\operatorname{star}^{3}(v)$ for an appropriate vertex $v$ of $\triangle$. 
Lemma 4.5. The basis splines $B_{\eta}, \eta \in \mathcal{M}$, for $\mathcal{S S}_{6}^{2}(\triangle)$ are 3-locally supported.

Proof: For simplicity we only consider the case when $\eta \in \widetilde{\mathcal{M}}$ lies sufficiently far from the boundary of $\Omega$, i.e. $\eta \in \mathcal{D} \cap Q_{i, j}$ with $3 \leq i, j \leq n-2$. For $\eta$ located closer to the boundary our arguments have to be appropriately modified, where it can be seen that the supports are not larger than in the cases below.

First, we recall that

$$
\operatorname{supp} B_{\eta}^{*}=Q_{i, j}, \quad \xi \in \mathcal{N}_{2} \cap Q_{i, j}, \quad i+j \text { odd }
$$

see Case 2 of the proof of Lemma 4.3. Moreover, by (2.10) and the proofs of Theorems 2.3 and 2.4, it is easy to see that the computation of $\alpha_{\xi}, \xi \in \mathcal{N}_{2} \cap Q_{i, j}$, for some $i, j$, with $i+j$ odd, only involves the BB-coefficients of $B_{\eta}$ corresponding to domain points in $\mathcal{D} \cap Q_{i, j}$. Therefore, the terms in (4.3) corresponding to $\xi \in \mathcal{N}_{2}$ do not increase the support of $B_{\eta}$, and we concentrate in what follows on $\xi \in \mathcal{N}_{1}$.

We consider six cases.

Case 1: Let

$$
\begin{gathered}
\eta \in\left\{\eta_{i, j}^{[0,0]}, \eta_{i, j}^{[0,2]}, \eta_{i, j}^{[0,4]}, \eta_{i, j}^{[1,1]}, \eta_{i, j}^{[1,3]}, \eta_{i, j}^{[1,9]}, \eta_{i, j}^{[1,11]}, \eta_{i, j}^{[2,2]}, \eta_{i, j}^{[2,6]}\right. \\
\left.\eta_{i, j}^{[2,10]}, \eta_{i, j}^{[3,5]}, \eta_{i, j}^{[3,7]}, \eta_{i, j}^{[4,6]}\right\}, \quad i+j \text { even. }
\end{gathered}
$$

It follows from the smoothness conditions (3.3), (3.4), (3.5), and (2.9) that $\alpha_{\xi}=0$, $\xi \in \mathcal{N}_{1}$. The same arguments as in the proof of Lemma 4.3 (in Case 1) show that in these cases we have

$$
\begin{aligned}
\operatorname{supp} B_{\eta} \subseteq & Q_{i, j} \cup Q_{i-1, j} \cup Q_{i+1, j} \cup Q_{i, j-1} \cup Q_{i, j+1} \\
& \cup T_{i-1, j-1}^{[2]} \cup T_{i-1, j-1}^{[3]} \cup T_{i-1, j+1}^{[3]} \cup T_{i-1, j+1}^{[4]},
\end{aligned}
$$

which implies that $B_{\eta} \subseteq \operatorname{star}^{3}(v)$, where $v$ is the central vertex of $Q_{i, j}$.

Case 2: Let

$$
\eta \in\left\{\eta_{i, j}^{[0,8]}, \eta_{i, j}^{[0,10]}, \eta_{i, j}^{[0,12]}\right\}, \quad i+j \text { even. }
$$

It follows from the smoothness condition (3.3), (3.4), (3.5), and (2.9) that in the representation (4.3) of $B_{\eta}$ we have $\alpha_{\xi_{0}} \neq 0$, where $\xi_{0}=\xi_{i, j-1}^{[0,6]}=\xi_{i-1, j-1}^{[12,6]}$. Moreover, $\alpha_{\xi}=0, \xi \in \mathcal{N}_{1} \backslash\left\{\xi_{0}\right\}$. A close inspection and the arguments given in the proof of Lemma 4.3 (in Case 1) show that in these cases we have

$$
\operatorname{supp} B_{\eta} \subseteq Q_{i, j} \cup Q_{i, j-1} \cup Q_{i-1, j} \cup Q_{i-1, j-1} \cup Q_{i-1, j-2}
$$

which implies that $B_{\eta} \subseteq \operatorname{star}^{3}(v)$, where $v$ is the upper left vertex of $Q_{i, j}$.

Case 3: Let

$$
\eta \in\left\{\eta_{i, j}^{[1,5]}, \eta_{i, j}^{[1,7]}, \eta_{i, j}^{[2,4]}, \eta_{i, j}^{[2,8]}, \eta_{i, j}^{[4,4]}, \eta_{i, j}^{[4,8]}, \eta_{i, j}^{[5,5]}, \eta_{i, j}^{[5,7]}\right\}, \quad i+j \text { even. }
$$



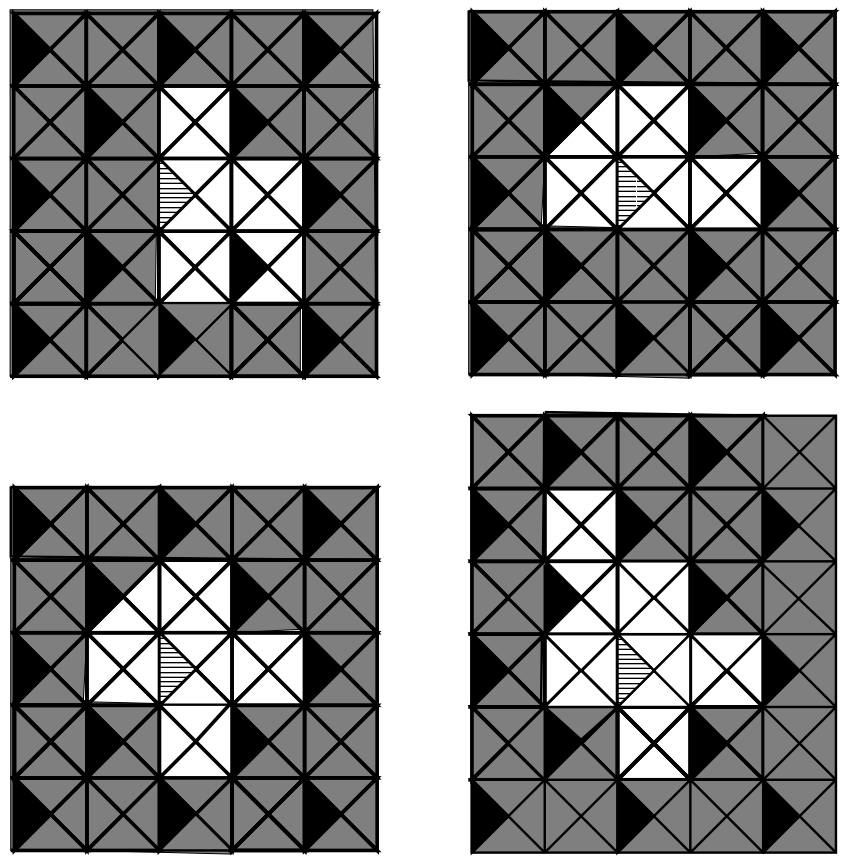

Fig. 10. Supports of some basis splines from $\mathcal{S S}_{6}^{2}(\triangle)$.

We consider only the cases $\eta \in\left\{\eta_{i, j}^{[1,5]}, \eta_{i, j}^{[2,4]}\right\}$ since the remaining cases are similar. In these cases, it follows from the smoothness condition (3.3), (3.4), (3.5), and (2.9) that in the representation (4.3) of $B_{\eta}$ we have $\alpha_{\xi_{0}} \neq 0$, where $\xi_{0}=\xi_{i, j}^{[6,0]}$. Moreover, $\alpha_{\xi}=0, \xi \in \mathcal{N}_{1} \backslash\left\{\xi_{0}\right\}$. A close inspection and the arguments given in the proof of Lemma 4.3 (in Case 1) show that in these cases we have

$$
\operatorname{supp} B_{\eta} \subseteq Q_{i, j} \cup Q_{i, j+1} \cup Q_{i-1, j} \cup Q_{i+1, j} \cup T_{i-1, j+1}^{[3]} \cup T_{i-1, j+1}^{[4]},
$$

which implies that $B_{\eta} \subseteq \operatorname{star}^{3}(v)$, where $v$ is the central vertex of $Q_{i, j}$.

Case 4: Let

$$
\eta \in\left\{\eta_{i, j}^{[3,3]}, \eta_{i, j}^{[3,9]}\right\}, \quad i+j \text { even }
$$

We consider only the case $\eta=\eta_{i, j}^{[3,3]}$ since the case $\eta=\eta_{i, j}^{[3,9]}$ is completely symetric. In this case, it follows from the smoothness condition (3.3), (3.4), (3.5), and (2.9) that in the representation (4.3) of $B_{\eta}$ we have $\alpha_{\xi} \neq 0$, where

$$
\xi \in\left\{\xi_{i, j}^{[6,0]}, \xi_{i, j}^{[9,9]}\right\} .
$$

Moreover, $\alpha_{\xi}=0, \xi \in \mathcal{N}_{1} \backslash\left\{\xi_{i, j}^{[6,0]}, \xi_{i, j}^{[9,9]}\right\}$. A close inspection and the arguments given in the proof of Lemma 4.3 (Case 1 ) show that in this case we have

$$
\operatorname{supp} B_{\eta} \subseteq Q_{i, j} \cup Q_{i, j-1} \cup Q_{i, j+1} \cup Q_{i-1, j} \cup Q_{i+1, j} \cup T_{i-1, j+1}^{[3]} \cup T_{i-1, j+1}^{[4]},
$$

which implies that $B_{\eta} \subseteq \operatorname{star}^{3}(v)$, where $v$ is the central vertex of $Q_{i, j}$. 
Case 5: Let $\eta=\eta_{i, j}^{[6,6]}, i+j$ even. In this case, it follows from the smoothness condition (3.3), (3.4), (3.5), and (2.9) that in the representation (4.3) of $B_{\eta}$ we have $\alpha_{\xi} \neq 0$, where

$$
\xi \in\left\{\xi_{i, j}^{[9,3]}, \xi_{i, j}^{[9,9]}\right\} .
$$

Moreover, $\alpha_{\xi}=0, \xi \in \mathcal{N}_{1} \backslash\left\{\xi_{i, j}^{[9,3]}, \xi_{i, j}^{[9,9]}\right\}$. A close inspection and the arguments given in the proof of Lemma 4.3 (Case 1 ) show that in this case we have

$$
\operatorname{supp} B_{\eta} \subseteq Q_{i, j} \cup Q_{i, j-1} \cup Q_{i, j+1} \cup Q_{i+1, j}
$$

which implies that $B_{\eta} \subseteq \operatorname{star}^{3}(v)$, where $v$ is the central vertex of $Q_{i, j}$.

Case 6: Let $\eta=\eta_{i, j}^{[0,6]}, i+j$ even. In this case, it follows from the smoothness condition (3.3), (3.4), (3.5), and (2.9) that in the representation (4.3) of $B_{\eta}$ we have $\alpha_{\xi} \neq 0$, where

$$
\xi \in\left\{\xi_{i, j-1}^{[0,6]}, \xi_{i, j}^{[6,0]}, \xi_{i, j}^{[6,12]},\right\} .
$$

Moreover, $\alpha_{\xi}=0, \xi \in \mathcal{N}_{1} \backslash\left\{\xi_{i, j-1}^{[0,6]}, \xi_{i, j}^{[6,0]}, \xi_{i, j}^{[6,12]}\right\}$. A close inspection and the arguments given in the proof of Lemma 4.3 (Case 1) show that in this case we have

$$
\operatorname{supp} B_{\eta} \subseteq Q_{i, j} \cup Q_{i, j-1} \cup Q_{i, j+1} \cup Q_{i-1, j} \cup Q_{i-1, j-1} \cup Q_{i-1, j-2} \cup Q_{i+1, j}
$$

which implies that $B_{\eta} \subseteq \operatorname{star}^{3}(v)$, where $v$ is the upper left vertex of $Q_{i, j}$.

Fig. 10 shows areas (white and crosshatched triangles) which contain typical supports of basis splines of $\mathcal{S S}_{6}^{2}(\triangle)$ associated with the domain points from $\mathcal{M}$ (the Cases 2-4, and 6 from the proof of Theorem 4.5 are illustrated in Fig. 10).

Lemma 4.6. The basis splines $B_{\eta}, \eta \in \mathcal{M}$ of $\mathcal{S S}_{6}^{2}(\triangle)$ are uniformly bounded, i.e., there exists an absolute constant $K$ such that

$$
\left\|B_{\eta}\right\|_{\infty} \leq K, \quad \text { all } \eta \in \mathcal{M}
$$

Proof: The proof of Lemma 4.5 shows that

$$
B_{\eta}=B_{\eta}^{*}+\sum_{\xi \in N_{1}} \alpha_{\xi} B_{\xi}^{*}+\sum_{\xi \in N_{2}} \alpha_{\xi} B_{\xi}^{*}
$$

where $N_{1} \subseteq \mathcal{N}_{1}$ with $\# N_{1} \leq 3$, and $N_{2} \subseteq \mathcal{N}_{2}$ with $\# N_{2} \leq 25$. It follows from Lemma 4.4 that the basis splines $B_{\xi}^{*}, \xi \in\{\eta\} \cup N_{1} \cup N_{2}$ of $\mathcal{S}_{6}^{2}(\triangle)$ are uniformly bounded, and therefore it suffices to show that the coefficients $\alpha_{\xi}, \xi \in N_{1} \cup N_{2}$ are bounded by some fixed constant.

This can be seen easily for the coefficients $\alpha_{\xi}, \xi \in N_{1}$, since each application of (2.9) increases the magnitude of the corresponding BB-coefficient of a basis spline 
$B_{\eta}$ at most by the factor 27. Moreover, the splines $B_{\xi}^{*}$ with $\xi \in N_{2}$ only have influence on the BB-coefficients $c_{\xi^{\prime}}$ of $B_{\eta}$ with

$$
\xi^{\prime} \in\left(\left\{\eta_{i, j}^{[m, \ell]}, 3 \leq m, \ell \leq 9\right\} \backslash\left\{\eta_{i, j}^{[3,3]}, \eta_{i, j}^{[3,9]}, \eta_{i, j}^{[9,3]}, \eta_{i, j}^{[9,9]}\right\}\right)
$$

where $i+j$ odd, and $\xi \in \mathcal{D} \cap Q_{i, j}$. It follows from the proof of Theorem 2.4 that these $\mathrm{BB}$-coefficients of $B_{\eta}$ are determined as the solution of a linear system of the form $B \mathbf{c}=d$, where $B$ is the matrix in (3.8), or by applying the smoothness conditions (3.3). Arguing as in the proof of Lemma 4.4, we see that these BB-coefficients are uniformly bounded, and hence the coefficients $\alpha_{\xi}, \xi \in N_{2}$, in (4.5) are bounded by some fixed constant.

\subsection{The Basis for $\mathcal{R S}_{6}^{2}(\triangle)$}

The examination of the supports of the basis splines $B_{\eta}, \eta \in \mathcal{M}$, for $\mathcal{R S}_{6}^{2}(\triangle)$ is quite similar to $\mathcal{S S}_{6}^{2}(\triangle)$. In particular, we again have the representation (4.3), with some new coefficients $\alpha_{\xi}$.

Lemma 4.7. The basis splines $B_{\eta}, \eta \in \mathcal{M}$, for $\mathcal{R S}_{6}^{2}(\triangle)$ are 3-locally supported.

Proof: Again, we restrict ouselves to the case when $\eta \in \widetilde{\mathcal{M}}$ lies sufficiently far from the boundary of $\Omega$, i.e. $\eta \in \mathcal{D} \cap Q_{i, j}$ with $3 \leq i, j \leq n-2$. Since the terms in (4.3) corresponding to $\xi \in \mathcal{N}_{2}$ do not increase the support of $B_{\eta}$ (by the same argument as in the proof of Lemma 4.5), we only have to analyse the case $\xi \in \mathcal{N}_{1}$.

We consider four cases.

Case 1: Let

$$
\eta \in\left\{\eta_{i, j}^{[0,6]}, \eta_{i, j}^{[1,5]}, \eta_{i, j}^{[1,7]}, \eta_{i, j}^{[2,4]}, \eta_{i, j}^{[2,6]}, \eta_{i, j}^{[2,8]}, \eta_{i, j}^{[3,5]}, \eta_{i, j}^{[3,7]}, \eta_{i, j}^{[4,6]}\right\}, \quad i+j \text { even. }
$$

It follows from the smoothness conditions (3.3), (3.4), (3.5), and (2.11) that $\alpha_{\xi}=0$, $\xi \in \mathcal{N}_{1}$. The same arguments as in the proof of Lemma 4.3 (in Case 1) show that in these cases we have

$$
\begin{aligned}
\operatorname{supp} B_{\eta} \subseteq & Q_{i, j} \cup Q_{i-1, j} \cup Q_{i+1, j} \cup Q_{i, j-1} \cup Q_{i, j+1} \\
& \cup T_{i-1, j-1}^{[2]} \cup T_{i-1, j-1}^{[3]} \cup T_{i-1, j+1}^{[3]} \cup T_{i-1, j+1}^{[4]}
\end{aligned}
$$

which implies that $B_{\eta} \subseteq \operatorname{star}^{3}(v)$, where $v$ is the central vertex of $Q_{i, j}$.

Case 2: Let

$$
\begin{gathered}
\eta \in\left\{\eta_{i, j}^{[0,0]}, \eta_{i, j}^{[0,2]}, \eta_{i, j}^{[0,4]}, \eta_{i, j}^{[0,8]}, \eta_{i, j}^{[0,10]}, \eta_{i, j}^{[0,12]}, \eta_{i, j}^{[1,1]}, \eta_{i, j}^{[1,3]}\right. \\
\left.\eta_{i, j}^{[1,9]}, \eta_{i, j}^{[1,11]}, \eta_{i, j}^{[2,2]}, \eta_{i, j}^{[2,11]}\right\}, \quad i+j \text { even. }
\end{gathered}
$$




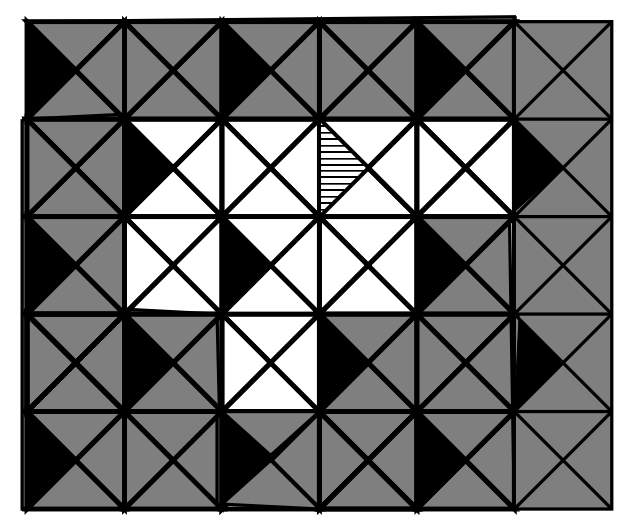

Fig. 11. Supports of some basis splines from $\mathcal{R S}_{6}^{2}(\triangle)$.

We consider the cases $\eta=\eta_{i, j}^{[m, \ell]}$ with $m+\ell \leq 4$, since the remaining cases are completely symetric. In this case, it follows from the smoothness condition (3.3), (3.4), (3.5), and (2.11) that in the representation (4.3) of $B_{\eta}$ we have $\alpha_{\xi} \neq 0$, where

$$
\xi \in \Lambda:=\left\{\eta_{i, j}^{[6,0]}, \eta_{i-1, j+1}^{[6,12]}, \eta_{i-1, j+1}^{[9,9]}, \eta_{i-1, j+1}^{[12,6]}\right\} .
$$

Moreover, $\alpha_{\xi}=0, \xi \in \mathcal{N}_{1} \backslash \Lambda$. A close inspection and the arguments given in the proof of Lemma 4.3 (in Case 1) show that in these cases we have

$$
\begin{aligned}
\operatorname{supp} B_{\eta} \subseteq Q_{i, j} \cup Q_{i, j+1} \cup Q_{i-2, j} \cup Q_{i-2, j+1} \cup \\
Q_{i-1, j} \cup Q_{i-1, j+1} \cup Q_{i-1, j+2} \cup Q_{i+1, j}
\end{aligned}
$$

which implies that $B_{\eta} \subseteq \operatorname{star}^{3}(v)$, where $v$ is the lower left vertex of $Q_{i, j}$.

Case 3: Let

$$
\eta \in\left\{\eta_{i, j}^{[3,3]}, \eta_{i, j}^{[3,9]}, \eta_{i, j}^{[4,4]}, \eta_{i, j}^{[4,8]}, \eta_{i, j}^{[5,5]}, \eta_{i, j}^{[5,7]}\right\}, \quad i+j \text { even }
$$

We consider the cases $\eta=\eta_{i, j}^{[m, m]}, m=3,4,5$, since the remaining cases are completely symmetric. In this case, it follows from the smoothness condition (3.3), (3.4), (3.5), and (2.11) that in the representation (4.3) of $B_{\eta}$ we have $\alpha_{\xi_{0}} \neq 0$, where $\xi_{0}=\eta_{i, j}^{[9,9]}$ and $\alpha_{\xi}=0, \xi \in \mathcal{N}_{1} \backslash\left\{\xi_{0}\right\}$. A close inspection and the arguments given in the proof of Lemma 4.3 (in Case 1) show that in these cases we have

$$
\operatorname{supp} B_{\eta} \subseteq Q_{i, j} \cup Q_{i-1, j} \cup Q_{i+1, j} \cup Q_{i, j-1} \cup Q_{i, j+1} \cup T_{i-1, j+1}^{[3]} \cup T_{i-1, j+1}^{[4]},
$$

which implies that $B_{\eta} \subseteq \operatorname{star}^{3}(v)$, where $v$ is the central vertex of $Q_{i, j}$.

Case 4: Let $\eta=\eta_{i, j}^{[6,6]}, i+j$ even. This case is analogous to Case 5 in the proof of Lemma 4.5.

The basis splines from $\mathcal{R S}_{6}^{2}(\triangle)$ treated in Case 2 of the proof of Lemma 4.7 are contained in the white area in Fig. 11 (together with the crosshatched triangle). 
Lemma 4.8. The basis splines $B_{\eta}, \eta \in \mathcal{M}$ of $\mathcal{R S}_{6}^{2}(\triangle)$ are uniformly bounded, i.e., there exists an absolute constant $K$ such that

$$
\left\|B_{\eta}\right\|_{\infty} \leq K, \quad \text { all } \eta \in \mathcal{M}
$$

Proof: As in the proof of Lemma 4.6, it suffices to show that the coefficients $\alpha_{\xi}$, $\xi \in N_{1} \cup N_{2}$ are bounded by some fixed constant, where $N_{1}$ and $N_{2}$ are appropriate subsets of $\mathcal{N}_{1}$ and $\mathcal{N}_{2}$, respectively. This follows for $\xi \in N_{1}$ since each application of (2.11) increases the magnitude of the corresponding BB-coefficient of a basis spline $B_{\eta}$ at most by the factor $11 / 5$. We note that we now have $\# N_{1} \leq 4$. The case of $\xi \in N_{2}$ can be treated exactly as in the proof of Lemma 4.6.

\section{$\S 5$. Error Bounds}

The purpose of this section is to give error bounds for our approximation operators defined in Section 2.4 assuming that the scattered data come from a sufficiently smooth function and appropriate local approximations are used.

Let $\mathcal{S}$ be one of the spaces $\mathcal{S}_{3}^{1}(\triangle), \mathcal{S S}_{6}^{2}(\triangle)$ or $\mathcal{R S}_{6}^{2}(\triangle)$, and let

$$
\left\{B_{\eta}: \eta \in \mathcal{M}_{\mathcal{S}}\right\}
$$

be the basis for $\mathcal{S}$ defined in Section 2.4 using the corresponding MDS of Sections 2.2 and 2.3. The results of Section 4 imply that (5.1) is a $L_{\infty}$-stable local basis for $\mathcal{S}$, see e.g. [14]. A standard argument shows that this basis is $L_{p}$-stable after an appropriate renorming. Thus, for any $1 \leq p \leq \infty$ there are absolute constants $K_{1}, K_{2}>0$ such that for any coefficient vectors $\alpha=\left(\alpha_{\eta}\right)_{\eta \in \mathcal{M}}$,

$$
K_{1}\|\alpha\|_{p} \leq\left\|\sum_{\eta \in \mathcal{M}} \alpha_{\eta} h^{-2 / p} B_{\eta}\right\|_{L_{p}(\Omega)} \leq K_{2}\|\alpha\|_{p}
$$

where $h=1 / n$.

Let $\mathcal{T}$ be the subset of $\triangle$ defined in Section 2.4. As an intermediate approximation tool needed in the proof of our error bounds, we use the following quasi-interpolation operator $\hat{Q}: L_{1}(\Omega) \rightarrow \mathcal{S}$. Given $f \in L_{1}(\Omega)$ and $T \in \mathcal{T}$, let $\hat{p}_{T}$ denote the averaged Taylor polynomial of degree $q$ for $f$ over the largest disk contained in $T$ (cf. e.g. [31]), where $q=3$ if $\mathcal{S}=\mathcal{S}_{3}^{1}(\triangle), q=6$ if $\mathcal{S}=\mathcal{S S}_{6}^{2}(\triangle)$, and $q=5$ if $\mathcal{S}=\mathcal{R S}_{6}^{2}(\triangle)$. We set

$$
\hat{Q} f=\sum_{T \in \mathcal{T}} \sum_{\eta \in \mathcal{M}_{T}} c_{\eta}\left(\hat{p}_{T}\right) B_{\eta}
$$

The operator $\hat{Q}$ satisfies the following error bounds. 
Lemma 5.1. If $f$ belongs to the Sobolev space $W_{p}^{q+1}(\Omega)$, for some $1 \leq p \leq \infty$, then

$$
\|f-\hat{Q} f\|_{L_{p}(\Omega)} \leq K_{3} h^{q+1}|f|_{W_{p}^{q+1}(\Omega)},
$$

where $K_{3}$ is an absolute constant.

Proof: The lemma follows from Theorem 5.1 of [31] since all its hypotheses are satisfied due to Lemmas 4.1, 4.2, and 4.5-4.8, and the definitions of $\mathcal{S}$ and $\hat{Q}$. In particular, by (2.12) we have $\mathcal{P}_{q} \subset \mathcal{S}$, which ensures the polynomial reproduction property of $\hat{Q}$.

We denote by $Q_{1}, S Q_{2}$ and $R Q_{2}$ the approximation operators defined by (2.13) using our constructions for the spaces $\mathcal{S}_{3}^{1}(\triangle), \mathcal{S S}_{6}^{2}(\triangle)$ and $\mathcal{R S}_{6}^{2}(\triangle)$, respectively. The local polynomial approximations $p_{T}$ in (2.13) are determined as explained in Section 2.5. More precisely, for each $T \in \mathcal{T}$, let $\Omega_{T}$ be a subdomain of $\Omega$ such that $T \subset \Omega_{T}$. (Most often $\Omega_{T}$ just overlaps $T$ and possibly several neighboring triangles. See Section 6 for a description of how $\Omega_{T}$ is determined in our current implementation.) Let $p_{T}$ be the local polynomial approximation to the scattered data

$$
f(\xi), \quad \xi \in \Xi_{T}:=\Xi \cap \Omega_{T},
$$

obtained by the algorithm of Section 2.5, i.e. the degree $q_{T}$ of $p_{T}$ is adapted to $\Xi_{T}$, where we take 3,6 and 5 as a starting degree $q_{0}$ for the operators $Q_{1}, S Q_{2}$ and $R Q_{2}$, respectively. The coefficients of $p_{T}$ in the BB-representation (2.15) are computed by minimizing the error

$$
\sum_{\xi \in \Xi_{T}}\left(\sum_{i+j+k=q_{T}} c_{i j k} B_{i j k}^{q_{T}}(\xi)-f(\xi)\right)^{2} .
$$

Let, furthermore, $Q_{1}^{a v}, S Q_{2}^{a v}$ and $R Q_{2}^{a v}$ be the corresponding averaged operators (2.14).

The main results of this section are contained in the next two theorems giving estimates of the error of our approximation operators.

Theorem 5.2. Let $Q$ be one of the operators $Q_{1}, S Q_{2}$ or $R Q_{2}$, and let

$$
q:= \begin{cases}3, & \text { if } Q=Q_{1} \\ 6, & \text { if } Q=S Q_{2} \\ 5, & \text { if } Q=R Q_{2}\end{cases}
$$

If $f \in W_{p}^{q+1}(\Omega)$, for some $1 \leq p \leq \infty$, then

$$
\begin{aligned}
& \|f-Q f\|_{L_{p}(\Omega)} \leq K_{4} h^{q+1}|f|_{W_{p}^{q+1}(\Omega)}+K_{5}\left(\sum_{T \in \mathcal{T}}\left\|f-p_{T}\right\|_{L_{p}(T)}^{p}\right)^{1 / p}, \text { if } p<\infty, \\
& \|f-Q f\|_{L_{\infty}(\Omega)} \leq K_{6} h^{q+1}|f|_{W_{\infty}^{q+1}(\Omega)}+K_{7} \max _{T \in \mathcal{T}}\left\|f-p_{T}\right\|_{L_{\infty}(T)},
\end{aligned}
$$


where $K_{4}-K_{7}$ are absolute constants.

Proof: We give details of the proof only for $1 \leq p<\infty$ since the case $p=\infty$ is similar and simpler. In view of Lemma 5.1 it suffices to show that

$$
\|\hat{Q} f-Q f\|_{L_{p}(\Omega)} \leq K_{8} h^{q+1}|f|_{W_{p}^{q+1}(\Omega)}+K_{5}\left(\sum_{T \in \mathcal{T}}\left\|f-p_{T}\right\|_{L_{p}(T)}^{p}\right)^{1 / p}
$$

for some absolute constants $K_{5}, K_{8}$. We have

$$
\hat{Q} f-Q f=\sum_{T \in \mathcal{T}} \sum_{\eta \in \mathcal{M}_{T}} c_{\eta}\left(\hat{p}_{T}-p_{T}\right) B_{\eta}
$$

Then, by (5.2),

$$
\|\hat{Q} f-Q f\|_{L_{p}(\Omega)} \leq K_{2}\left(\sum_{T \in \mathcal{T}} \sum_{\eta \in \mathcal{M}_{T}}\left|h^{2 / p} c_{\eta}\left(\hat{p}_{T}-p_{T}\right)\right|^{p}\right)^{1 / p} .
$$

By the well known stability of the Bernstein-Bézier basis, see e.g. Lemma 4.1 in [31], there is an absolute constant $K_{9}$ such that

$$
\left(\sum_{\eta \in \mathcal{M}_{T}}\left|c_{\eta}\left(\hat{p}_{T}-p_{T}\right)\right|^{p}\right)^{1 / p} \leq K_{9} h^{-2 / p}\left\|\hat{p}_{T}-p_{T}\right\|_{L_{p}(T)}
$$

Therefore, by the local approximation properties of the averaged Taylor polynomial,

$$
\begin{aligned}
\|\hat{Q} f-Q f\|_{L_{p}(\Omega)} & \leq K_{2} K_{9}\left(\sum_{T \in \mathcal{T}}\left\|\hat{p}_{T}-p_{T}\right\|_{L_{p}(T)}^{p}\right)^{1 / p} \\
\leq & K_{2} K_{9}\left[\left(\sum_{T \in \mathcal{T}}\left\|f-\hat{p}_{T}\right\|_{L_{p}(T)}^{p}\right)^{1 / p}+\left(\sum_{T \in \mathcal{T}}\left\|f-p_{T}\right\|_{L_{p}(T)}^{p}\right)^{1 / p}\right] \\
\leq & K_{10} h^{q+1}\left(\sum_{T \in \mathcal{T}}|f|_{W_{p}^{q+1}(T)}^{p}\right)^{1 / p}+K_{2} K_{9}\left(\sum_{T \in \mathcal{T}}\left\|f-p_{T}\right\|_{L_{p}(T)}^{p}\right)^{1 / p},
\end{aligned}
$$

with some absolute constant $K_{10}$, which implies (5.4).

It is clear that similar bounds are valid for each operator $Q^{[i]}, i=1, \ldots, 8$, involved in the definition of the corresponding averaged operator $Q^{a v}$ defined by (2.14). Therefore, the estimate for $\left\|f-Q^{a v} f\right\|_{L_{p}(\Omega)}$ presented in the following theorem easily follows from the inequality

$$
\left\|f-Q^{a v} f\right\|_{L_{p}(\Omega)} \leq \frac{1}{8} \sum_{i=1}^{8}\left\|f-Q^{[i]} f\right\|_{L_{p}(\Omega)}
$$


and the fact that

$$
\triangle=\bigcup_{i=1}^{8} \mathcal{T}^{[i]} .
$$

Theorem 5.3. Let $Q^{a v}$ be one of the operators $Q_{1}^{a v}, S Q_{2}^{a v}$ or $R Q_{2}^{a v}$, and let $q$ be defined as in (5.3) for the corresponding non-averaged operators. If $f \in W_{p}^{q+1}(\Omega)$, for some $1 \leq p \leq \infty$, then

$$
\begin{aligned}
& \left\|f-Q^{a v} f\right\|_{L_{p}(\Omega)} \leq K_{11} h^{q+1}|f|_{W_{p}^{q+1}(\Omega)}+K_{12}\left(\sum_{T \in \triangle}\left\|f-p_{T}\right\|_{L_{p}(T)}^{p}\right)^{1 / p}, \text { if } p<\infty, \\
& \left\|f-Q^{a v} f\right\|_{L_{\infty}(\Omega)} \leq K_{13} h^{q+1}|f|_{W_{\infty}^{q+1}(\Omega)}+K_{14} \max _{T \in \triangle}\left\|f-p_{T}\right\|_{L_{\infty}(T)},
\end{aligned}
$$

where $K_{11}-K_{14}$ are absolute constants.

Note that the approximation error $\left\|f-p_{T}\right\|_{L_{p}(T)}$ of the local polynomials $p_{T}$ involved in the estimates of Theorems 5.2 and 5.3 depends on the distribution of the local data $\Xi_{T}$ as well as a number of parameters such as the degree $q_{T}$ of $p_{T}$, the size of $\Omega_{T}$ relative to $T$, the number of points in $\Xi_{T}$, and the tolerance value $\kappa$ used in the algorithm of Section 2.5. For instance, by (2.8) in [9] it follows that

$$
\left\|f-p_{T}\right\|_{L_{\infty}(T)} \leq K_{15} C_{T} \kappa \sqrt{\# \Xi_{T}} \operatorname{diam}\left(\Omega_{T}\right)^{q_{T}+1}|f|_{W_{\infty}^{q_{T}+1}\left(\Omega_{T}\right)},
$$

where $K_{15}$ is a constant depending only on $q_{T}$, and

$$
C_{T}=\sup _{\|\alpha\|_{2} \leq 1}\left\|\sum_{i+j+k=q_{T}} \alpha_{i j k}^{q_{T}} B_{i j k}^{q_{T}}\right\|_{L_{\infty}\left(\Omega_{T}\right)}
$$

where $B_{i j k}^{q_{T}}$ are the Bernstein polynomials of degree $q_{T}$ with respect to the triangle $T$, and $\alpha=\left(\alpha_{i j k}^{q_{T}}\right)_{i+j+k=q_{T}}$.

Remark 5.4. Our local polynomial approximations have optimal approximation order $\mathcal{O}\left(h^{q+1}\right)$, with $q$ defined in (5.3), if the data is dense enough. More precisely, if the (local) fill distance

$$
h\left(\Xi_{T}, \Omega_{T}\right):=\max _{x \in \Omega_{T}} \min _{\xi \in \Xi_{T}}\|x-\xi\|_{2}
$$

is small enough, e.g. if $h\left(\Xi_{T}, \Omega_{T}\right)<0.11 h / q^{2}$ in the case when $\Omega_{T}$ is a circle of radius $h$, then the so called norming constant of $\mathcal{P}_{q}$ with respect to $\Xi_{T}$ is at least $1 / 2$ (see, e.g. [60]), which implies by Theorem 2.1 of [9] that $\sigma_{\min }^{-1}$ remains small, and therefore $q_{T}=q$ by the algorithm of Section 2.5 provided the tolerance value $\kappa$ is appropriately chosen. 


\section{$\S 6$. Numerical Examples}

We present here results of numerical tests on the approximation order, shape recovery, compression and denoising using standard test functions as well as tests with real world data. We begin with comments on computational aspects of the method and its current implementation.

\subsection{Computational Aspects}

Some important algorithmic details of the non-averaged $C^{1}$ method skipped in this paper can be found in [23]. These concern for instance the initial data sorting, local data thinning, computational aspects of discrete least squares polynomial approximation, usage of Bernstein-Bézier techniques for rendering, and boundary treatment where attaching auxiliary strips of squares turned out to be advantageous since it helped to simplify the implementation by avoiding the special consideration of the boundary.

In the current implementation, we apply the same principles to the $C^{2}$ method and to the averaged methods. In order to compute the local least squares polynomials $p_{T}$, for each $T \in \mathcal{T}$, we initially choose the local domain $\Omega_{T}$ overlapping a neighborhood of $T$ (see Section 2.5), to be the circle of radius $h$ centered at the barycenter of $T$. (Here $h=1 / n$ is the side of $T$.) However, if for some $T \in \mathcal{T}$, the number $m=\#\left(\Xi \cap \Omega_{T}\right)$ does not lie within a heuristically chosen interval $\left[M_{\min }, M_{\max }\right]$, we either increase the radius of the circle if $m<M_{\min }$, or we apply a grid-type thinning method (see [23] for details) to reduce the number of points in $\Xi_{T}=\Xi \cap \Omega_{T}$ if $m>M_{\max }$. Then, the adaptive algorithm of Section 2.5 is used. Note that the termination criterion $\sigma_{q, T}^{-1} \leq \kappa$ based on the explicit estimates of the approximation error of local least squares given in [9] has shown in our extensive tests a superior performance over the earlier criterion $\operatorname{cond}_{q, T}=\left\|M_{q, T}\right\|_{2} / \sigma_{q, T} \leq \kappa$ used in [23]. (Here, $M_{q, T}$ is the collocation matrix from (2.16).) Therefore, $\kappa$ has a slightly different meaning in this paper comparing to [23]. Although $M_{\max }$ is mainly needed to reduce the computational costs, $\kappa$ and $M_{\text {min }}$ are important $p a$ rameters of our method that allow to adjust it to various types of scattered data, similar to the role of parameters in the scattered data techniques based on different approachs (cf. e.g. $[36,56]$ ).

The above computations have a local nature, i.e., they are applied to small portions of the data concentrated in $\mathcal{O}(N)$ subdomains $\Omega_{T}, T \in \mathcal{T}$, where $N$ is the total number of points in $\Xi$. (We assume that the dimension $D$ of the spline space is chosen of the same order of magnitude $\mathcal{O}(N)$.) Since the size of each matrix (2.16) is at most $10 \times M_{\max }$ (respectively, $28 \times M_{\max }$ ) in the $C^{1}$ (respectively, $C^{2}$ ) case, the computation of all local least squares polynomials requires $\mathcal{O}(N)$ computational time and memory. (Note that each polynomial is computed independently, such that this most expensive step of the algorithm can be easily parallelized to take advantage of a multiprocessor computer.) To find the points located in $\Omega_{T}$ efficiently, the data is decomposed into $\mathcal{O}(N)$ grid cells at a pre-processing stage. This approach guarantees the linear overall complexity of the algorithm (i.e., $\mathcal{O}(N)$ 
time and memory requirements) at least in the case when the fill distance $h(\Xi, \Omega)$ satisfies

$$
h(\Xi, \Omega):=\max _{x \in \Omega} \min _{\xi \in \Xi}\|x-\xi\|_{2}=\mathcal{O}(1 / \sqrt{N}) .
$$

Without this assumption the complexity may rise to $\mathcal{O}(N \log N)$ if for a substantial number of $\Omega_{T}$ many consecutive extension steps are needed to find at least $M_{\min }$ local data points (compare [56]). Our tests with random and real world data confirm the linear complexity of the algorithm.

Since we compute the complete BB-representation (2.1) of the approximating spline $s$, the subsequent evaluation of $s$, its derivatives and normals can be done efficiently by using the well known de Casteljau algorithm (cf. e.g. [16]), with the computational cost $\mathcal{O}(M)$ for $M$ evaluations. Typical CPU time for the evaluation of the spline at 1,000,000 points is about $1.4 \mathrm{sec}$ in the $C^{1}$ case, and $3.1 \mathrm{sec}$ in the $C^{2}$ case.

In what follows we denote our approximation operators by $Q_{1}, S Q_{2}, R Q_{2}$, $Q_{1}^{a v}, S Q_{2}^{a v}$ and $R Q_{2}^{a v}$, as in Section 5. We concentrate in Sections 6.3-6.6 on tests with $Q_{1}^{a v}$ and $R Q_{2}^{a v}$ since the methods based on $\mathcal{R S}_{6}^{2}(\triangle)$ have generally shown a better performance then those based on $\mathcal{S S}_{6}^{2}(\triangle)$ (except the approximation order tests), and since the averaged methods generally produce more pleasant surfaces and smaller errors for the same data sets.

The tests presented below were made on a Sun Ultra 60 workstation with a $450 \mathrm{MHz}$ processor. The surfaces were visualized with Matlab using evaluations of the splines on a fine grid $(101 \times 101$ grid for the test functions of Sections 6.3-6.5, $301 \times 361$ grid for the Glacier, $601 \times 601$ grid for the Black Forest and $601 \times 1630$ grid for the Rotterdam Port data set.) For the non-square domains (i.e. for Glacier and Rotterdam Port data sets) we defined the triangulation by subdividing the bounding box of $\Xi$ into $n m$ rectangles of almost square shape using $n-1$ vertical and $m-1$ horizontal lines. The dimension formulas for the spline spaces in this case read as follows:

$$
\begin{aligned}
& \operatorname{dim} \mathcal{S}_{3}^{1}(\triangle)=5 n m+4(n+m)+3 \\
& \operatorname{dim} \mathcal{S}_{6}^{2}(\triangle)=19 n m+12(n+m)+6, \\
& \operatorname{dim} \mathcal{S S}_{6}^{2}(\triangle)=\operatorname{dim} \mathcal{R S}_{6}^{2}(\triangle)=14 n m+12(n+m)+6
\end{aligned}
$$

We use the following notations in the tables of Sections 6.2-6.6: $n, m$ denote the triangulation parameters as above ( $m$ is omitted if it is equal to $n$ ), $D$ is the dimension of the spline space used in the test, $N$ is the number of data points, $\kappa, M_{\min }$ and $M_{\max }$ are the parameters of the method, as described above, and $t$ is the computational time in seconds, with the exception of Tab. 2, where the time is given in minutes. Finally, max, mean and $r m s$ denote the maximum error, the average error, and the root mean square error, respectively. The errors are calculated against the exact values of the test function on a dense grid containing $\approx 9 N$ points for the tests of Section 6.2 , and on the $101 \times 101$ grid for the tests of Sections 6.3-6.5. For the real world data sets of Section 6.6, max, mean and rms are computed with respect to the data. 


\subsection{Approximation Order}

To test the approximation power of the method, we performed extensive numerical experiments with the well known Franke exponential test function from [19-21],

$$
\begin{aligned}
f(x, y)= & \frac{3}{4} \exp \left[-\frac{(9 x-2)^{2}+(9 y-2)^{2}}{4}\right]+\frac{3}{4} \exp \left[-\frac{(9 x+1)^{2}}{49}-\frac{(9 y+1)}{10}\right] \\
& +\frac{1}{2} \exp \left[-\frac{(9 x-7)^{2}+(9 y-3)^{2}}{4}\right]-\frac{1}{5} \exp \left[-(9 x-4)^{2}-(9 y-7)^{2}\right] .
\end{aligned}
$$

(The shape of this function is shown in Fig. 12a.) For $N$ in the range between 1,000 and 100,000 , we generated $N$ random data points in $[0,1]^{2}$. Then we evaluated the test function at these points and computed spline approximations using our operators $Q_{1}, S Q_{2}, Q_{1}^{a v}$ and $S Q_{2}^{a v}$, with the grid size chosen such that the dimension of the respective spline space $\mathcal{S}_{3}^{1}(\triangle)$ or $\mathcal{S S}_{6}^{2}(\triangle)$ approximately equals $N$. Tab. 1 gives averaged values (using 40 data sets for each $N$ ) for the maximal approximation error max and computational time $t$ in seconds. We have chosen $M_{\text {min }}=11$ for the $C^{1}$ method, and $M_{\min }=29$ for the $C^{2}$ method, such that almost all local polynomials (at least $99 \%$ in each test) are of the highest degree. The results clearly indicate the linear grows of the computational costs as well as almost optimal approximation order. (Recall that the best possible approximation order for piecewise polynomials of degree $d$ is $h^{d+1}$, or $N^{-(d+1) / 2}$.) Similar tests with the operators $R Q_{2}$, and $R Q_{2}^{a v}$ (not shown in the table) also confirm their theoretical behavior.

\begin{tabular}{|c|c|c|c|c|c|c|c|c|}
\hline \multirow{2}{*}{$N$} & \multicolumn{2}{|c|}{$Q_{1}$} & \multicolumn{2}{c|}{$Q_{1}^{a v}$} & \multicolumn{2}{c|}{$S Q_{2}$} & \multicolumn{2}{c|}{$S Q_{2}^{a v}$} \\
\cline { 2 - 9 } & $\max$ & $t$ & $\max$ & $t$ & $\max$ & $t$ & $\max$ & $t$ \\
\hline $10^{3}$ & $8.6 \cdot 10^{-3}$ & 0.05 & $3.1 \cdot 10^{-3}$ & 0.40 & $1.7 \cdot 10^{-2}$ & 0.26 & $4.8 \cdot 10^{-3}$ & 2.1 \\
$2 \cdot 10^{3}$ & $2.9 \cdot 10^{-3}$ & 0.09 & $1.0 \cdot 10^{-3}$ & 0.72 & $2.2 \cdot 10^{-3}$ & 0.47 & $5.2 \cdot 10^{-4}$ & 3.8 \\
$5 \cdot 10^{3}$ & $7.1 \cdot 10^{-4}$ & 0.20 & $2.8 \cdot 10^{-4}$ & 1.6 & $1.4 \cdot 10^{-4}$ & 1.1 & $2.7 \cdot 10^{-5}$ & 8.7 \\
$10^{4}$ & $2.4 \cdot 10^{-4}$ & 0.39 & $8.0 \cdot 10^{-5}$ & 3.1 & $1.2 \cdot 10^{-5}$ & 2.0 & $2.7 \cdot 10^{-6}$ & 16 \\
$2 \cdot 10^{4}$ & $7.6 \cdot 10^{-5}$ & 0.79 & $2.5 \cdot 10^{-5}$ & 6.4 & $1.2 \cdot 10^{-6}$ & 3.9 & $3.3 \cdot 10^{-7}$ & 31 \\
$5 \cdot 10^{4}$ & $2.1 \cdot 10^{-5}$ & 2.1 & $6.2 \cdot 10^{-6}$ & 17 & $6.0 \cdot 10^{-8}$ & 9.7 & $1.6 \cdot 10^{-8}$ & 78 \\
$10^{5}$ & $7.9 \cdot 10^{-6}$ & 4.2 & $2.6 \cdot 10^{-6}$ & 34 & $6.7 \cdot 10^{-9}$ & 19 & $1.9 \cdot 10^{-9}$ & 155 \\
\hline
\end{tabular}

Tab. 1. Approximation order.

In addition, Tab. 2 gives the results of our tests with some very large randomly generated data sets (up to 13 millions points). Here and below $D$ indicates the dimension of the spline space which is not always the same as the number of data points $N$. In the cases when $D$ is much smaller than $N$, we have taken $M_{\max }=100$ for the $C^{1}$ methods, and 300 for the $C^{2}$ methods.

Note that the data sets obtained in this way are very "smooth" and therefore the choice of parameters $\kappa$ and $M_{\text {min }}$ does not play such an important role as in the case of the real world data. 


\begin{tabular}{|c|c|c|c|c|c|c|c|}
\hline$N$ & $n$ & $D$ & method & max & mean & $r m s$ & $t(\min )$ \\
\hline $1.3 \cdot 10^{7}$ & 512 & $1.3 \cdot 10^{6}$ & $Q_{1}$ & $2.3 \cdot 10^{-9}$ & $9.6 \cdot 10^{-11}$ & $1.7 \cdot 10^{-10}$ & 3.5 \\
$1.3 \cdot 10^{7}$ & 512 & $1.3 \cdot 10^{6}$ & $Q_{1}^{a v}$ & $5.6 \cdot 10^{-10}$ & $4.1 \cdot 10^{-11}$ & $6.3 \cdot 10^{-11}$ & 30 \\
$5 \cdot 10^{6}$ & 1000 & $5 \cdot 10^{6}$ & $Q_{1}$ & $1.1 \cdot 10^{-7}$ & $1.6 \cdot 10^{-10}$ & $3.0 \cdot 10^{-10}$ & 3.6 \\
$5 \cdot 10^{6}$ & 1000 & $5 \cdot 10^{6}$ & $Q_{1}^{a v}$ & $4.6 \cdot 10^{-8}$ & $1.5 \cdot 10^{-10}$ & $2.4 \cdot 10^{-10}$ & 29 \\
$2.4 \cdot 10^{6}$ & 128 & $2.3 \cdot 10^{5}$ & $S Q_{2}$ & $3.0 \cdot 10^{-10}$ & $3.3 \cdot 10^{-12}$ & $5.7 \cdot 10^{-12}$ & 4 \\
$2.4 \cdot 10^{6}$ & 128 & $2.3 \cdot 10^{5}$ & $R Q_{2}$ & $3.6 \cdot 10^{-10}$ & $5.4 \cdot 10^{-12}$ & $1.4 \cdot 10^{-11}$ & 4 \\
\hline
\end{tabular}

Tab. 2. Tests with large data sets.

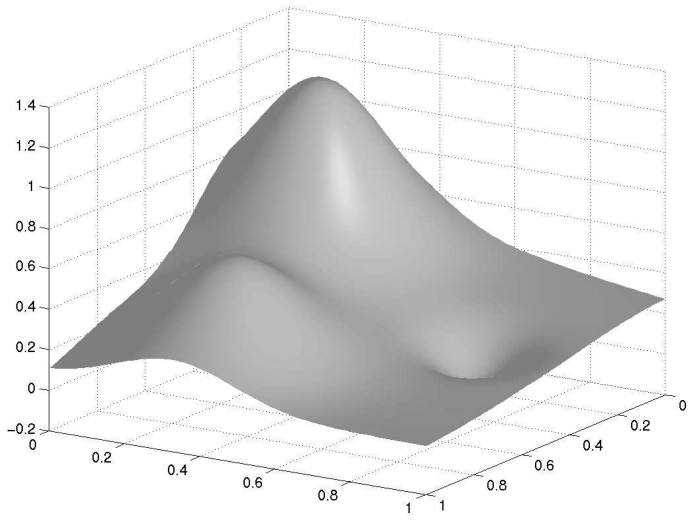

(a)

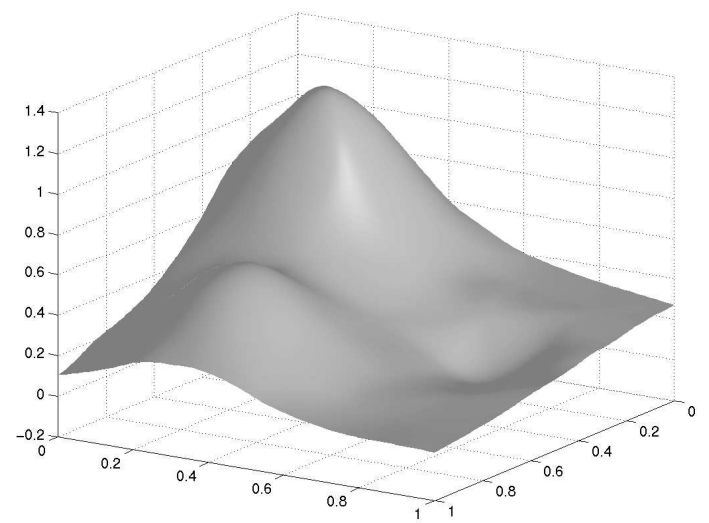

(c)

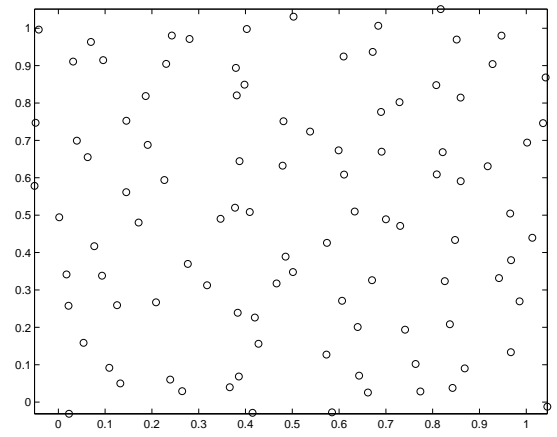

(b)

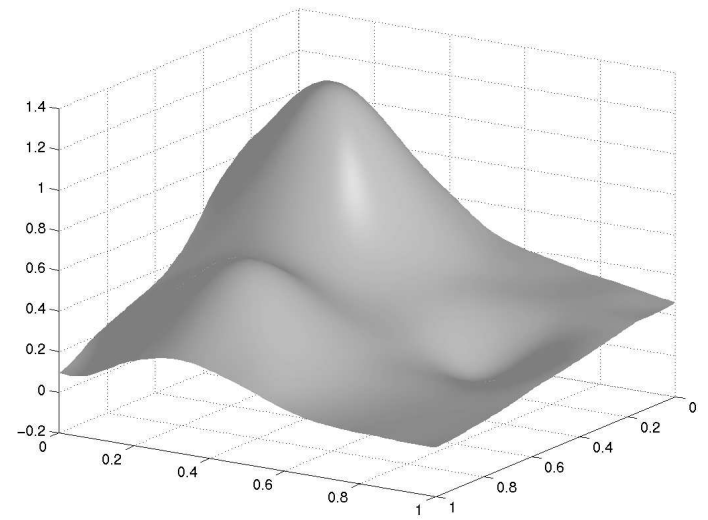

(d)

Fig. 12. Shape recovery tests: (a) Franke test function. (b) Locations of 100 points of ds3. (c) $C^{1}$ spline. (d) $C^{2}$ spline.

\subsection{Shape Recovery}

The next test problem we consider is the recovery of the shape of a test function from a small number of scattered data points. We again use the Franke test function (Fig. 12a) and a standard set of 100 points sampled at the location shown in Fig. 12b. These points (available from [21] as ds3) were used in [19] to test various scattered data fitting methods. We present in Fig. 12c and $12 \mathrm{~d}$ the shapes recov- 
ered using our method. Tab. 3 shows the parameters used and the errors of the spline approximations calculated on a uniform $101 \times 101$ grid. Note that the values for $\kappa$ and $M_{\min }$ were found in tests with random point sets of the same size 100, and that we start here the algorithm of Section 2.5 for $R Q_{2}^{a v}$ with degree 6 (see also Tab. 7).

\begin{tabular}{|c|c|c|c|c|c|c|c|c|}
\hline fig & method & $n$ & $D$ & $\kappa$ & $M_{\min }$ & $\max$ & mean & rms \\
\hline $12 \mathrm{c}$ & $Q_{1}^{a v}$ & 6 & 231 & 32 & 3 & 0.043 & 0.0070 & 0.0101 \\
$12 \mathrm{~d}$ & $R Q_{2}^{a v}$ & 5 & 476 & 32 & 16 & 0.038 & 0.0052 & 0.0076 \\
\hline
\end{tabular}

Tab. 3. Shape recovery tests.

\subsection{Compression}

A different problem is to represent a shape with as few degrees of freedom as possible. In contrast to the previous subsection, we assume that a sufficient amount of information is available, i.e., the data set is dense. If this is not the case, then first the shape recovery can be applied as above, and, subsequently, the dense evaluations of the resulting spline surface can be used as input for a second step aimed at compression.

As a test function we choose the "curved valleys" benchmark function

$$
f(x, y)=0.5 y \cos ^{4}\left(4\left(x^{2}+y-1\right)\right),
$$

whose shape is shown in Fig. 13a (cf. [22,39,40]). Using 10,201 values of this function on a regular grid, we run our $C^{1}$ averaged method with spline dimensions 72,115 and 168 . The (nominal) values of 7 and $10^{7}$, respectively, are assigned to the parameters $M_{\min }$ and $\kappa$. Because of the high density of the data there are between 1000 and 4000 points in the local subdomains $\Omega_{T}$ for this tests. Therefore we also use them to demonstrate the reduction of the computational time achievable by the appropriate choice of the parameter $M_{\max }$. Since we take relatively high values for $M_{\max }$, the degrees of local polynomials remain 3 for all $\Omega_{T}$. Results are presented in Fig. 13 and Tab. 4. (The errors are evaluted on the same $101 \times 101$ grid.) Note that there is no visual difference in the appearance of the surfaces calculated with $M_{\max }=300$ and $M_{\max }=10000$. An important observation is that due to the regularity of the four directional mesh, the number of real numbers needed to store a spline surface is equal to the dimension $D$ of the space if the position of the (rectangular) domain is known.

\begin{tabular}{|c|c|c|c|c|c|c|}
\hline fig & $n$ & $D$ & max & mean & rms & $t$ \\
\hline $13 \mathrm{~b}$ & 3 & 72 & 0.117 & 0.0298 & 0.0401 & 0.48 \\
$13 \mathrm{c}$ & 4 & 115 & 0.105 & 0.0140 & 0.0199 & 0.48 \\
$13 \mathrm{~d}$ & 5 & 168 & 0.094 & 0.0101 & 0.0150 & 0.72 \\
\hline
\end{tabular}

(a)

\begin{tabular}{|c|c|c|c|}
\hline max & mean & rms & $t$ \\
\hline 0.114 & 0.0278 & 0.0378 & 3.04 \\
0.104 & 0.0132 & 0.0189 & 3.12 \\
0.091 & 0.0095 & 0.0143 & 3.12 \\
\hline
\end{tabular}

(b)

Tab. 4. Compression tests: (a) $M_{\max }=300$, (b) $M_{\max }=10000$. 


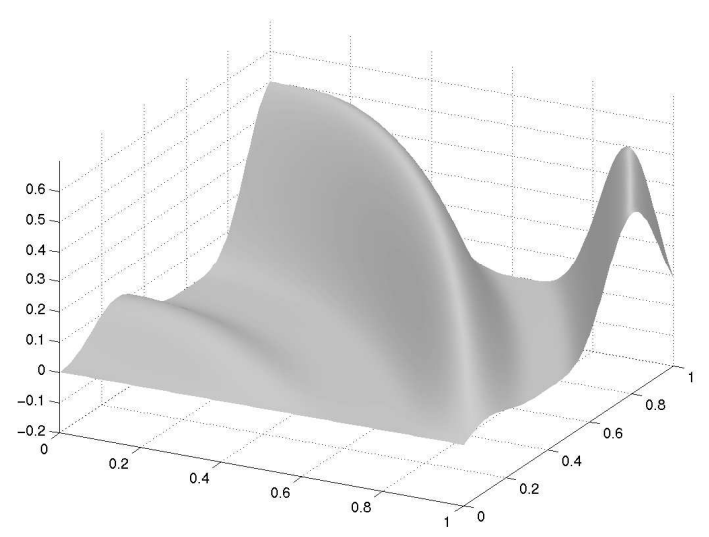

(a)

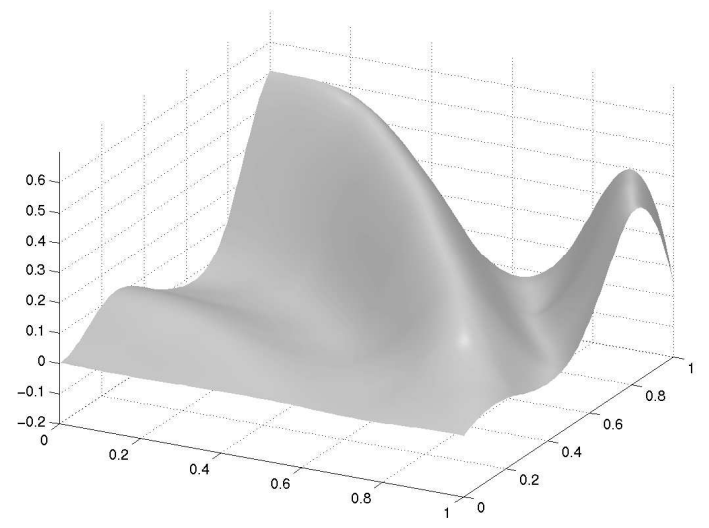

(c)

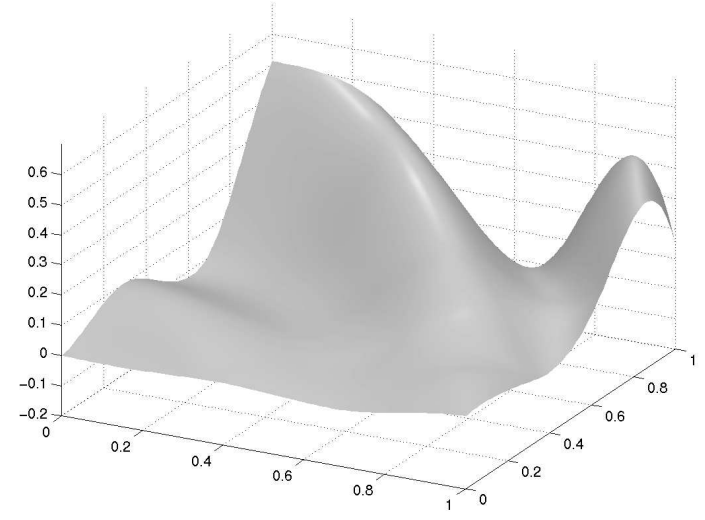

(b)

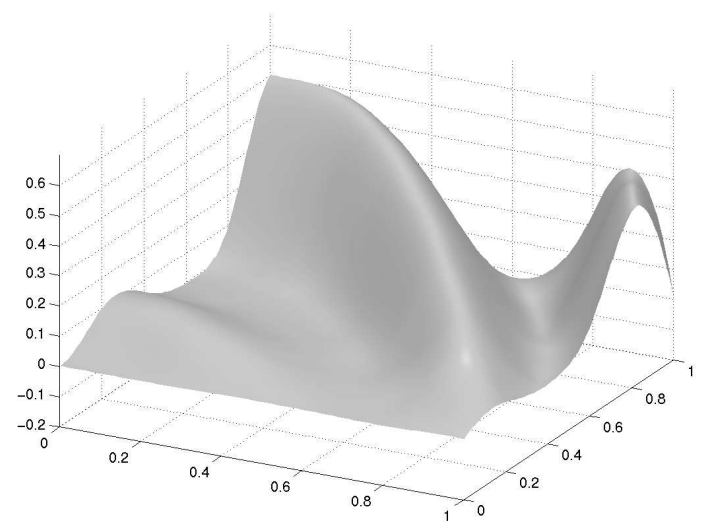

(d)

Fig. 13. Compression tests: (a) "Curved valleys" test function. (b)-(d) $C^{1}$ spline approximation with 72,115 , and 168 parameters, respectively (all computed with $M_{\max }=300$ ).

\subsection{Denoising}

To test the performance of the method in the case of noisy data, we used two data sets contaminated with normally distributed random errors with standard deviation 0.05 . The first of them is obtained by adding the random errors to the evaluations of the Franke test function (Fig. 12a) on a $101 \times 101$ grid. We use the operator $Q_{1}^{a v}$. The resulting noisy surface is visualized in Fig. 14a using Matlab mesh command, while the shape recovered by our method is presented in Fig. 14b. The computational time is 2.88 seconds. (Note that we use $M_{\max }=600$.) The rms error in Tab. 5 shows the reduction of the noise with a factor more than 9 . The second data set is obtained in the same way from the values of the Franke test function at 500 locations shown in Fig. 14c. It was used in [38] to test a scattered data fitting algorithm based on thin plate splines and is available from [21] as vde500. Our results are given in Fig. 14d and Tab. 5. In both cases the errors in Tab. 5 are computed on a $101 \times 101$ grid against the exact values of the test function. Note that choosing $\kappa$ as small as 1 was very essential for the performance 


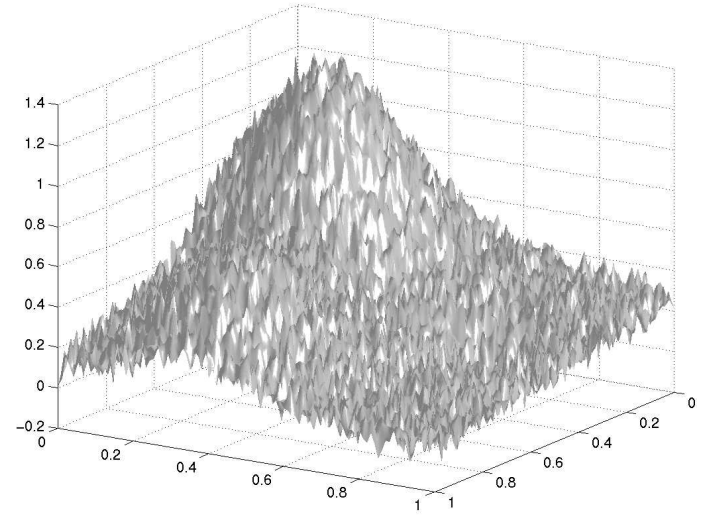

(a)

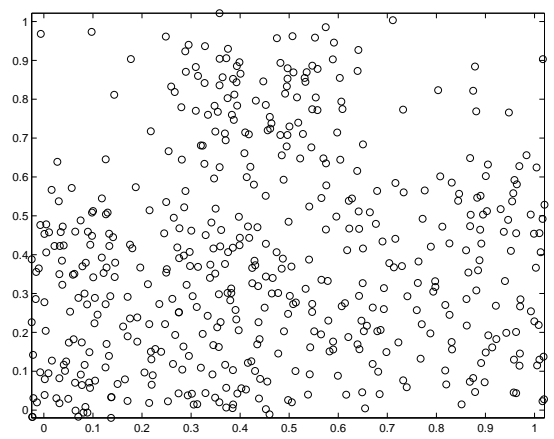

(c)

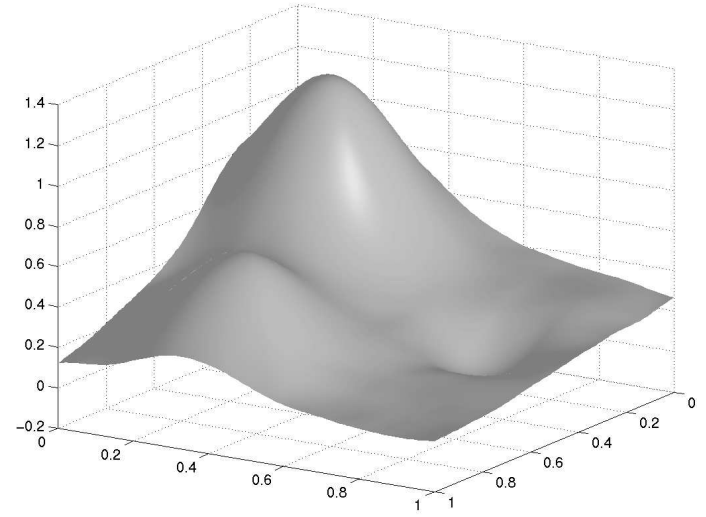

(b)

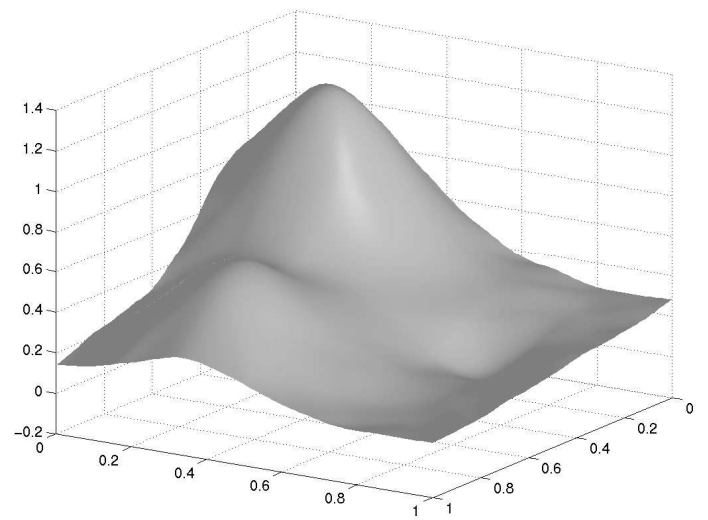

(d)

Fig. 14. Tests with noisy data: (a) Franke test function contaminated on a $101 \times 101$ grid with normal noise of standard deviation 0.05 . (b) $C^{1}$ spline reconstruction using this data set. (c) Locations of 500 points of vde500. (d) $C^{1}$ spline reconstruction using 500 contaminated points.

\begin{tabular}{|c|c|c|c|c|c|c|c|c|}
\hline fig & method & $\mathrm{n}$ & $D$ & $\kappa$ & $M_{\min }$ & max & mean & rms \\
\hline $14 \mathrm{~b}$ & $Q_{1}^{a v}$ & 7 & 304 & 1 & 7 & 0.0260 & 0.0040 & 0.0052 \\
$14 \mathrm{~d}$ & $Q_{1}^{a v}$ & 6 & 231 & 1 & 1 & 0.0494 & 0.0145 & 0.0178 \\
\hline
\end{tabular}

Tab. 5. Tests with noisy data.

shown in these tests.

In addition, we repeated these tests using the same local polynomial approximations of degree not more than 3 , but applying the $C^{2}$ method based on the space $\mathcal{R S}_{6}^{2}(\triangle)$ in the second stage. The resulting surfaces do not look different from those in Fig. 14b and 14d, but the errors reduce to $\max =0.0201$, mean $=0.0039$, $r m s=0.0051$, for the first test, and $\max =0.0435$, mean $=0.0136, r m s=0.0169$, for the second test. Note that the computational time does not change since the second stage of the algorithm in both cases is much faster than the first stage. 


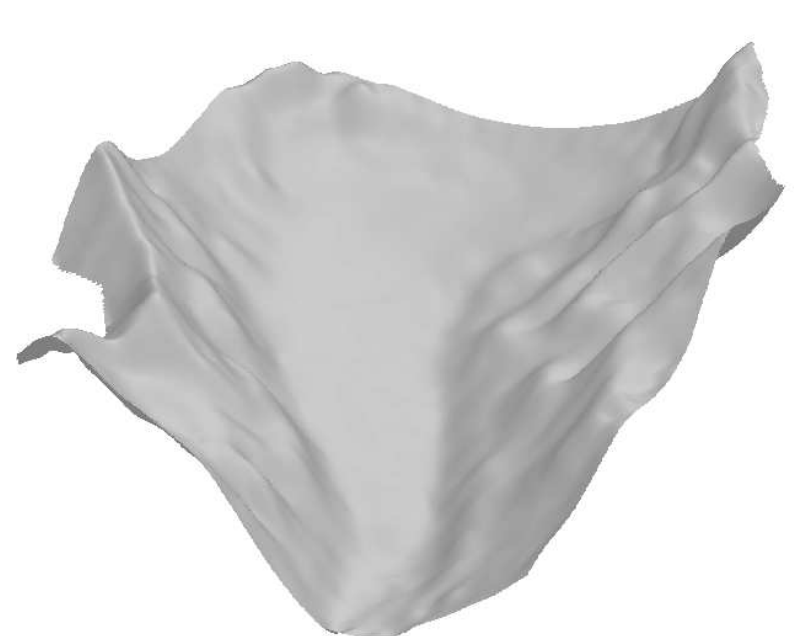

(a)

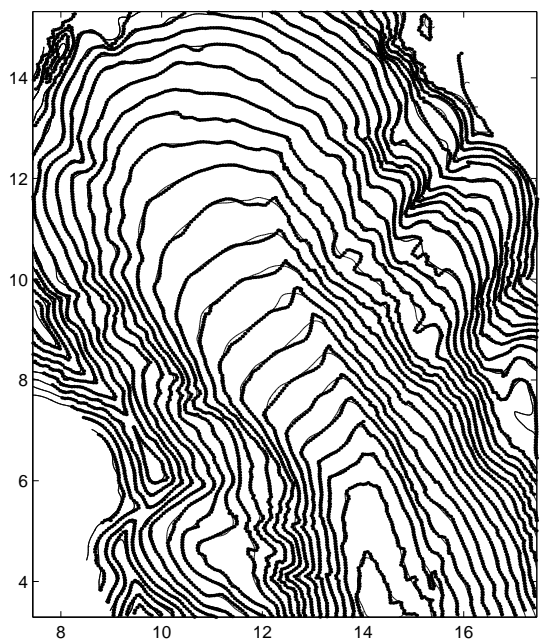

(b)

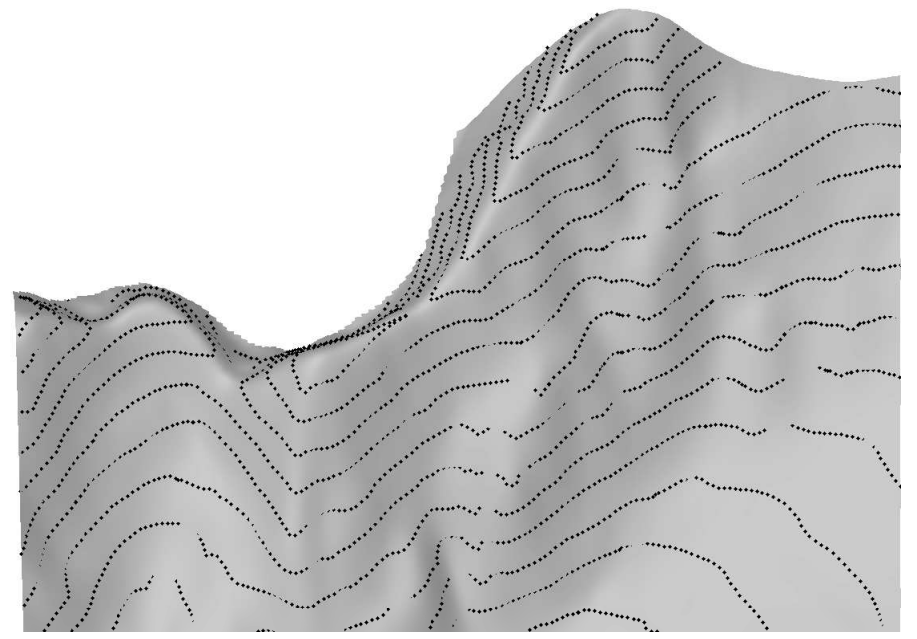

(c)

Fig. 15. $C^{2}$ spline approximation of glacier data: (a) General view. (b) Contour plot (the thick lines consist of the locations of the data points, while the thin lines are the contours of the spline at the same 44 hight levels). (c) Screenshot of the spline surface with the data points as dots.

\subsection{Real World Tests}

1. Glacier. As a first real world data set we use 8,345 points (available from [21] as vol87) representing 44 digitized height contours of a glacier (cf. $[38,56,60]$ ). The difference between the maximal and minimal heights is $800 \mathrm{~m}$. We approximate this data with a $C^{2}$ spline with 7,254 degrees of freedom using the operator $R Q_{2}^{a v}$ and parameters $n=20, m=24, \kappa=2, M_{\min }=60, M_{\max }=160$, starting the local stage with degree 5, as explained in Section 5. The spline surface is shown in Fig. 15. Note that the contour lines of the spline surface in Fig. 15b are almost everywhere covered with the original data points. However, in some places smoothing effects are visible. The errors at the data are $\max =18.66 \mathrm{~m}$, mean $=1.95 \mathrm{~m}$, and $r m s=2.78 \mathrm{~m}$. The computational time is 19 seconds. 


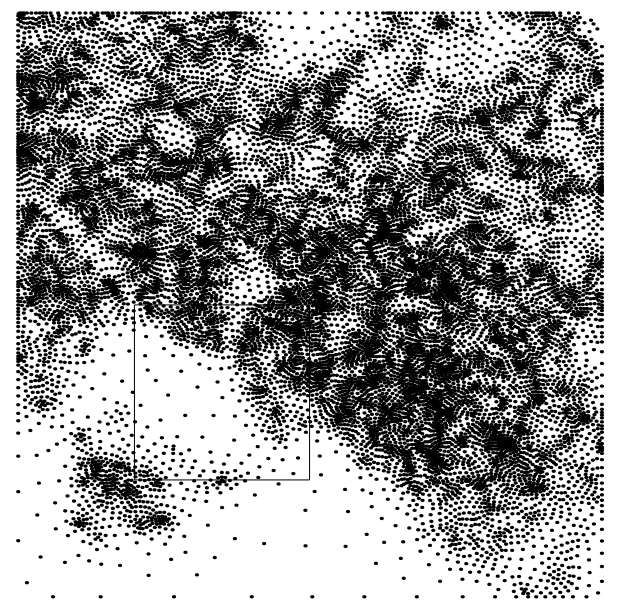

(a)

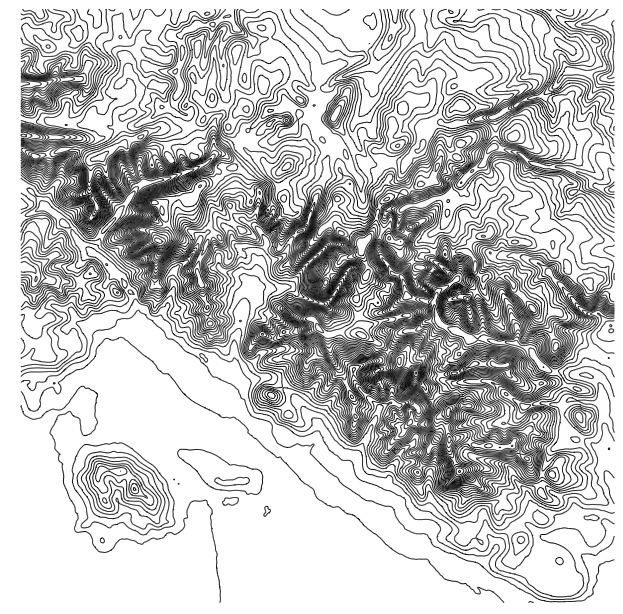

(b)

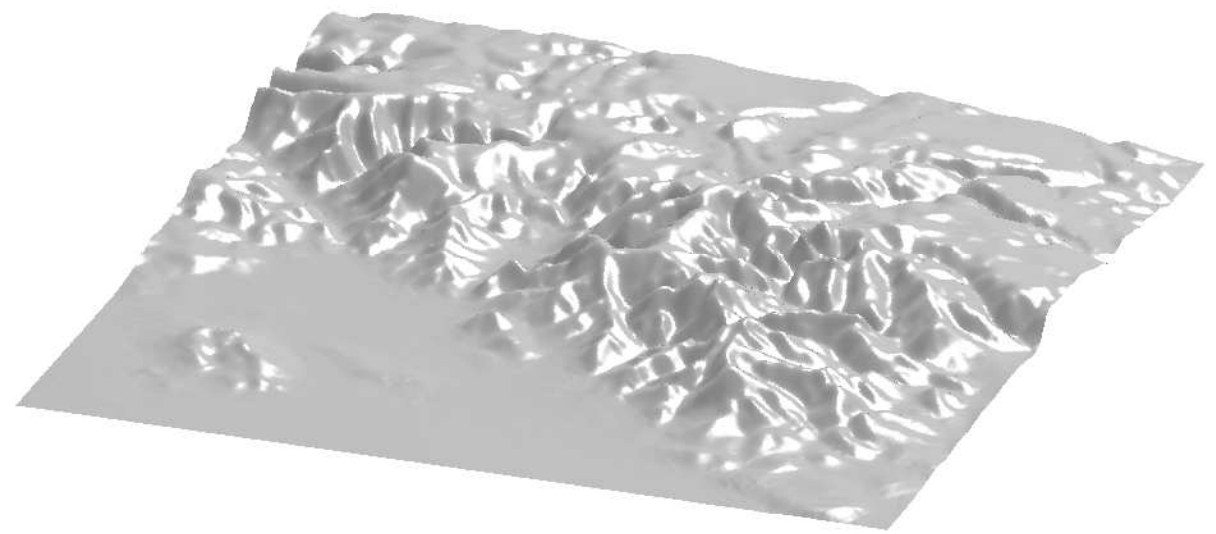

(c)

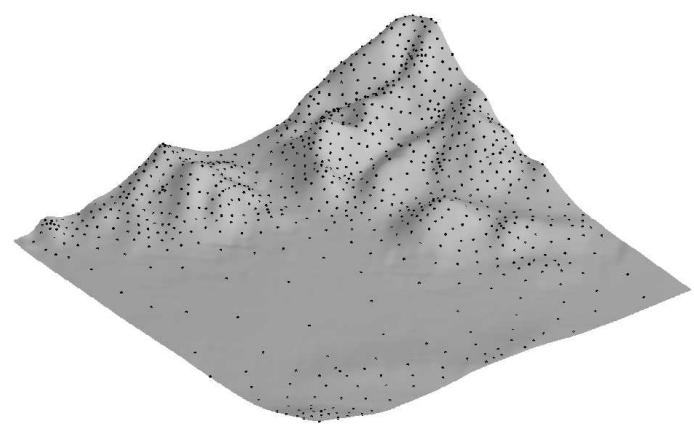

(d)

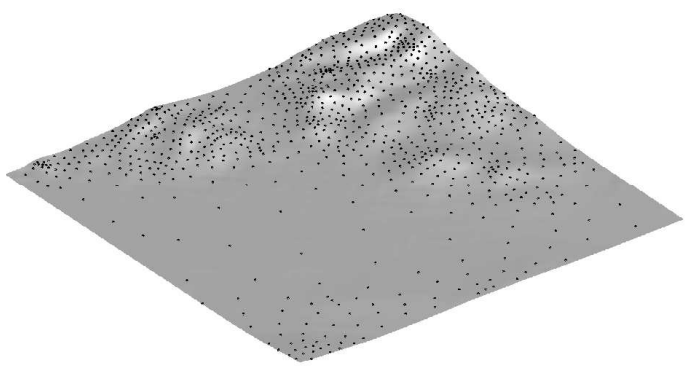

(e)

Fig. 16. $C^{2}$ spline approximation of black forest data: (a) Locations of the data points. (b) Contour lines of the approximation. (c) General view. (d) Spline surface in the area indicated with a box in (a) (the data points are shown as black dots). (e) The same with real proportions. 
2. Black Forest. Another data set consists of 15,885 points representing a terrain in the neighborhood of Freiburg, Germany, see Fig. 16. The difference between the heighest and the lowest point is $1,214 \mathrm{~m}$. (Note that the data are scaled in the horizontal plane with a factor $\approx 1 / 4$ to the real proportions, compare Fig. $16 \mathrm{~d}$ and e.) We again use the $C^{2}$ method $R Q_{2}^{a v}$ starting the local stage with degree 5. The number of degrees of freedom $(91,526)$ is chosen substantially higher than the number of points in order to achieve high approximation quality in the areas where the data set is dense. This, however, did not produce any substantial oscillations in the extremely sparse areas, see Fig. 16d. The values of parameters used to produce this spline surface: $n=m=80, \kappa=5, M_{\min }=3 . M_{\max }=100$. The errors at the data are $\max =30.56 \mathrm{~m}$, mean $=2.563 \mathrm{~m}$, and $\mathrm{rms}=3.567 \mathrm{~m}$. The computational time is 9.8 seconds.

3. Rotterdam Port. Finally, we apply our method $\left(Q_{1}^{a v}\right)$ to a set of 634,604 raw data points in an almost rectangular domain of size $367 \mathrm{~m}$ by $997 \mathrm{~m}$ produced by the high density multibeam echosounder for the purpose of dredge monitoring of the Rotterdam harbor. A typical distribution of the data locations is shown in Fig. 17a. The heights vary between $-27.62 \mathrm{~m}$ and $-5.26 \mathrm{~m}$ to the sea level. First, we apply our algorithm to the full (raw) dataset and compute the averaged $C^{1}$ spline with the gridsize of about $9 \mathrm{~m}$ (dimension of the spline space: 22,399) with parameters $\kappa=0.5, M_{\min }=300, M_{\max }=100$, starting from degree 1 at the local stage. The spline surface is presented in Fig. 17b. (We multiply the spline by the factor 4 in order to emphasize the details.) The computational time is 23.8 seconds. The main purpose of this first run is to remove outliers and reduce noise present in the data due to measurement errors. This is achieved by leaving out all points $(12,980$ or $2 \%)$ whose height difference to the spline surface exceeds the rms error $(0.6096 \mathrm{~m})$ of our approximation. The cleaned dataset consists of 621,624 points with heights between $-25.09 \mathrm{~m}$ and $-6.42 \mathrm{~m}$. Then we choose the $C^{1}$ spline space with gridsize $\approx$ $3.6 \mathrm{~m}$ and dimension 142,027, and apply the averaged method $Q_{1}^{a v}$ with parameters $\kappa=5, M_{\min }=3, M_{\max }=49$ to the cleaned data. The computational time for this second run is 114.6 seconds, and the resulting surface is presented in Fig. 17c and $17 \mathrm{~d}$. It faithfully represents a fine structure on the harbour floor, in particular, remains from the dredge process. In addition, Fig. 18 shows the robust behavior of the spline in the presence of outliers and demonstrates the effects of cleaning. Errors (in meters) with respect to the full data set as well as to the subset of "clean" data obtained as explained above are presented in Tab. 6. (We do not provide the maximal error to the raw data since it is obviously useless.)

\begin{tabular}{|c|c|c|c|c|c|c|c|c|}
\hline & & & & \multicolumn{2}{|c|}{ raw data } & \multicolumn{3}{|c|}{ cleaned data } \\
\cline { 5 - 9 } fig & $n$ & $m$ & $D$ & mean & rms & max & mean & rms \\
\hline $17 \mathrm{~b}$ & 40 & 109 & 22,399 & 0.1821 & 0.6096 & 0.6096 & 0.1346 & 0.1780 \\
$17 \mathrm{c}$ & 100 & 281 & 142,027 & 0.0995 & 0.6016 & 1.0774 & 0.0576 & 0.0784 \\
\hline
\end{tabular}

Tab. 6. Errors in tests with Rotterdam port data. 


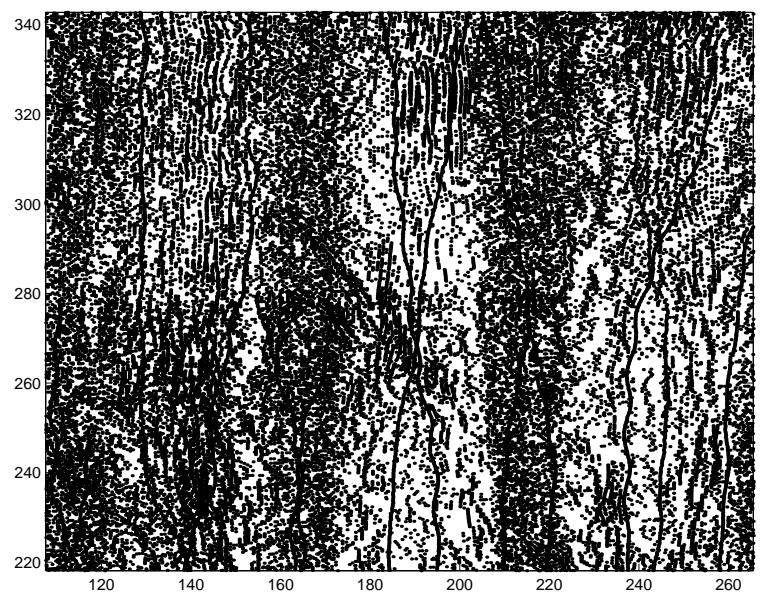

(a)

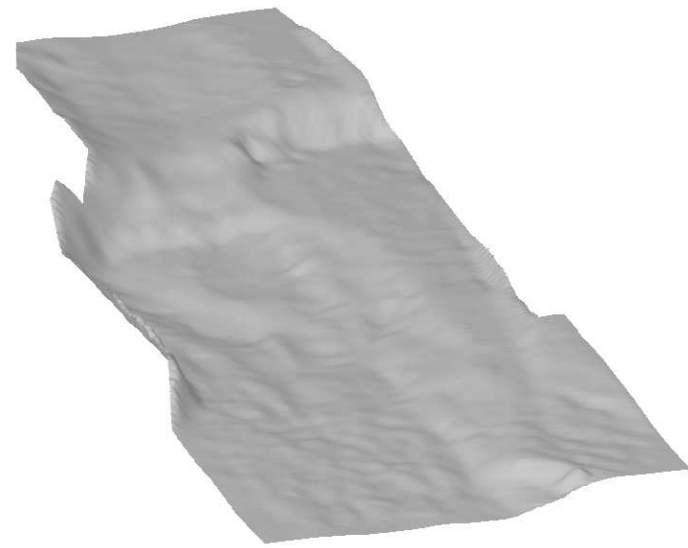

(b)

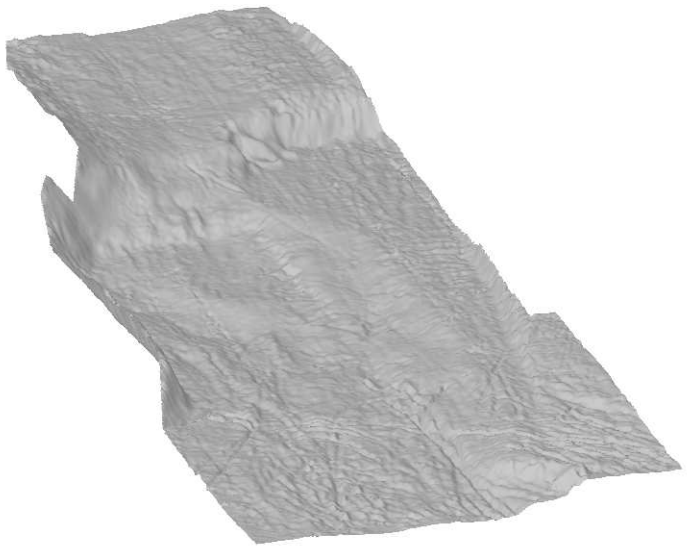

(c)

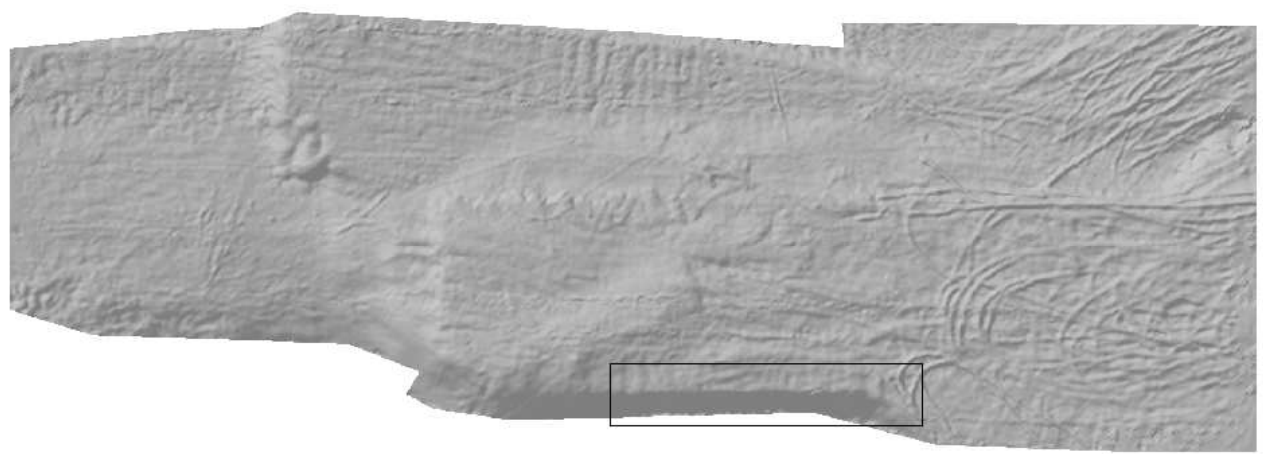

(d)

Fig. 17. $C^{1}$ spline approximation of Rotterdam port data: (a) Locations of the data points in a subregion. (b) Coarse spline approximation obtained by direct application of the operator $Q_{1}^{a v}$ to the raw data. This spline is used to clean the data. (c) Fine spline approximation $\left(Q_{1}^{a v}\right)$ to the cleaned data. (d) View from the above. 


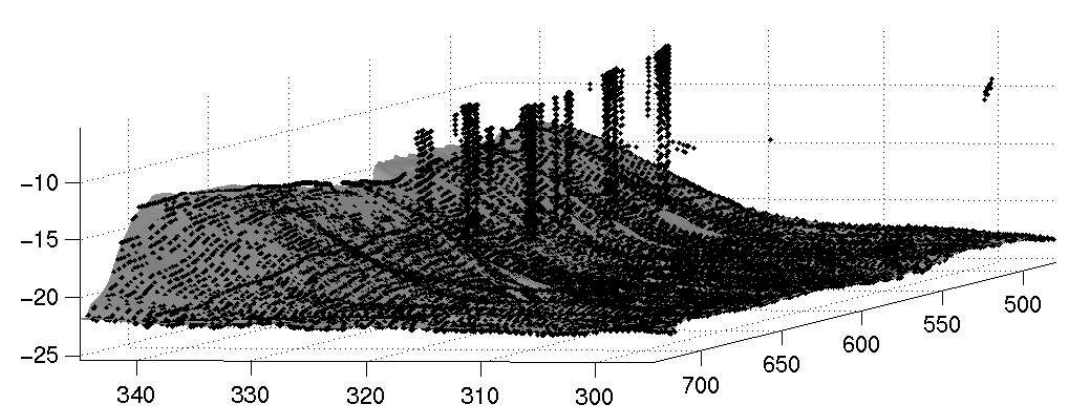

(a)

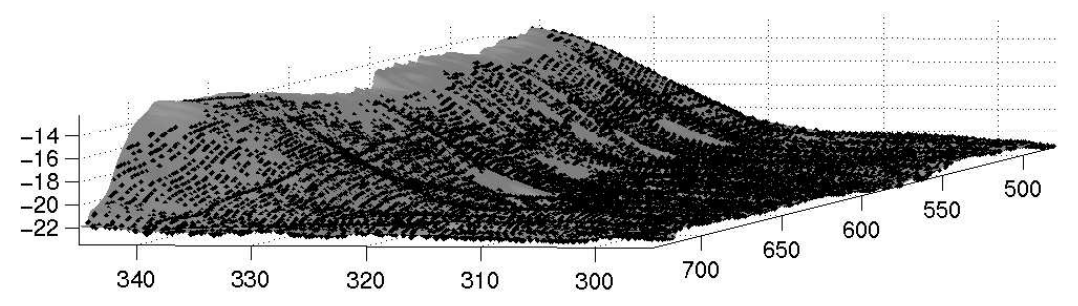

(b)

Fig. 18. Effect of cleaning of the Rotterdam port data: The $C^{1}$ spline approximation in the rectangle indicated in Fig. 17d with (a) raw data and (b) cleaned data (data reduction $2 \%$ ).

Remark 6.1. As explained above, our algorithm tries to fit polynomials of the highest possible degree in each local subregion, and drops the degree successively if the criterion (2.17) is not satisfied. In Tab. 7 we sum up the information on the degrees of local polynomials computed in the tests of Sections 6.2 and 6.4-6.6. Recall that practically all local patches are of the highest degree in the approximation order and compression tests of Sections 6.1 and 6.3, respectively.

\begin{tabular}{|c|r|r|r|r|c|c|c|}
\hline fig & \multicolumn{1}{|c|}{0} & \multicolumn{1}{c|}{1} & \multicolumn{1}{c|}{2} & \multicolumn{1}{c|}{3} & 4 & 5 & 6 \\
\hline $12 \mathrm{c}$ & $1.6 \%$ & $43.8 \%$ & $48.8 \%$ & $5.9 \%$ & - & - & - \\
$12 \mathrm{~d}$ & $0.0 \%$ & $0.0 \%$ & $14.3 \%$ & $30.6 \%$ & $25.5 \%$ & $21.4 \%$ & $8.2 \%$ \\
$14 \mathrm{~b}$ & $16.0 \%$ & $23.5 \%$ & $0.0 \%$ & $60.5 \%$ & - & - & - \\
$14 \mathrm{~d}$ & $45.7 \%$ & $17.6 \%$ & $19.1 \%$ & $17.6 \%$ & - & - & - \\
15 & $1.7 \%$ & $16.7 \%$ & $7.8 \%$ & $12.8 \%$ & $22.3 \%$ & $38.8 \%$ & - \\
16 & $1.7 \%$ & $38.4 \%$ & $30.9 \%$ & $21.7 \%$ & $6.7 \%$ & $0.6 \%$ & - \\
$17 \mathrm{~b}$ & $26.3 \%$ & $73.7 \%$ & - & - & - & - & - \\
$17 \mathrm{c}$ & $17.1 \%$ & $4.6 \%$ & $1.9 \%$ & $76.3 \%$ & - & - & - \\
\hline
\end{tabular}

Tab. 7. Degrees of local polynomials: The columns numbered $0-6$ contain the percentage of the polynomials of corresponding degrees for the surfaces shown in the figures indicated in the first column.

Remark 6.2. We note that the above computational times can often be significantly reduced if one pursues the goal of obtaining approximations of sufficient 
quality for practice rather than the best possible quality. For example, the nonaveraged splines are computed 8 times faster. In addition, bigger $\kappa$ and smaller $M_{\min }, M_{\max }$ also reduce costs. Pivoted QR decomposition can be used for the estimation of $\sigma_{\min }$ instead of the more reliable (but, in general, also more expensive) singular value decomposition employed in this paper. Finally, without any loss of quality, an easily implementable parallel version of the first (local) stage of the algorithm should reduce overall computational times by a factor nearing the number of processors on a multiprocessor machine.

Remark 6.3. The usability and efficiency of the non-averaged $C^{1}$ method in the context of interactive visualization and rendering of large terrain data has been demonstrated in [23], where real-time frame rates for typical fly-through sequences are achieved.

Acknowledgement. We thank David Stelpstra from the company QPS (Quality Positioning Services), Zeist, The Netherlands, for providing us with the Rotterdam port data set, which was recorded using the QINSy software.

\section{References}

1. Alfeld, P. and L. L. Schumaker, The dimension of bivariate spline spaces of smoothness $r$ for degree $d \geq 4 r+1$, Constr. Approx. 3 (1987), 189-197.

2. Alfeld, P. and L. L. Schumaker, Smooth macro-elements based on CloughTocher triangle splits, Numer. Math. 90 (2002), 597-616.

3. Alfeld, P. and L. L. Schumaker, Smooth macro-elements based on Powell-Sabin triangle splits, Advances in Comp. Math. 16 (2002), 29-46.

4. Björck, Å., Numerical Methods for Least Squares Problems, SIAM, Philadelphia, 1996.

5. Boor, C. de, B-form basics, in Geometric Modeling: Algorithms and New Trends, G. E. Farin (ed), SIAM Publications, Philadelphia, 1987, 131-148.

6. Chui, C. K., Multivariate Splines, CBMS-NSF Reg. Conf. Series in Appl. Math., vol. 54, SIAM, Philadelphia, 1988.

7. Chui, C. K., D. Hong, and R.-Q. Jia, Stability of optimal order approximation by bivariate splines over arbitrary triangulations, Trans. Amer. Math. Soc. 347 (1995), 3301-3318.

8. Clough, R. W. and J. L. Tocher, Finite element stiffness matries for analysis of plates in bending, in Proc. Conf. on Matrix Methods in Structural Mechanics, Wright Patterson A.F.B., Ohio, 1965.

9. Davydov, O., On the approximation power of local least squares polynomials, in Algorithms for Approximation IV, J. Levesley, I. J. Anderson and J. C. Mason (eds.), University of Huddersfield, UK, 2002, 346-353.

10. Davydov, O. and G. Nürnberger, Interpolation by $C^{1}$ splines of degree $q \geq 4$ on triangulations, J. Comput. Appl. Math. 126 (2000), 159-183. 
11. Davydov, O., G. Nürnberger, and F. Zeilfelder, Interpolation by Splines on Triangulations, in New Developments in Approximation Theory, M. W. Müller, Buhmann, M. D., Mache, D. H., and Felten, M. (eds.), Birkhäuser, 1999, 49-70.

12. Davydov, O., G. Nürnberger, and F. Zeilfelder, Cubic spline interpolation on nested polygon triangulations, in Curve and Surface Fitting, A. Cohen, C. Rabut, and L. L. Schumaker (eds.), Vanderbilt University Press, Nashville, 2000, 161-170.

13. Davydov, O., G. Nürnberger, and F. Zeilfelder, Bivariate spline interpolation with optimal approximation order, Constr. Approx. 17 (2001), 181-208.

14. Davydov, O. and L. L. Schumaker, On stable local bases for bivariate polynomial spline spaces, Constr. Approx. 18 (2002), 87-116.

15. Farin, G., Triangular Bernstein-Bézier patches, Comput. Aided Geom. Design 3 (1986), 83-127.

16. Farin, G., Curves and Surfaces for Computer Aided Geometric Design, Academic Press, San Diego, 1993.

17. Foley, T. A., Scattered data interpolation and approximation with error bounds, Comput. Aided Geom. Design 3 (1986), 163-177.

18. Fraeijs de Veubeke, B., A conforming finite element for plate bending, J. Solids Structures 4 (1968), 95-108.

19. Franke, R., A critical comparison of some methods for interpolation of scattered data, Report NPS-53-79-003, Naval Postgraduate School, 1979.

20. Franke, R., Scattered data interpolation: Test of some methods, Math. Comp. 38 (1982), 181-200.

21. Franke, R., Homepage, http://www.math.nps.navy.mil/ rfranke/, Naval Postgraduate School.

22. Franke, R. and H. Hagen, Least squares surface approximation using multiquadrics and parametric domain distortion, Comput. Aided Geom. Design 16 (1999), 177-196.

23. Haber, J., F. Zeilfelder, O. Davydov, and H.-P. Seidel, Smooth approximation and rendering of large scattered data sets, in Proceedings of IEEE Visualisation 2001, T. Ertl, Joy K., and Varshney A. (eds.), 2001, 341-347, 571.

24. Laghchim-Lahlou, M. and P. Sablonnière, Triangular finite elements of HCT type and class $C^{\rho}$, Advances in Comp. Math. 2 (1994), 101-122.

25. Laghchim-Lahlou, M. and P. Sablonnière, Quadrilateral finite elements of FVS type and class $C^{\rho}$, Numer. Math. 70 (1995), 229-243.

26. Laghchim-Lahlou, M. and P. Sablonnière, $C^{r}$-finite elements of Powell-Sabin type on the three directional mesh, Advances in Comp. Math. 6 (1996), 191206.

27. Lai, M.-J., Approximation order from bivariate $C^{1}$ cubics on a four-direction mesh is full, Comput. Aided Geom. Design 11 (1994), 215-223. 
28. Lai, M.-J., Bivariate spline spaces on FVS-triangulations, in Approximation Theory VIII, Vol.1: Approximation and Interpolation, C. K. Chui and L. L. Schumaker (eds.), World Scientific Publishing Co., Inc., Singapore, 1995, 309316.

29. Lai, M.-J., Scattered data interpolation and approximation by $C^{1}$ piecewise cubic polynomials, Comput. Aided Geom. Design 13 (1996), 81-88.

30. Lai, M.-J. and L. L. Schumaker, Scattered data interpolation using $C^{2}$ supersplines of degree six, SIAM J. Numer. Anal. 34 (1997), 905-921.

31. Lai, M.-J. and L. L. Schumaker, On the approximation power of bivariate splines, Advances in Comp. Math. 9 (1998), 251-279.

32. Lai, M.-J. and L. L. Schumaker, On the approximation power of splines on triangulated quadrangulations, SIAM J. Numer. Anal. 36(1) (1999), 143-159.

33. Lai, M.-J. and L. L. Schumaker, Quadrilateral macro-elements, SIAM J. Math. Anal. 33 (2002), 1107-1116.

34. Lancaster, P. and K. Šalkauskas, Curve and Surface Fitting, Academic Press, San Diego, 1986.

35. Lavery, J. E., Shape-preserving, multiscale interpolation by bi- and multivariate cubic $L_{1}$ splines, Comput. Aided Geom. Design 18 (2001), 321-343.

36. Lodha, S. K. and R. Franke, Scattered data techniques for surfaces, in Proceedings of Dagstuhl Conference on Scientific Visualization, H. Hagen, G. Nielson and F. Post (eds.), IEEE Computer Society Press, 1999, 182-222.

37. Morandi Cecchi, M., S. De Marchi, and D. Fasoli, A package for representing $\left(C^{1}\right)$ interpolating surfaces: application to the lagoon of Venice's bed, Numer. Algorithms 20 (2,3) (1999), 197-215.

38. McMahon, J. R. and R. Franke, Knot selection for least squares thin plate splines, SIAM J. Sci. Stat. Comput. 13 (1992), 484-498.

39. Morandi, R. and A. Sestini, Data reduction in surface approximation, in Mathematical Methods for Curves and Surfaces III: Oslo 2000, T. Lyche and L. L. Schumaker (eds.), Vanderbilt University Press, 2001, 315-324.

40. Nielson, G. M., A first-order blending method for triangles based upon cubic interpolation, Internat. J. Numer. Meth. Engr. 15 (1978), 308-318.

41. Nürnberger, G., Approximation order of bivariate spline interpolation, J. Approx. Theory 87 (1996), 117-136.

42. Nürnberger, G. and Th. Rießinger, Lagrange and Hermite interpolation by bivariate splines, Numer. Func. Anal. Optim. 13 (1992), 75-96.

43. Nürnberger, G. and Th. Rießinger, Bivariate spline interpolation at grid points, Numer. Math. 71 (1995), 91-119.

44. Nürnberger, G., L. L. Schumaker, and F. Zeilfelder, Local Lagrange interpolation by bivariate $C^{1}$ cubic splines, in Mathematical Methods for Curves and 
Surfaces III: Oslo 2000, T. Lyche and L. L. Schumaker (eds.), Vanderbilt University Press, 2001, 393-404.

45. Nürnberger, G., L. L. Schumaker, and F. Zeilfelder, Lagrange interpolation by $C^{1}$ cubic splines on triangulations of separable quadrangulations, in Approximation Theory X: Splines, Wavelets, and Applications, C.K. Chui, T. Lyche, and L. L. Schumaker (eds.), Vanderbilt University Press, 2002, 405-424.

46. Nürnberger, G., L. L. Schumaker, and F. Zeilfelder, Lagrange interpolation by $C^{1}$ cubic splines on triangulated quadrangulations, to appear in Advances in Comp. Math., 2002.

47. Nürnberger, G. and G. Walz, Error analysis in interpolation by bivariate $C^{1}$ splines, IMA J. Numer. Anal. 18 (1998), 485-508.

48. Nürnberger, G. and F. Zeilfelder, Interpolation by spline spaces on classes of triangulations, J. Comput. Appl. Math. 119 (2000), 347-376.

49. Nürnberger, G. and F. Zeilfelder, Developments in bivariate spline interpolation, J. Comput. Appl. Math. 121 (2000), 125-152.

50. Nürnberger, G. and F. Zeilfelder, Local Lagrange interpolation by cubic splines on a class of triangulations, in Proceedings Conference Trends in Approximation Theory, K. Kopotun, T. Lyche, and M. Neamtu (eds.), Vanderbilt University Press, 2000, 341-350.

51. Nürnberger, G. and F. Zeilfelder, Local Lagrange interpolation on PowellSabin triangulations and terrain modelling, in Recent Progress in Multivariate Approximation, W. Haußmann, K. Jetter, and M. Reimer (eds.), Birkhäuser ISNM 137, Basel, 2001, 227-244.

52. Nürnberger, G. and F. Zeilfelder, Lagrange interpolation by bivariate $C^{1}$ splines with optimal approximation order, to appear in Advances in Comp. Math., 2002.

53. Powell, M. J. D., Radial basis functions for multivariable interpolation: a review, in Algorithms for the Approximation of Functions and Data, J. C. Mason and M. G. Cox (eds.), Oxford University Press, Oxford, 1987, 143-167.

54. Powell, M. J. D. and M. A. Sabin, Piecewise quadratic approximation on triangles, ACM Trans. Math. Software 4 (1977), 316-325.

55. Sander, G., Bornes supérieures et inférieures dans l'analyse matricielle des plaques en flexion-torsion, Bull. Soc. Royale Sciences Liège 33 (1964), 456494.

56. Schaback, R., Remarks on meshless local construction of surfaces, in The Mathematics of Surfaces IX, R. Cipolla et al. (eds.), Springer, 2000, 34-58.

57. Schumaker, L. L., Fitting surfaces to scattered data, in Approximation Theory II, G. G. Lorentz, C. K. Chui, and L. L. Schumaker (eds.), Academic Press, New York, 1976, 203-268. 
58. Schumaker, L. L., Two-stage methods for fitting surfaces to scattered data, in Quantitative Approximation, R. Schaback and K. Scherer (eds.), Lecture Notes 556, Springer, Berlin, 1976, 378-389.

59. Schumaker, L. L., Bounds on the dimension of spaces of multivariate piecewise polynomials, Rocky Mountain J. Math. 14 (1984), 251-264.

60. Wendland, H., Local polynomial reproduction and moving least squares approximation, IMA J. Numer. Anal. 21 (2001), 285-300. 